\title{
Ruthenium(0)-Catalyzed C-H Arylation of Aromatic Imines under Neutral Conditions: Access to Biaryl Aldehydes
}

\author{
Feng $\mathrm{Hu}^{\dagger}$ and Michal Szostak*, ${ }^{\dagger}$ \\ 'Department of Chemistry, Rutgers University, 73 Warren Street, Newark, New Jersey 07102, \\ United States \\ michal.szostak@rutgers.edu
}

Supporting Information

Table of Contents $\quad 1$

Additional References $\quad 2$

List of Known Compounds and General Methods $\quad 4$

Experimental Procedures and Characterization Data 5

- General Procedures 5

- $\mathrm{Ru}(0)$-Catalyzed C-H Arylation: Variation of Organoborane 7

- $\mathrm{Ru}(0)$-Catalyzed C-H Arylation: Variation of Imine 17

- $\mathrm{Ru}(0)$-Catalyzed Formal Arylation of Aldehydes via in situ Imine Synthesis 26

- Mechanistic Selectivity Studies 27

- Product Transformations 29

References

${ }^{1} \mathrm{H}$ and ${ }^{13} \mathrm{C}$ NMR Spectra

Corresponding Author:

Prof. Dr. M. Szostak

Department of Chemistry, Rutgers University

73 Warren Street, Newark, NJ 07102, United States

E-mail: michal.szostak@ rutgers.edu 


\section{Additional References}

(1) $\mathrm{Ru}(0)$-catalyzed $\mathrm{C}-\mathrm{H}$ activation of imines:

(a) Kakiuchi, F.; Yamauchi, M.; Chatani, N.; Murai, S. Chem. Lett. 1996, 111.

(b) Kakiuchi, F.; Sato, T.; Tsujimoto, T.; Yamauchi, M.; Chatani, N.; Murai, S. Chem. Lett. 1998, 1053.

(2) $\mathrm{Ru}(0)$-catalyzed $\mathrm{C}-\mathrm{H}$ activation with boronates:

(a) Hiroshima, S.; Matsumura, D.; Kochi, T.; Kakiuchi, F. Org. Lett. 2010, 12, 5318.

(b) Ueno, S.; Chatani, N.; Kakiuchi, F. J. Org. Chem. 2007, 72, 3600.

(c) Pastine, S. J.; Gribkov, D. V. Sames, D. J. Am. Chem. Soc. 2006, 128, 14220.

(d) Schwartz, M.; Dastbaravardeh, N.; Kirchner, M.; Schnürch, M.; Mihovilovic, M. D. Monatsh. Chem. 2013, 133, 539.

(e) Dastbaravardeh, N.; Schnürch, M.; Mihovilovic, M. D. Org. Lett. 2012, 14, 1930.

(f) Dastbaravardeh, N.; Kirchner, M.; Schnürch, M.; Mihovilovic, M. D. J. Org. Chem. 2013, 78, 658.

(3) $\mathrm{Ru}(\mathrm{II})$-catalyzed arylation of imines:

(a) Oi, S.; Ogino, Y.; Fukita, S.; Inoue, Y. Org. Lett. 2002, 4, 1783.

(b) Li, B.; Darcel, C.; Roisnel, T.; Dixneuf, P. H. J. Organomet. Chem. 2015, 793, 200.

(c) Li, B.; Bheeter, C. B.; Darcel, C.; Dixneuf, P. H. ACS Catal. 2011, 1, 1221.

(d) Li, B.; Devaraj, K.; Darcel, C.; Dixneuf, P. H. Tetrahedron 2012, 68, 5179.

(4) $\mathrm{Ru}(\mathrm{II})$-catalyzed alkenylation of aldehydes:

(a) Padala, K.; Jeganmohan, M. Org. Lett. 2012, 14, 1134.

(5) $\mathrm{Ru}(\mathrm{II})$-catalyzed arylation using boronic acids:

(a) Sollert, C.; Devaraj, K.; Orthaber, A.; Gates, P. J.; Pilarski, L. T. Chem. Eur. J. 2015, 21, 5380.

(b) Li, H.; Wei, W.; Xu, Y.; Zhang, C.; Wan, X. Chem. Commun. 2011, 47, 1497. 
(c) Chinnagolla, R. K.; Jeganmohan, M. Org. Lett. 2012, 14, 5246.

(d) Chinnagolla, R. K.; Jeganmohan, M. Chem. Commun. 2014, 50, 2442.

- See also:

(e) Yamamoto, T.; Yamakawa, T. RSC Adv. 2015, 5, 105829. 


\section{List of Known Compounds and General Methods}

All starting materials reported in the manuscript have been previously described in literature or are commercially available. Imines were prepared by standard methods. ${ }^{1-5}$ Organoboranes were prepared by standard methods. ${ }^{6,7}$ All experiments involving ruthenium were performed using Schlenk or glovebox techniques under argon or nitrogen atmosphere unless stated otherwise. All solvents were purchased at the highest commercial grade and used as received or after purification by passing through activated alumina columns or distillation from sodium/benzophenone under nitrogen. All solvents were deoxygenated prior to use. All other chemicals were purchased at the highest commercial grade and used as received. Reaction glassware was oven-dried at $140{ }^{\circ} \mathrm{C}$ for at least $24 \mathrm{~h}$ or flame-dried prior to use, allowed to cool under vacuum and purged with argon (three cycles). All products were identified using ${ }^{1} \mathrm{H}$ NMR and GC-MS analysis and comparison with authentic samples. GC and/or GC/MS analysis was used for volatile products. ${ }^{1} \mathrm{H}$ NMR and ${ }^{13} \mathrm{C}$ NMR spectra were recorded in $\mathrm{CDCl}_{3}$ on Bruker spectrometers at $500\left({ }^{1} \mathrm{H}\right.$ NMR $)$ and $125 \mathrm{MHz}\left({ }^{13} \mathrm{C}\right.$ NMR). All shifts are reported in parts per million (ppm) relative to residual $\mathrm{CHCl}_{3}$ peak $\left(7.27\right.$ and $77.2 \mathrm{ppm},{ }^{1} \mathrm{H} \mathrm{NMR}$ and ${ }^{13} \mathrm{C} \mathrm{NMR}$, respectively). All coupling constants (J) are reported in hertz (Hz). Abbreviations are: s, singlet; d, doublet; t, triplet; q, quartet; brs, broad singlet. GC-MS chromatography was performed using Agilent HP6890 GC System and Agilent 5973A inert XL EI/CI MSD using helium as the carrier gas at a flow rate of $1 \mathrm{~mL} / \mathrm{min}$ and an initial oven temperature of $50^{\circ} \mathrm{C}$. GC-MS chromatography was performed using Agilent HP6890 GC System and Agilent 5973A inert XL EI/CI MSD using helium as the carrier gas at a flow rate of $1 \mathrm{~mL} / \mathrm{min}$ and an initial oven temperature of $50{ }^{\circ} \mathrm{C}$. The injector temperature was $250{ }^{\circ} \mathrm{C}$. The detector temperature was $250{ }^{\circ} \mathrm{C}$. For runs with the initial oven temperature of $50{ }^{\circ} \mathrm{C}$, temperature was increased with a $10{ }^{\circ} \mathrm{C} / \mathrm{min}$ ramp after $50{ }^{\circ} \mathrm{C}$ hold for $3 \mathrm{~min}$ to a final temperature of $280{ }^{\circ} \mathrm{C}$, then hold at $280{ }^{\circ} \mathrm{C}$ for $30 \mathrm{~min}$ (splitless mode of injection, total run time of $53.00 \mathrm{~min}$ ). High-resolution mass spectra (HRMS) were measured on a 7T Bruker Daltonics FT-MS instrument. All flash chromatography was performed using silica gel, $60 \AA$, 300 mesh. TLC analysis was carried out on glass plates coated with silica gel 60 F254, $0.2 \mathrm{~mm}$ thickness. The plates were visualized using a $254 \mathrm{~nm}$ ultraviolet lamp or aqueous potassium permanganate solutions. ${ }^{1} \mathrm{H}$ NMR, ${ }^{13} \mathrm{C}$ NMR, MS and HRMS data are given for all compounds in the Supporting Experimental for characterization purposes. ${ }^{1} \mathrm{H}$ NMR, ${ }^{13} \mathrm{C}$ NMR, MS and HRMS data are reported for all new compounds. 


\section{Experimental Procedures and Characterization Data}

General Procedure A. Ru(0)-Catalyzed C-H Arylation. An oven-dried vial equipped with a stir bar was charged with an imine substrate (typically, $0.2 \mathrm{mmol}, 1.0$ equiv), boronic ester (typically, 1.5 equiv), $\mathrm{H}$-acceptor (typically, 1.2 equiv), $\mathrm{Ru}_{3}(\mathrm{CO})_{12}$ (typically, $5 \mathrm{~mol} \%$ ), placed under a positive pressure of argon, and subjected to three evacuation/backfilling cycles under high vacuum. Toluene (typically, $1.0 \mathrm{M}$ ) was added with vigorous stirring at room temperature, the reaction mixture was placed in a preheated oil bath at $125^{\circ} \mathrm{C}$, and stirred for an indicated time at $125{ }^{\circ} \mathrm{C}$. After the indicated time, the reaction mixture was cooled down to room temperature, diluted with $\mathrm{CH}_{2} \mathrm{Cl}_{2}(5 \mathrm{~mL})$, filtered and concentrated. The sample was analyzed by ${ }^{1} \mathrm{H} \mathrm{NMR}\left(\mathrm{CDCl}_{3}, 500 \mathrm{MHz}\right)$ and GC-MS to obtain conversion, selectivity, and yield using an internal standard and comparison with authentic samples. Unless stated otherwise, the crude product was subjected to the imine deprotection procedure using $1 \mathrm{~N}$ aqueous $\mathrm{HCl}$ in diethyl ether for $3 \mathrm{~h}$ at room temperature, and purified by chromatography on silica gel to afford analytically pure aldehyde products. Note that the imine product could typically be obtained directly by careful chromatography on silica gel or by recrystallization from methanol. ${ }^{1-5}$ Routinely, GC-MS analysis was used as a complementary method of analysis to confirm the product distribution. Unless stated otherwise, in all examples reported in the manuscript reduction/dehydrogenation selectivity is $>98: 2$.

General Procedure B. Imine Deprotection. To the solution of an imine substrate (typically, 0.2 mmol) in diethyl ether (typically, $10 \mathrm{~mL}), \mathrm{HCl}(\mathrm{aq}, 1 \mathrm{~N}, 10 \mathrm{~mL}$ ) was added. The reaction mixture was stirred for $3 \mathrm{~h}$ at room temperature. After the indicated time, the reaction mixture was diluted with diethyl ether $(10 \mathrm{~mL})$, the aqueous layer was extracted with diethyl ether $(3 \times 20$ $\mathrm{mL}$ ), organic layers were combined, dried over $\mathrm{Na}_{2} \mathrm{SO}_{4}$, filtered and concentrated. Purification by chromatography using silica gel (EtOAc/hexanes) afforded the title product.

\section{General Procedure C. Ru(0)-Catalyzed C-H Arylation of Aldehydes with an in situ Imine} Synthesis. An oven-dried vial equipped with a stir bar was charged with aryl aldehyde (typically, $0.2 \mathrm{mmol}, 1.0$ equiv), aniline (typically, $0.2 \mathrm{mmol}, 1.0$ equiv), boronic ester (typically, $0.3 \mathrm{mmol}$, 1.5 equiv), $\mathrm{H}$-acceptor (typically, 1.2 equiv), $\mathrm{Ru}_{3}(\mathrm{CO})_{12}$ (typically, $5.0 \mathrm{~mol} \%$ ), and anhydrous $\mathrm{MgSO}_{4}$ (typically, $0.3 \mathrm{mmol}, 1.5$ equiv), placed under a positive pressure of argon, and subjected 
to three evacuation/backfilling cycles under high vacuum. Toluene (typically, 1.0 M) was added with vigorous stirring at room temperature, the reaction mixture was placed in a preheated oil bath at $125{ }^{\circ} \mathrm{C}$, and stirred for at $125{ }^{\circ} \mathrm{C}$ for an indicated time. After the indicated time, the reaction mixture was cooled down to room temperature, diluted with $\mathrm{CH}_{2} \mathrm{Cl}_{2}(5 \mathrm{~mL})$, filtered and concentrated. The sample was analyzed by ${ }^{1} \mathrm{H} \mathrm{NMR}\left(\mathrm{CDCl}_{3}, 500 \mathrm{MHz}\right)$ and $\mathrm{GC}-\mathrm{MS}$ to obtain conversion, selectivity, and yield using an internal standard and comparison with authentic samples. Unless stated otherwise, the crude product was subjected to the imine deprotection procedure using $1 \mathrm{~N}$ aqueous $\mathrm{HCl}$ in diethyl ether for $3 \mathrm{~h}$ at room temperature, and purified by chromatography on silica gel to afford analytically pure aldehyde products. Note that the imine product could typically be obtained directly by careful chromatography on silica gel or by recrystallization from methanol. ${ }^{1-5}$

\section{General Procedure D. Ru(0)-Catalyzed C-H Arylation. Representative Procedure for} Large Scale Synthesis. An oven-dried vial (20 mL) equipped with a stir bar was charged with (E)- $N$-(naphthalen-2-ylmethylene)aniline (1.16 g, $5.0 \mathrm{mmol}, 1.0$ equiv), phenylboronic ester (2a, $1.43 \mathrm{~g}, 1.5$ equiv, $7.5 \mathrm{mmol}$ ), (E)-4-phenylbut-3-en-2-one (BA) (0.88 g, $6.0 \mathrm{mmol}, 1.2$ equiv), $\mathrm{Ru}_{3}(\mathrm{CO})_{12}(0.16 \mathrm{~g}, 0.25 \mathrm{mmol}, 5.0 \mathrm{~mol} \%)$, placed under a positive pressure of argon, and subjected to three evacuation/backfilling cycles under high vacuum. Toluene (1.0 M) was added with vigorous stirring at room temperature, the reaction mixture was placed in a preheated oil bath at $125^{\circ} \mathrm{C}$, and stirred at $125^{\circ} \mathrm{C}$ for $8 \mathrm{~h}$. After the indicated time, the reaction mixture was cooled down to room temperature, the reaction mixture was analyzed by ${ }^{1} \mathrm{H} \mathrm{NMR}\left(\mathrm{CDCl}_{3}, 500\right.$ $\mathrm{MHz}$ ) and GC-MS to obtain selectivity, conversion and yield using internal standard and comparison with authentic samples. Conversion >98\%. Mono-/diarylation selectivity >98:2. Purification by chromatography (EtOAc/hexanes) and recrystallization from methanol afforded the title product (3ac', $1.36 \mathrm{~g})$ as a yellow solid. Yield 88\%. Mp $=101-102{ }^{\circ} \mathrm{C} . \underline{\mathbf{1}} \mathbf{\mathrm { NMR }}(\mathbf{5 0 0}$ $\underline{\text { MHz, CDCl}} 3)^{2} \delta .41(\mathrm{~d}, J=8.6 \mathrm{~Hz}, 1 \mathrm{H}), 8.69(\mathrm{~s}, 1 \mathrm{H}), 8.00(\mathrm{~d}, J=8.4 \mathrm{~Hz}, 1 \mathrm{H}), 7.95(\mathrm{~d}, J=8.1$ $\mathrm{Hz}, 1 \mathrm{H}), 7.68$ (ddd, $J=8.5,6.8,1.4 \mathrm{~Hz}, 1 \mathrm{H}), 7.59$ (dd, $J=8.1,6.9 \mathrm{~Hz}, 1 \mathrm{H}), 7.55$ (d, $J=8.4 \mathrm{~Hz}$, $1 \mathrm{H}), 7.49$ (d, $J=3.8 \mathrm{~Hz}, 4 \mathrm{H}), 7.46-7.43$ (m, $1 \mathrm{H}), 7.42-7.38(\mathrm{~m}, 2 \mathrm{H}), 7.26-7.19(\mathrm{~m}, 3 \mathrm{H}) . \underline{{ }^{13} \mathbf{C}}$ $\underline{\text { NMR (125 MHz, CDCl}} \mathbf{3}) \delta 161.7,152.5,143.3,140.7,133.5,131.2,130.9,130.7,129.6,129.3$, $128.5,128.4,128.2,128.1,127.9,126.7,126.5,126.1,121.1$. MS $=307.1(\mathrm{EI})$. $\underline{\text { HRMS }}$ calcd for $\mathrm{C}_{23} \mathrm{H}_{17} \mathrm{NNa}\left(\mathrm{M}^{+}+\mathrm{Na}\right) 330.1259$ found 330.1261 . 


\section{Ru(0)-Catalyzed C-H Arylation: Variation of Organoborane (Scheme 1)}

3-Methyl-[1,1'-biphenyl]-2-carbaldehyde (3a, Scheme 1)

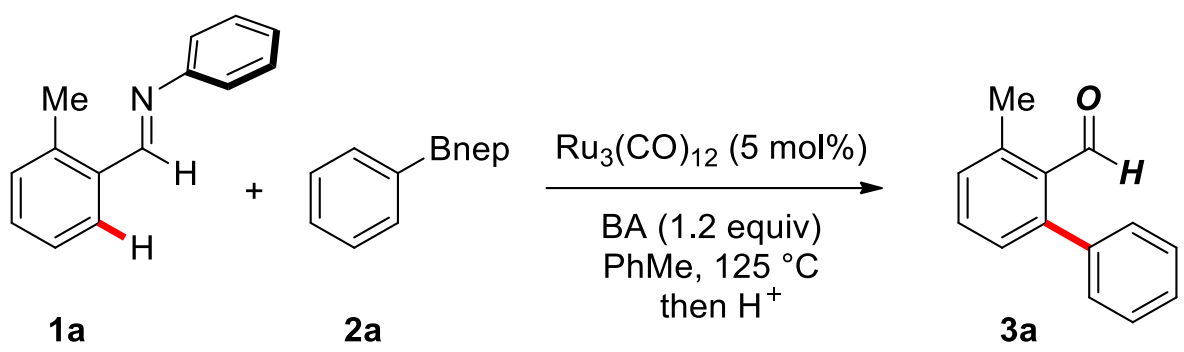

According to the general procedure A, $(E)-N$-(2-methylbenzylidene)aniline (1a, $39.1 \mathrm{mg}, 0.20$ mmol) was reacted with PhBnep (2a, $57.0 \mathrm{mg}, 0.30 \mathrm{mmol}$ ), BA (35.1 mg, $0.24 \mathrm{mmol}, 1.2$ equiv) and $\mathrm{Ru}_{3}(\mathrm{CO})_{12}(6.4 \mathrm{mg}, 5 \mathrm{~mol} \%)$ in toluene $(1.0 \mathrm{M})$ at $125{ }^{\circ} \mathrm{C}$ for $1 \mathrm{~h}$. Acidic work-up and purification by chromatography afforded 3-methyl-[1,1'-biphenyl]-2-carbaldehyde in 95\% yield

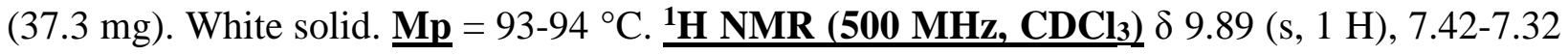

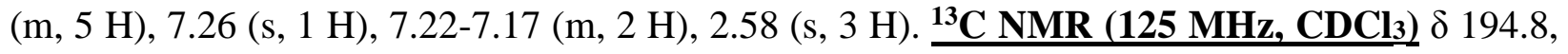
$147.3,140.2,139.1,132.8,132.3,131.3,130.2,128.8,128.5,128.1,21.7$. MS $=196.1$ (EI). $\underline{\text { HRMS }}$ calcd for $\mathrm{C}_{14} \mathrm{H}_{12} \mathrm{ONa}\left(\mathrm{M}^{+}+\mathrm{Na}\right) 219.0786$ found 219.0788 .

3,4'-Dimethyl-[1,1'-biphenyl]-2-carbaldehyde (3b, Scheme 1)
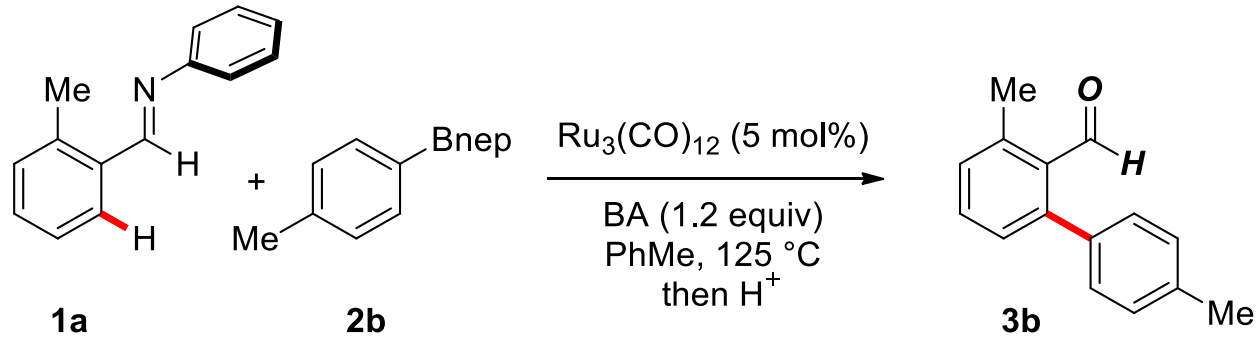

According to the general procedure A, $(E)-N$-(2-methylbenzylidene)aniline (1a, $39.1 \mathrm{mg}, 0.20$ mmol) was reacted with 4-Tol-Bnep (2b, $61.2 \mathrm{mg}, 0.30 \mathrm{mmol}), \mathrm{BA}$ (35.1 mg, $0.24 \mathrm{mmol}, 1.2$ equiv) and $\mathrm{Ru}_{3}(\mathrm{CO})_{12}(6.4 \mathrm{mg}, 5 \mathrm{~mol} \%)$ in toluene $(1.0 \mathrm{M})$ at $125{ }^{\circ} \mathrm{C}$ for $1 \mathrm{~h}$. Acidic work-up and purification by chromatography afforded 3,4'-dimethyl-[1,1'-biphenyl]-2-carbaldehyde in 94\% yield (39.5 mg). White solid. $\left.\underline{\mathbf{M p}}=90-91{ }^{\circ} \mathrm{C} . \underline{{ }^{1} \mathbf{H} \text { NMR (500 MHz, CDCl}} \mathbf{3}\right) \delta 9.97$ (s, $\left.1 \mathrm{H}\right), 7.46$ $(\mathrm{t}, J=7.4 \mathrm{~Hz}, 1 \mathrm{H}), 7.26-7.21(\mathrm{~m}, 6 \mathrm{H}), 2.65(\mathrm{~s}, 3 \mathrm{H}), 2.43(\mathrm{~s}, 3 \mathrm{H}) . \underline{{ }^{13} \mathbf{C} \text { NMR (125 MHz, }}$ $\underline{\mathbf{C D C l}_{3}} \delta \delta 194.9,147.3,140.1,138.0,136.1,132.8,132.3,131.0,130.2,129.2,128.8,21.6,21.4$. $\underline{\mathbf{M S}}=210.1(\mathrm{EI}) . \underline{\mathbf{H R M S}}$ calcd for $\mathrm{C}_{15} \mathrm{H}_{14} \mathrm{ONa}\left(\mathrm{M}^{+}+\mathrm{Na}\right) 233.0942$ found 233.0942 . 
4'-Methoxy-3-methyl-[1,1'-biphenyl]-2-carbaldehyde (3c, Scheme 1)

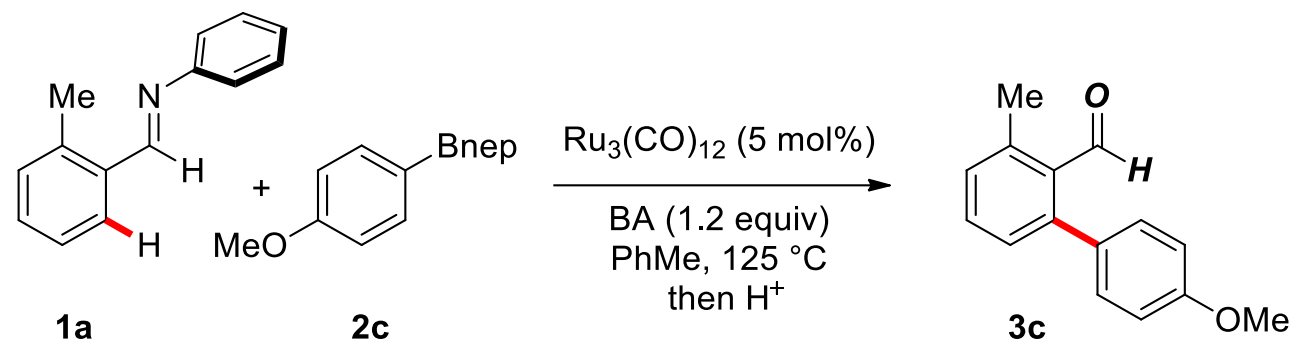

According to the general procedure A, $(E)-N$-(2-methylbenzylidene)aniline (1a, $39.1 \mathrm{mg}, 0.20$ mmol) was reacted with 4-MeO-C ${ }_{4} \mathrm{H}_{4}-\mathrm{Bnep}(2 \mathrm{c}, 66.0 \mathrm{mg}, 0.30 \mathrm{mmol})$, BA (35.0 mg, $0.24 \mathrm{mmol}$, 1.2 equiv) and $\mathrm{Ru}_{3}(\mathrm{CO})_{12}(6.4 \mathrm{mg}, 5 \mathrm{~mol} \%)$ in toluene $(1.0 \mathrm{M})$ at $125{ }^{\circ} \mathrm{C}$ for $1 \mathrm{~h}$. Acidic work-up and purification by chromatography afforded 4'-methoxy-3-methyl-[1,1'-biphenyl]-2carbaldehyde in $94 \%$ yield $(39.5 \mathrm{mg})$. White solid. $\mathrm{Mp}=100-101{ }^{\circ} \mathrm{C}$. ${ }^{\mathbf{1}} \mathbf{H}$ NMR (500 MHz, $\left.\underline{\mathbf{C D C l}_{3}}\right) \delta 10.00(\mathrm{~s}, 1 \mathrm{H}), 7.48(\mathrm{t}, J=7.5 \mathrm{~Hz}, 1 \mathrm{H}), 7.35-7.28(\mathrm{~m}, 4 \mathrm{H}), 7.01(\mathrm{~d}, J=7.9 \mathrm{~Hz}, 2 \mathrm{H})$,

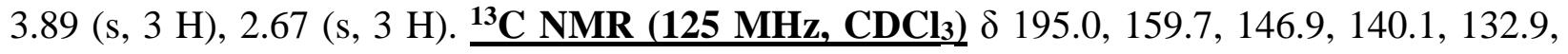
132.3, 131.4, 131.3, 130.8, 128.8, 114.0, 55.6, 21.6. $\underline{\mathbf{M S}}=226.1$ (EI). $\underline{\text { HRMS }}$ calcd for $\mathrm{C}_{15} \mathrm{H}_{14} \mathrm{O}_{2} \mathrm{Na}\left(\mathrm{M}^{+}+\mathrm{Na}\right) 249.0891$ found 249.0891.

3-Methyl-4'-(trifluoromethyl)-[1,1'-biphenyl]-2-carbaldehyde (3d, Scheme 1)
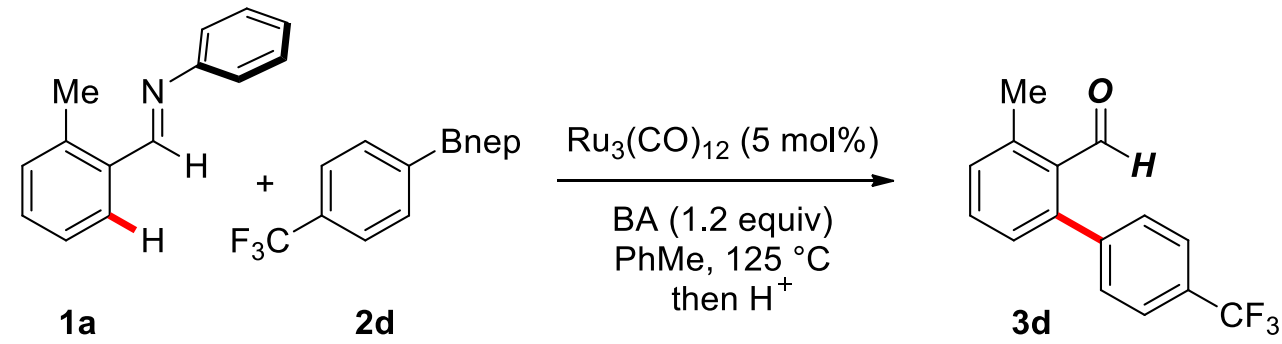

According to the general procedure A, $(E)-N$-(2-methylbenzylidene)aniline (1a, $39.1 \mathrm{mg}, 0.20$ mmol) was reacted with 4- $\mathrm{CF}_{3}-\mathrm{C}_{6} \mathrm{H}_{4}-\mathrm{Bnep}(\mathbf{2 d}, 77.4 \mathrm{mg}, 0.30 \mathrm{mmol})$, BA (35.1 mg, $0.24 \mathrm{mmol}$, 1.2 equiv) and $\mathrm{Ru}_{3}(\mathrm{CO})_{12}(6.4 \mathrm{mg}, 5 \mathrm{~mol} \%)$ in toluene $(1.0 \mathrm{M})$ at $125{ }^{\circ} \mathrm{C}$ for $1 \mathrm{~h}$. Acidic work-up and purification by chromatography afforded 3-methyl-4'-(trifluoromethyl)-[1,1'-biphenyl]-2carbaldehyde in $90 \%$ yield $(47.6 \mathrm{mg})$. White solid. $\underline{\mathbf{M p}}=82-83{ }^{\circ} \mathrm{C}$. $\underline{\mathbf{H} ~ \mathbf{~ N M R}(\mathbf{5 0 0} \mathbf{~ M H z}}$ $\underline{\left.\text { CDCl}_{3}\right)} \delta 9.99(\mathrm{~s}, 1 \mathrm{H}), 7.73(\mathrm{~d}, J=7.9 \mathrm{~Hz}, 2 \mathrm{H}), 7.55-7.50(\mathrm{~m}, 1 \mathrm{H}), 7.49$ (d, J = $\left.7.9 \mathrm{~Hz}, 2 \mathrm{H}\right)$, $7.35(\mathrm{~d}, J=7.5 \mathrm{~Hz}, 1 \mathrm{H}), 7.27(\mathrm{~d}, J=10.9 \mathrm{~Hz}, 1 \mathrm{H}), 2.69$ (s, $\left.3 \mathrm{H}) . \underline{{ }^{13} \mathbf{C ~ N M R}(125 \mathrm{MHz}, \mathbf{C D C l}} \mathbf{3}\right)$ $\delta 193.9,145.5,143.0,140.6,132.7,132.6,132.0,130.5,130.3(\mathrm{q}, J=31.2 \mathrm{~Hz}), 128.7,125.5$ (q, 


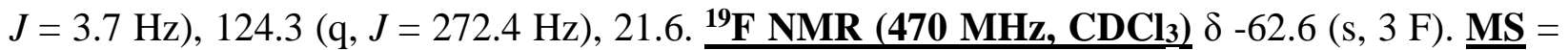
264.1 (EI). $\underline{\text { HRMS }}$ calcd for $\mathrm{C}_{15} \mathrm{H}_{11} \mathrm{~F}_{3} \mathrm{ONa}\left(\mathrm{M}^{+}+\mathrm{Na}\right) 287.0660$ found 287.0663 .

4'-Fluoro-3-methyl-[1,1'-biphenyl]-2-carbaldehyde (3e, Scheme 1)
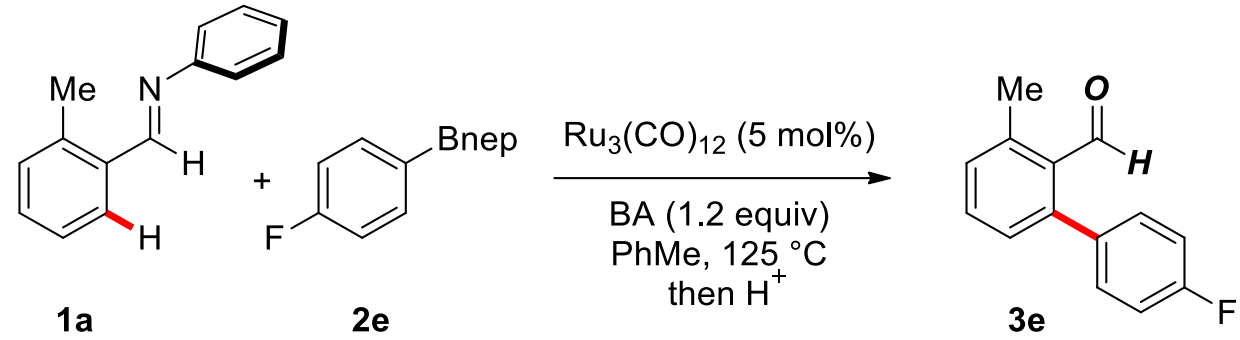

According to the general procedure A, $(E)-N$-(2-methylbenzylidene)aniline (1a, $39.1 \mathrm{mg}, 0.20$ mmol) was reacted with 4-F-C $6 \mathrm{H}_{4}$-Bnep (2e, $\left.62.4 \mathrm{mg}, 0.30 \mathrm{mmol}\right)$, BA (35.1 mg, $0.24 \mathrm{mmol}, 1.2$ equiv) and $\mathrm{Ru}_{3}(\mathrm{CO})_{12}(6.4 \mathrm{mg}, 5 \mathrm{~mol} \%)$ in toluene $(1.0 \mathrm{M})$ at $125{ }^{\circ} \mathrm{C}$ for $8 \mathrm{~h}$. Acidic work-up and purification by chromatography afforded 4'-fluoro-3-methyl-[1,1'-biphenyl]-2-carbaldehyde in $86 \%$ yield $(36.8 \mathrm{mg})$. White solid. $\underline{\mathbf{M p}}=80-83^{\circ} \mathrm{C} . \underline{\left.{ }^{1} \mathbf{H} \text { NMR (500 MHz, CDCl }\right)} \delta 9.98(\mathrm{~s}, 1$ H), 7.48 (t, $J=7.5 \mathrm{~Hz}, 1 \mathrm{H}), 7.36-7.28(\mathrm{~m}, 3 \mathrm{H}), 7.25$ (d, $J=7.5 \mathrm{~Hz}, 1 \mathrm{H}), 7.15$ (t, $J=8.2 \mathrm{~Hz}, 2$

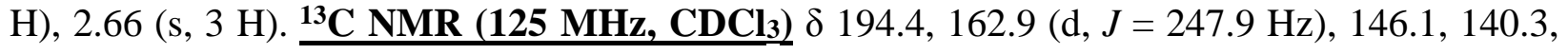
$135.1(\mathrm{~d}, J=3.4 \mathrm{~Hz}), 132.8,132.4,131.8(\mathrm{~d}, J=8.2 \mathrm{~Hz}), 131.4,128.8,115.5$ (d, $J=21.5 \mathrm{~Hz})$, 21.6. ${ }^{19} \mathbf{F}$ NMR (470 MHz, $\left.\mathbf{C D C l} 3\right) \delta-114.1$ (m, 1 F). $\underline{\text { MS }}=214.1$ (EI). $\underline{\text { HRMS }}$ calcd for $\mathrm{C}_{14} \mathrm{H}_{11} \mathrm{FONa}\left(\mathrm{M}^{+}+\mathrm{Na}\right) 237.0692$ found 237.0693 .

\section{4'-Chloro-3-methyl-[1,1'-biphenyl]-2-carbaldehyde (3f, Scheme 1)}<smiles>Cc1ccccc1/C=N/c1ccccc1</smiles>

$1 \mathbf{a}$

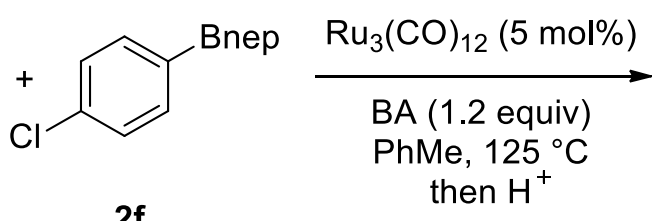

$2 f$<smiles>Cc1cccc(-c2ccc(Cl)cc2)c1C=O</smiles>

According to the general procedure A, $(E)-N$-(2-methylbenzylidene)aniline (1a, $39.1 \mathrm{mg}, 0.20$ mmol) was reacted with 4-Cl- $\mathrm{C}_{6} \mathrm{H}_{4}$-Bnep (2f, $\left.67.4 \mathrm{mg}, 0.30 \mathrm{mmol}\right)$, BA (35.1 mg, $0.24 \mathrm{mmol}$, 1.2 equiv) and $\mathrm{Ru}_{3}(\mathrm{CO})_{12}(6.4 \mathrm{mg}, 5 \mathrm{~mol} \%)$ in toluene $(1.0 \mathrm{M})$ at $125{ }^{\circ} \mathrm{C}$ for $8 \mathrm{~h}$. Acidic work-up and purification by chromatography afforded 4'-fluoro-3-methyl-[1,1'-biphenyl]-2-carbaldehyde

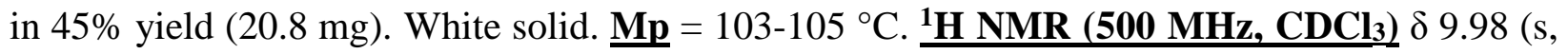


$1 \mathrm{H}), 7.48(\mathrm{t}, J=7.6 \mathrm{~Hz}, 1 \mathrm{H}), 7.43(\mathrm{~d}, J=8.1 \mathrm{~Hz}, 2 \mathrm{H}), 7.29(\mathrm{t}, J=8.4 \mathrm{~Hz}, 3 \mathrm{H}), 7.24(\mathrm{~d}, J=7.6$

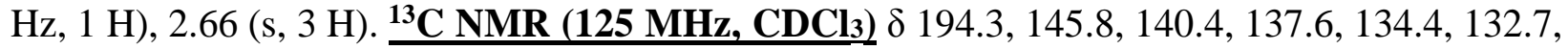
132.5, 131.6, 131.4, 128.7, 128.7, 21.6. $\underline{\mathbf{M S}}=230.0(\mathrm{EI})$. $\underline{\mathbf{H R M S}}$ calcd for $\mathrm{C}_{14} \mathrm{H}_{11} \mathrm{ClONa}\left(\mathrm{M}^{+}+\right.$ Na) 253.0396 found 253.0399.

Methyl 2'-formyl-3'-methyl-[1,1'-biphenyl]-4-carboxylate (3g, Scheme 1)

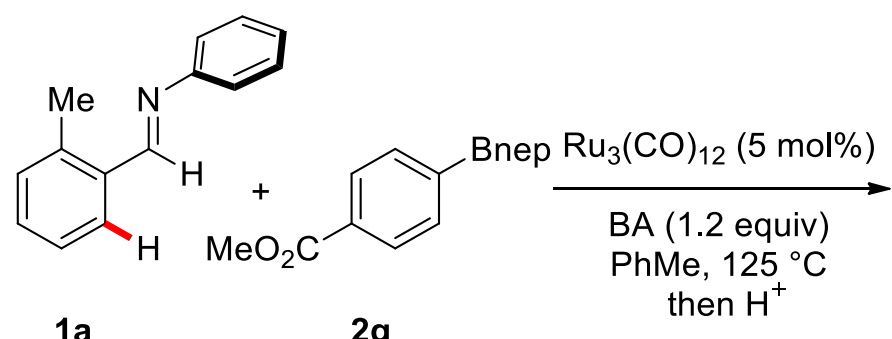

$2 \mathrm{~g}$

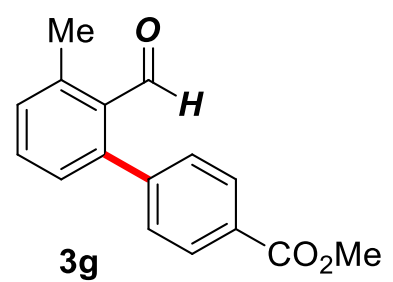

$3 g$

According to the general procedure A, $(E)-N$-(2-methylbenzylidene)aniline (1a, $39.1 \mathrm{mg}, 0.20$ mmol) was reacted with 4- $\mathrm{MeO}_{2} \mathrm{C}-\mathrm{C}_{6} \mathrm{H}_{4}-\mathrm{Bnep}(\mathbf{2 g}, 74.4 \mathrm{mg}, 0.30 \mathrm{mmol}), \mathrm{BA}(35.1 \mathrm{mg}, 0.24$ mmol, 1.2 equiv) and $\mathrm{Ru}_{3}(\mathrm{CO})_{12}(6.4 \mathrm{mg}, 5 \mathrm{~mol} \%)$ in toluene $(1.0 \mathrm{M})$ at $125{ }^{\circ} \mathrm{C}$ for $8 \mathrm{~h}$. Acidic work-up and purification by chromatography afforded methyl 2'-formyl-3'-methyl-[1,1'biphenyl]-4-carboxylate in $91 \%$ yield $(46.3 \mathrm{mg})$. White solid. $\underline{\mathbf{M p}}=91-93{ }^{\circ} \mathrm{C} . \underline{\mathbf{H}} \mathbf{\text { NMR }}(\mathbf{5 0 0}$ $\underline{\text { MHz, CDCl}} 3) \delta 9.97(\mathrm{~s}, 1 \mathrm{H}), 8.12(\mathrm{~d}, J=8.1 \mathrm{~Hz}, 2 \mathrm{H}), 7.49(\mathrm{t}, J=7.6 \mathrm{~Hz}, 1 \mathrm{H}), 7.43(\mathrm{~d}, J=8.1$ Hz, $2 \mathrm{H}), 7.32$ (d, J = 7.6 Hz, $1 \mathrm{H}), 7.26$ (d, J=7.5 Hz, $1 \mathrm{H}), 3.96$ (s, $3 \mathrm{H}), 2.67$ (s, $3 \mathrm{H}) .{ }^{13} \mathbf{C}$ $\underline{\text { NMR (125 MHz, CDCl} 3) ~} \delta 194.0,166.9,146.0,143.9,140.5,132.6,132.4,131.9,130.2,129.8$, 129.7, 128.6, 52.4, 21.6. $\underline{\mathbf{M S}}=254.1(\mathrm{EI})$. $\underline{\text { HRMS }}$ calcd for $\mathrm{C}_{16} \mathrm{H}_{14} \mathrm{O}_{3} \mathrm{Na}\left(\mathrm{M}^{+}+\mathrm{Na}\right) 277.0841$ found 277.0844 .

4'-Acetyl-3-methyl-[1,1'-biphenyl]-2-carbaldehyde (3h, Scheme 1)
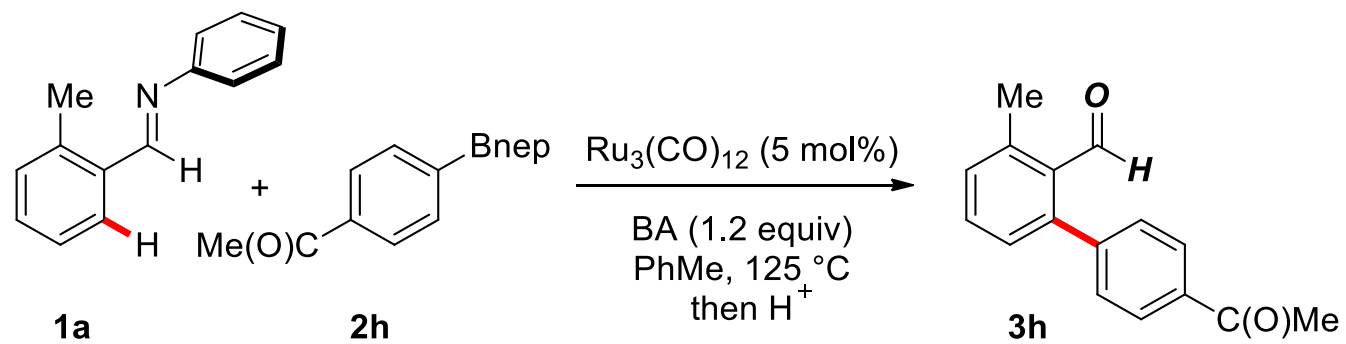

According to the general procedure A, $(E)-N$-(2-methylbenzylidene)aniline (1a, $39.1 \mathrm{mg}, 0.20$ mmol) was reacted with 4-Me(O)C-C $6 \mathrm{H}_{4}$-Bnep (2h, $\left.69.6 \mathrm{mg}, 0.30 \mathrm{mmol}\right)$, BA (35.1 mg, 0.24 mmol, 1.2 equiv) and $\mathrm{Ru}_{3}(\mathrm{CO})_{12}(6.4 \mathrm{mg}, 5 \mathrm{~mol} \%)$ in toluene $(1.0 \mathrm{M})$ at $125{ }^{\circ} \mathrm{C}$ for $8 \mathrm{~h}$. Acidic 
work-up and purification by chromatography afforded 4'-acetyl-3-methyl-[1,1'-biphenyl]-2carbaldehyde in $85 \%$ yield $(40.5 \mathrm{mg})$. White solid. $\mathrm{Mp}=121-122{ }^{\circ} \mathrm{C} . \underline{\mathbf{H}}^{\mathbf{1}} \mathbf{\mathrm { NMR }}$ (500 MHz $\underline{\left.\mathbf{C D C l}_{3}\right)} \delta 9.98(\mathrm{~s}, 1 \mathrm{H}), 8.04(\mathrm{~d}, J=8.0 \mathrm{~Hz}, 2 \mathrm{H}), 7.50(\mathrm{t}, J=7.6 \mathrm{~Hz}, 1 \mathrm{H}), 7.45(\mathrm{~d}, J=8.0 \mathrm{~Hz}, 2$ H), $7.33(\mathrm{~d}, J=7.3 \mathrm{~Hz}, 1 \mathrm{H}), 7.26(\mathrm{~d}, J=7.3 \mathrm{~Hz}, 1 \mathrm{H}), 2.67(\mathrm{~s}, 3 \mathrm{H}), 2.66(\mathrm{~s}, 3 \mathrm{H}) . \underline{{ }^{13} \mathbf{C ~ N M R}}$ $\underline{\left(125 \text { MHz, } \mathbf{C D C l}_{3}\right)} \delta 197.7,193.9,145.8,144.0,140.5,136.6,132.7$ 132.5, 131.9, 130.4, 128.5, 128.5, 26.9, 21.5. $\underline{\mathrm{MS}}=238.1(\mathrm{EI})$. $\underline{\text { HRMS }}$ calcd for $\mathrm{C}_{16} \mathrm{H}_{14} \mathrm{O}_{2} \mathrm{Na}\left(\mathrm{M}^{+}+\mathrm{Na}\right) 261.0891$ found 261.0891 .

3-Methyl-4'-vinyl-[1,1'-biphenyl]-2-carbaldehyde (3i, Scheme 1)
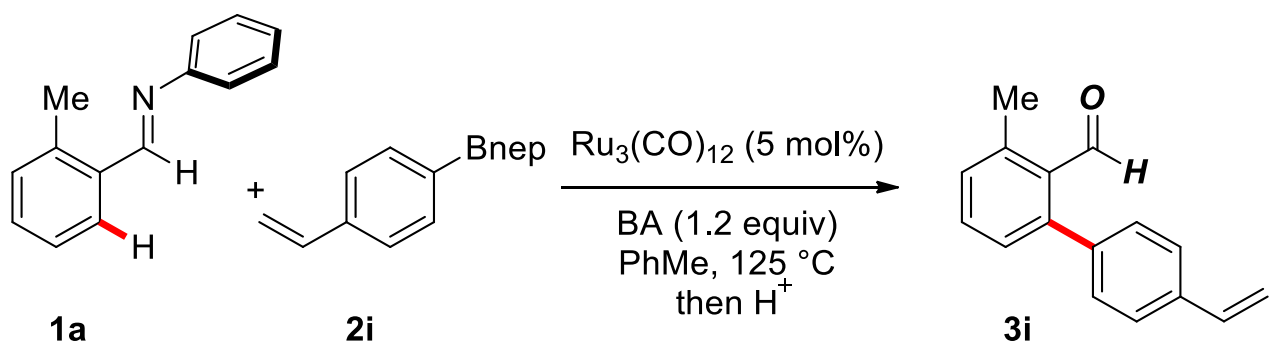

According to the general procedure A, $(E)-N$-(2-methylbenzylidene)aniline (1a, $39.1 \mathrm{mg}, 0.20$ mmol) was reacted with 4-vinyl- $\mathrm{C}_{6} \mathrm{H}_{4}-\mathrm{Bnep}(2 \mathbf{i}, 64.8 \mathrm{mg}, 0.30 \mathrm{mmol})$, BA (35.1 mg, $0.24 \mathrm{mmol}$, 1.2 equiv) and $\mathrm{Ru}_{3}(\mathrm{CO})_{12}(6.4 \mathrm{mg}, 5 \mathrm{~mol} \%)$ in toluene $(1.0 \mathrm{M})$ at $125{ }^{\circ} \mathrm{C}$ for $1 \mathrm{~h}$. Acidic work-up and purification by chromatography afforded 3-methyl-4'-vinyl-[1,1'-biphenyl]-2-carbaldehyde

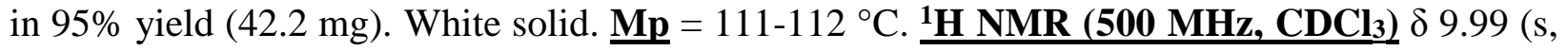
$1 \mathrm{H}), 7.49$ (d, $J=7.7 \mathrm{~Hz}, 2 \mathrm{H}), 7.46(\mathrm{~d}, J=7.7 \mathrm{~Hz}, 1 \mathrm{H}), 7.31(\mathrm{~d}, J=7.8 \mathrm{~Hz}, 2 \mathrm{H}), 7.28(\mathrm{~d}, J=$ $7.6 \mathrm{~Hz}, 2 \mathrm{H}), 6.78$ (dd, $J=17.5,10.9 \mathrm{~Hz}, 1 \mathrm{H}), 5.83$ (d, $J=17.6 \mathrm{~Hz}, 1 \mathrm{H}), 5.33$ (d, $J=10.9 \mathrm{~Hz}, 1$

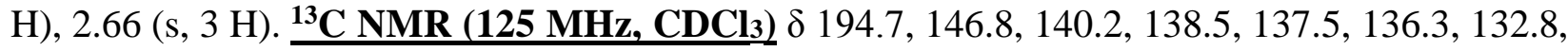
132.3, 131.3, 130.5, 128.7, 126.3, 114.9, 21.6. $\underline{\mathbf{M S}}=222.1$ (EI). $\underline{\text { HRMS }}$ calcd for $\mathrm{C}_{16} \mathrm{H}_{14} \mathrm{ONa}$ $\left(\mathrm{M}^{+}+\mathrm{Na}\right) 245.0942$ found 245.0943.

3',4'-Dimethoxy-3-methyl-[1,1'-biphenyl]-2-carbaldehyde (3j, Scheme 1) 

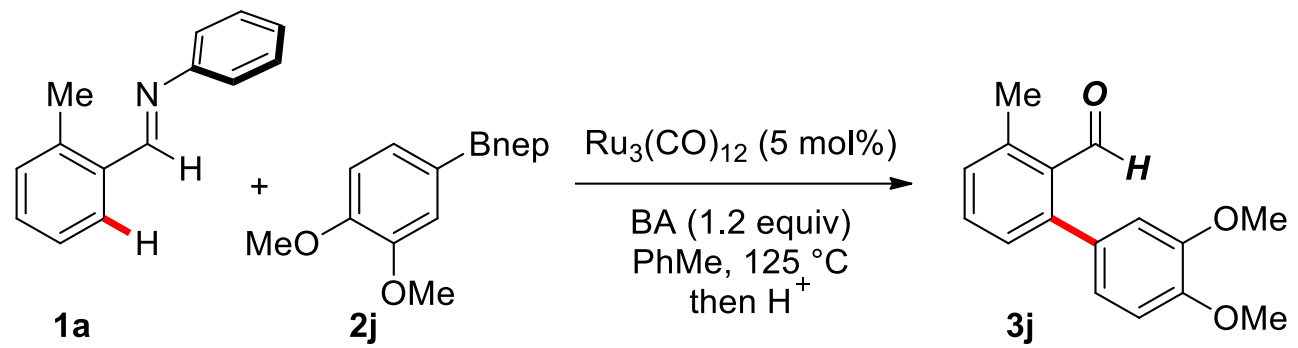

According to the general procedure A, (E)- $N$-(2-methylbenzylidene)aniline (1a, $39.1 \mathrm{mg}, 0.20$ mmol) was reacted with 3,4-(MeO) ${ }_{2}-\mathrm{C}_{6} \mathrm{H}_{4}-\mathrm{Bnep}(\mathbf{2 j}, 75.0 \mathrm{mg}, 0.30 \mathrm{mmol})$, BA (35.1 mg, 0.24 mmol, 1.2 equiv) and $\mathrm{Ru}_{3}(\mathrm{CO})_{12}(6.4 \mathrm{mg}, 5 \mathrm{~mol} \%)$ in toluene $(1.0 \mathrm{M})$ at $125{ }^{\circ} \mathrm{C}$ for $8 \mathrm{~h}$. Acidic work-up and purification by chromatography afforded 3',4'-dimethoxy-3-methyl-[1,1'-biphenyl]2-carbaldehyde in $81 \%$ yield $(41.5 \mathrm{mg})$. White solid. $\underline{\mathbf{M p}}=108-109^{\circ} \mathrm{C} . \underline{\mathbf{H}} \mathbf{\mathrm { NMR }}(\mathbf{5 0 0} \mathbf{~ M H z}$, $\underline{\left.\mathbf{C D C l}_{3}\right)} \delta 9.97(\mathrm{~s}, 1 \mathrm{H}), 7.46(\mathrm{t}, J=7.6 \mathrm{~Hz}, 1 \mathrm{H}), 7.29(\mathrm{~d}, J=7.7 \mathrm{~Hz}, 1 \mathrm{H}), 7.26(\mathrm{~d}, J=7.5 \mathrm{~Hz}, 1$ H), $6.94(\mathrm{t}, J=8.6 \mathrm{~Hz}, 1 \mathrm{H}), 6.89(\mathrm{t}, J=8.2 \mathrm{~Hz}, 1 \mathrm{H}), 6.87(\mathrm{~s}, 1 \mathrm{H}), 3.94(\mathrm{~s}, 3 \mathrm{H}), 3.90(\mathrm{~s}, 3 \mathrm{H})$, 2.65 (s, 3 H). ${ }^{13} \mathbf{C}$ NMR (125 MHz, CDCl 3 ) $\delta 194.9,149.2,148.9,146.9,140.0,133.0,132.2$, 131.7, 130.9, 128.6, 123.0, 113.3, 111.1, 56.17, 56.15, 21.6. $\underline{\mathbf{M S}}=256.1$ (EI). $\underline{\text { HRMS }}$ calcd for $\mathrm{C}_{16} \mathrm{H}_{16} \mathrm{O}_{3} \mathrm{Na}\left(\mathrm{M}^{+}+\mathrm{Na}\right) 279.0997$ found 279.0997.

3',5'-Difluoro-3-methyl-[1,1'-biphenyl]-2-carbaldehyde (3k, Scheme 1)

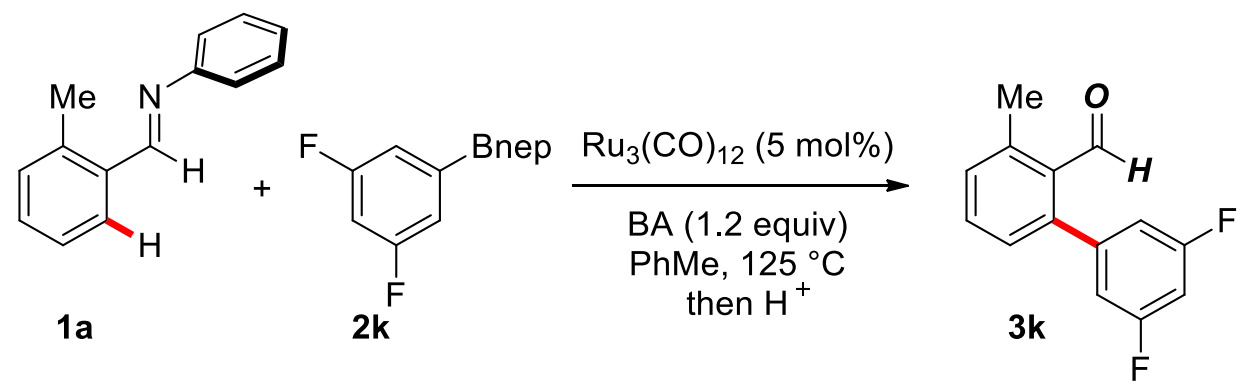

According to the general procedure A, (E)- $N$-(2-methylbenzylidene)aniline (1a, $39.1 \mathrm{mg}, 0.20$ mmol) was reacted with 3,5- $\mathrm{F}_{2}-\mathrm{C}_{6} \mathrm{H}_{4}-\mathrm{Bnep}(2 \mathbf{k}, 67.8 \mathrm{mg}, 0.30 \mathrm{mmol}), \mathrm{BA}(35.1 \mathrm{mg}, 0.24 \mathrm{mmol}$, 1.2 equiv) and $\mathrm{Ru}_{3}(\mathrm{CO})_{12}(6.4 \mathrm{mg}, 5 \mathrm{~mol} \%)$ in toluene $(1.0 \mathrm{M})$ at $125{ }^{\circ} \mathrm{C}$ for $8 \mathrm{~h}$. Acidic work-up and purification by chromatography afforded 3',5'-difluoro-3-methyl-[1,1'-biphenyl]-2carbaldehyde in $80 \%$ yield $(37.2 \mathrm{mg})$. White solid. $\mathrm{Mp}=79-80^{\circ} \mathrm{C} . \underline{{ }^{1} \mathbf{H ~ N M R}\left(\mathbf{5 0 0 ~} \mathbf{~ M H z}, \mathbf{C D C l}_{3}\right)}$ $\delta 10.02(\mathrm{~s}, 1 \mathrm{H}), 7.49$ (t, $J=7.6 \mathrm{~Hz}, 1 \mathrm{H}), 7.33(\mathrm{~d}, J=7.5 \mathrm{~Hz}, 1 \mathrm{H}), 7.23(\mathrm{~d}, J=7.5 \mathrm{~Hz}, 1 \mathrm{H})$, 6.93-6.83 (m, 3 H), 2.66 (s, 3 H). ${ }^{13} \mathbf{C}$ NMR (125 MHz, $\left.\mathbf{C D C l}_{3}\right) \delta 193.6,162.8$ (dd, $J=250.4$, $12.9 \mathrm{~Hz}), 144.6(\mathrm{t}, J=2.2 \mathrm{~Hz}), 142.54(\mathrm{t}, J=9.6 \mathrm{~Hz}), 140.7,132.6,132.2,128.4,113.3(\mathrm{dd}, J=$ 


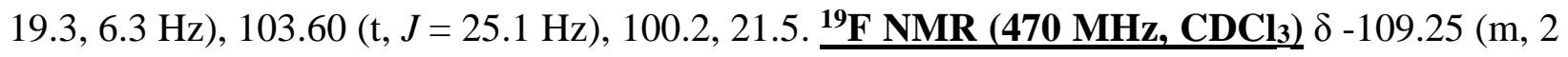
$\mathrm{F}) . \underline{\mathbf{M S}}=232.1(\mathrm{EI}) . \underline{\mathbf{H R M S}}$ calcd for $\mathrm{C}_{14} \mathrm{H}_{10} \mathrm{~F}_{2} \mathrm{ONa}\left(\mathrm{M}^{+}+\mathrm{Na}\right) 255.0597$ found 255.0598.

2-Methyl-6-(naphthalen-2-yl)benzaldehyde (31, Scheme 1)

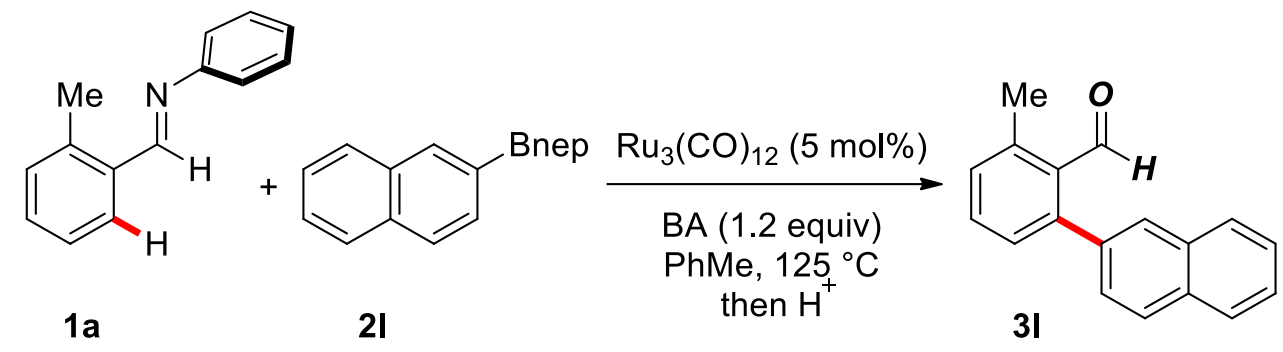

According to the general procedure A, $(E)-N$-(2-methylbenzylidene)aniline (1a, $39.1 \mathrm{mg}, 0.20$ mmol) was reacted with 2-Np-Bnep (2l, $72.0 \mathrm{mg}, 0.30 \mathrm{mmol})$, BA (35.1 mg, $0.24 \mathrm{mmol}, 1.2$ equiv) and $\mathrm{Ru}_{3}(\mathrm{CO})_{12}(6.4 \mathrm{mg}, 5 \mathrm{~mol} \%)$ in toluene $(1.0 \mathrm{M})$ at $125{ }^{\circ} \mathrm{C}$ for $1 \mathrm{~h}$. Acidic work-up and purification by chromatography afforded 2-methyl-6-(naphthalen-2-yl)benzaldehyde in $83 \%$

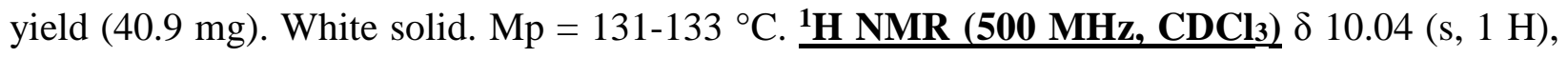
7.98-7.91 (m, 2 H), 7.91-7.85 (m, 1 H), 7.81 (s, $1 \mathrm{H}), 7.63-7.55$ (m, $2 \mathrm{H}), 7.52$ (t, J = $7.5 \mathrm{~Hz}, 2$ H), $7.39(\mathrm{~d}, J=7.5 \mathrm{~Hz}, 1 \mathrm{H}), 7.33(\mathrm{~d}, J=7.4 \mathrm{~Hz}, 1 \mathrm{H}), 2.71(\mathrm{~s}, 3 \mathrm{H}) .{ }^{{ }^{13} \mathbf{C} \text { NMR (125 MHz, }}$ $\underline{\left.\mathbf{C D C l}_{3}\right)} \delta 194.7,147.2,140.3,136.5,133.1,133.0,132.9,132.3,131.3,129.5,129.0,128.3$, 128.2, 128.0, 127.9, 126.9, 126.7, 21.9. $\underline{\mathbf{M S}}=246.1$ (EI). $\underline{\text { HRMS }}$ calcd for $\mathrm{C}_{18} \mathrm{H}_{14} \mathrm{ONa}\left(\mathrm{M}^{+}+\right.$ Na) 269.0942 found 269.0942 .

\section{2-(Benzo[d][1,3]dioxol-5-yl)-6-methylbenzaldehyde (3m, Scheme 1)}

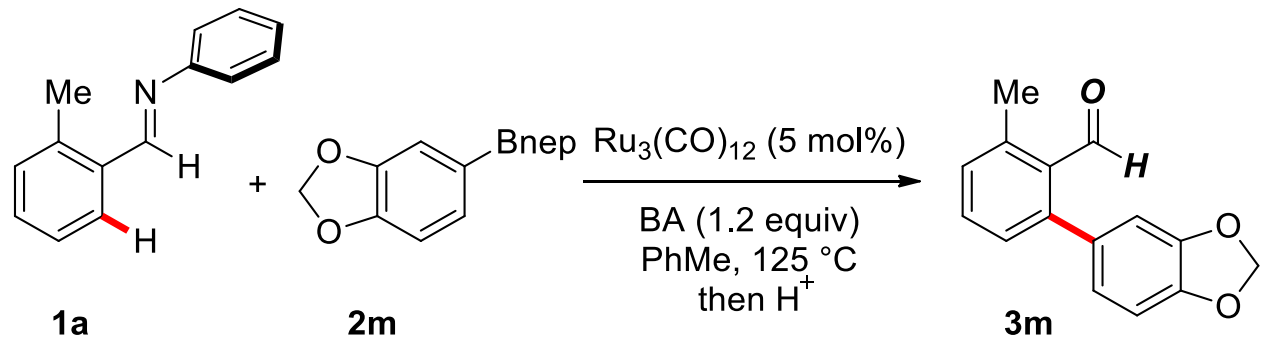

According to the general procedure A, $(E)-N$-(2-methylbenzylidene)aniline (1a, $39.1 \mathrm{mg}, 0.20$ mmol) was reacted with benzo[d][1,3]dioxol-5-yl-Bnep $(\mathbf{2 m}, 70.2 \mathrm{mg}, 0.30 \mathrm{mmol})$, BA (35.1 $\mathrm{mg}, 0.24 \mathrm{mmol}, 1.2$ equiv) and $\mathrm{Ru}_{3}(\mathrm{CO})_{12}(6.4 \mathrm{mg}, 5 \mathrm{~mol} \%)$ in toluene $(1.0 \mathrm{M})$ at $125{ }^{\circ} \mathrm{C}$ for $8 \mathrm{~h}$. Acidic work-up and purification by chromatography afforded 2-(benzo[d][1,3]dioxol-5-yl)-6methylbenzaldehyde in $88 \%$ yield $(42.3 \mathrm{mg})$. White solid. $\mathrm{Mp}=113-115^{\circ} \mathrm{C}$. ${ }^{\mathbf{1}} \mathbf{H}$ NMR (500 
$\underline{\text { MHz, CDCl}} 3) \delta 10.02(\mathrm{~s}, 1 \mathrm{H}), 7.48(\mathrm{t}, J=7.5 \mathrm{~Hz}, 1 \mathrm{H}), 7.29(\mathrm{~d}, J=6.5 \mathrm{~Hz}, 2 \mathrm{H}), 6.91(\mathrm{~d}, J=$ $7.8 \mathrm{~Hz}, 1 \mathrm{H}), 6.88(\mathrm{~s}, 1 \mathrm{H}), 6.80(\mathrm{~d}, J=7.8 \mathrm{~Hz}, 1 \mathrm{H}), 6.07(\mathrm{~s}, 2 \mathrm{H}), 2.67(\mathrm{~s}, 3 \mathrm{H}) . \underline{{ }^{13} \mathbf{C} \text { NMR (125 }}$ $\underline{\text { MHz, CDCl}} \mathbf{3}) \delta 194.7,147.9,147.8,146.7,140.1,133.0,132.9,132.3,131.1,128.6,124.3$, 110.4, 108.3, 101.6, 21.6. $\underline{\mathbf{M S}}=240.1$ (EI). $\underline{\text { HRMS }}$ calcd for $\mathrm{C}_{15} \mathrm{H}_{12} \mathrm{O}_{3} \mathrm{Na}\left(\mathrm{M}^{+}+\mathrm{Na}\right) 263.0684$ found 263.0686 .

\section{2-(Furan-3-yl)-6-methylbenzaldehyde (3n, Scheme 1)}

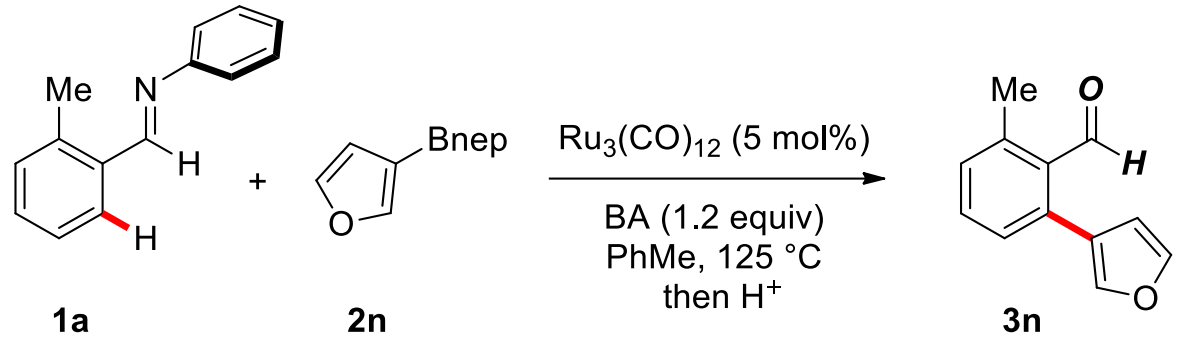

According to the general procedure A, $(E)-N$-(2-methylbenzylidene)aniline (1a, $39.1 \mathrm{mg}, 0.20$ mmol) was reacted with furan-3-yl-Bnep (2n, $54.0 \mathrm{mg}, 0.30 \mathrm{mmol})$, BA $(35.1 \mathrm{mg}, 0.24 \mathrm{mmol}$, 1.2 equiv) and $\mathrm{Ru}_{3}(\mathrm{CO})_{12}(6.4 \mathrm{mg}, 5 \mathrm{~mol} \%)$ in toluene $(1.0 \mathrm{M})$ at $125{ }^{\circ} \mathrm{C}$ for $1 \mathrm{~h}$. Acidic work-up and purification by chromatography afforded 2-(furan-3-yl)-6-methylbenzaldehyde in $60 \%$ yield (22.3 mg). Yellow oil. ${ }^{1}$ H NMR (500 MHz, CDCl 3 ) $\delta 10.23$ (s, 1 H), 7.54 (s, 1 H), 7.49 (s, 1 H), $7.46(\mathrm{t}, J=7.5 \mathrm{~Hz}, 1 \mathrm{H}), 7.30(\mathrm{~d}, J=7.6 \mathrm{~Hz}, 1 \mathrm{H}), 7.25$ (d, $J=7.8 \mathrm{~Hz}, 1 \mathrm{H}), 6.56(\mathrm{~s}, 1 \mathrm{H}), 2.66$

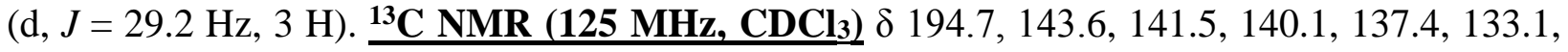
132.6, 131.3, 128.5, 123.5, 112.5, 21.5. $\underline{\mathrm{MS}}=186.1(\mathrm{EI})$. $\underline{\text { HRMS }}$ calcd for $\mathrm{C}_{12} \mathrm{H}_{10} \mathrm{O}_{2} \mathrm{Na}\left(\mathrm{M}^{+}+\right.$ Na) 209.0578 found 209.0580.

2-Methyl-6-(thiophen-2-yl)benzaldehyde (3o, Scheme 1)
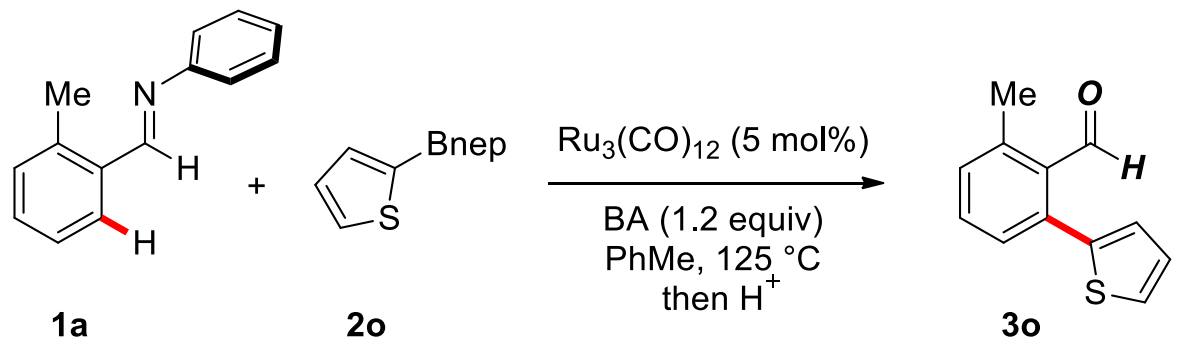

According to the general procedure A, $(E)-N$-(2-methylbenzylidene)aniline (1a, $39.1 \mathrm{mg}, 0.20$ mmol) was reacted with thiophen-2-yl-Bnep (2o, $58.8 \mathrm{mg}, 0.30 \mathrm{mmol})$, BA (35.1 mg, $0.24 \mathrm{mmol}$, 1.2 equiv) and $\mathrm{Ru}_{3}(\mathrm{CO})_{12}(6.4 \mathrm{mg}, 5 \mathrm{~mol} \%)$ in toluene $(1.0 \mathrm{M})$ at $125{ }^{\circ} \mathrm{C}$ for $1 \mathrm{~h}$. Acidic work-up 
and purification by chromatography afforded 2-methyl-6-(thiophen-2-yl)benzaldehyde in $61 \%$ yield (24.7 mg). Yellow oil. ${ }^{1} \mathbf{H}$ NMR (500 MHz, CDCl 3 ) $\delta 10.12$ (s, $\left.1 \mathrm{H}\right), 7.49-7.43$ (m, $\left.2 \mathrm{H}\right)$, 7.42-7.37 (m, $1 \mathrm{H}), 7.28(\mathrm{~d}, J=7.6 \mathrm{~Hz}, 1 \mathrm{H}), 7.16-7.10(\mathrm{~m}, 1 \mathrm{H}), 7.02(\mathrm{~d}, J=2.2 \mathrm{~Hz}, 1 \mathrm{H}), 2.64$ $(\mathrm{s}, 3 \mathrm{H}) . \underline{{ }^{13} \mathrm{C} \text { NMR }(125 \text { MHz, CDCl}} 3$ ) $\delta 194.4,140.1,140.0,139.0,133.5,132.2,131.7,129.9$, 129.3, 127.8, 127.3, 21.5. $\underline{\mathbf{M S}}=202.0$ (EI). $\underline{\mathbf{H R M S}}$ calcd for $\mathrm{C}_{12} \mathrm{H}_{10} \mathrm{OSNa}\left(\mathrm{M}^{+}+\mathrm{Na}\right) 225.0350$ found 225.0352 .

(E)-2-Methyl-6-styrylbenzaldehyde (3p, Scheme 1)<smiles>Cc1ccccc1/C=N/c1ccccc1</smiles>

$1 \mathrm{a}$

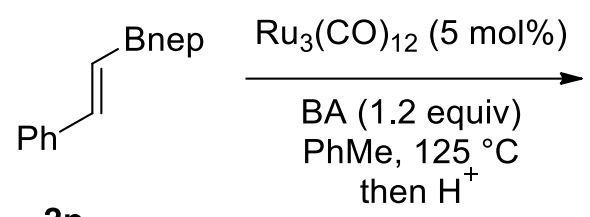

$2 p$

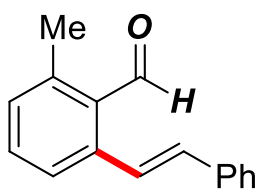

$3 p$

According to the general procedure A, $(E)-N$-(2-methylbenzylidene)aniline (1a, $39.1 \mathrm{mg}, 0.20$ mmol) was reacted with (E)-styryl-Bnep (2p, $64.8 \mathrm{mg}, 0.30 \mathrm{mmol})$, BA (35.1 $\mathrm{mg}, 0.24 \mathrm{mmol}$, 1.2 equiv) and $\mathrm{Ru}_{3}(\mathrm{CO})_{12}(6.4 \mathrm{mg}, 5 \mathrm{~mol} \%)$ in toluene $(1.0 \mathrm{M})$ at $125{ }^{\circ} \mathrm{C}$ for $1 \mathrm{~h}$. Acidic work-up and purification by chromatography afforded $(E)$-2-methyl-6-styrylbenzaldehyde in $72 \%$ yield

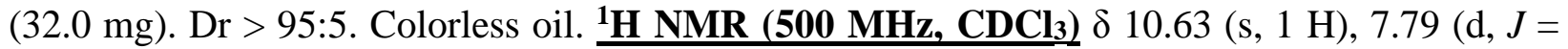
$16.1 \mathrm{~Hz}, 1 \mathrm{H}), 7.55(\mathrm{~d}, J=7.5 \mathrm{~Hz}, 2 \mathrm{H}), 7.50(\mathrm{~d}, J=7.6 \mathrm{~Hz}, 1 \mathrm{H}), 7.44(\mathrm{t}, J=7.6 \mathrm{~Hz}, 1 \mathrm{H}), 7.39$ (t, $J=7.4 \mathrm{~Hz}, 2 \mathrm{H}), 7.31(\mathrm{t}, J=7.2 \mathrm{~Hz}, 1 \mathrm{H}), 7.20(\mathrm{~d}, J=7.3 \mathrm{~Hz}, 1 \mathrm{H}), 6.94(\mathrm{~d}, J=16.1 \mathrm{~Hz}, 1 \mathrm{H})$,

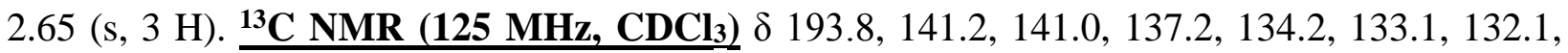
131.1, 128.9, 128.3, 127.0, 126.4, 126.1, 20.7. $\underline{\mathbf{M S}}=222.1$ (EI). $\underline{\text { HRMS }}$ calcd for $\mathrm{C}_{16} \mathrm{H}_{14} \mathrm{ONa}$ $\left(\mathrm{M}^{+}+\mathrm{Na}\right) 245.0942$ found 245.0943.

2,6-Dimethylbenzaldehyde (3q, Scheme 1)

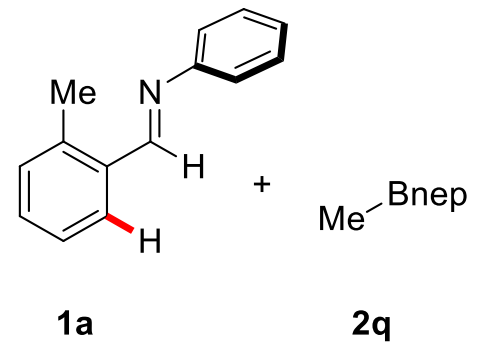

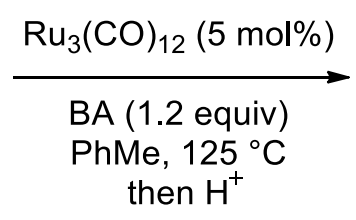

SI-15 
According to the general procedure A, (E)- $N$-(2-methylbenzylidene)aniline (1a, $39.1 \mathrm{mg}, 0.20$ mmol) was reacted with Me-Bnep (2q, $38.4 \mathrm{mg}, 0.30 \mathrm{mmol})$, BA (35.1 mg, $0.24 \mathrm{mmol}, 1.2$ equiv) and $\mathrm{Ru}_{3}(\mathrm{CO})_{12}(6.4 \mathrm{mg}, 5 \mathrm{~mol} \%)$ in toluene $(1.0 \mathrm{M})$ at $125^{\circ} \mathrm{C}$ for $8 \mathrm{~h}$. Acidic work-up and purification by chromatography afforded 2,6-dimethylbenzaldehyde in 70\% yield (18.8 mg). Caution: the product is volatile. White solid. $\mathrm{Mp}=29-30{ }^{\circ} \mathrm{C} . \underline{{ }^{1} \mathbf{H ~ N M R}\left(500 \mathbf{~ M H z}, \mathbf{C D C l}_{3}\right)} \delta$ 10.64 (s, $1 \mathrm{H}), 7.33$ (t, $J=7.5 \mathrm{~Hz}, 1 \mathrm{H}), 7.10$ (d, $J=7.5 \mathrm{~Hz}, 2 \mathrm{H}), 2.62$ (s, $6 \mathrm{H}) . \underline{{ }^{13} \mathbf{C} \text { NMR (125 }}$ $\underline{\text { MHz, } \mathbf{C D C l}}$ ) $\delta 193.8,141.3,133.2,132.7,129.9,20.7 . \underline{\mathbf{M S}}=134.1$ (EI). $\underline{\text { HRMS }}$ calcd for $\mathrm{C}_{9} \mathrm{H}_{10} \mathrm{ONa}\left(\mathrm{M}^{+}+\mathrm{Na}\right) 157.0629$ found 157.0630 . 


\section{Ru(0)-Catalyzed C-H Arylation: Variation of Imine (Scheme 2)}

\section{3-(Trifluoromethyl)-[1,1'-biphenyl]-2-carbaldehyde (3r, Scheme 2)}
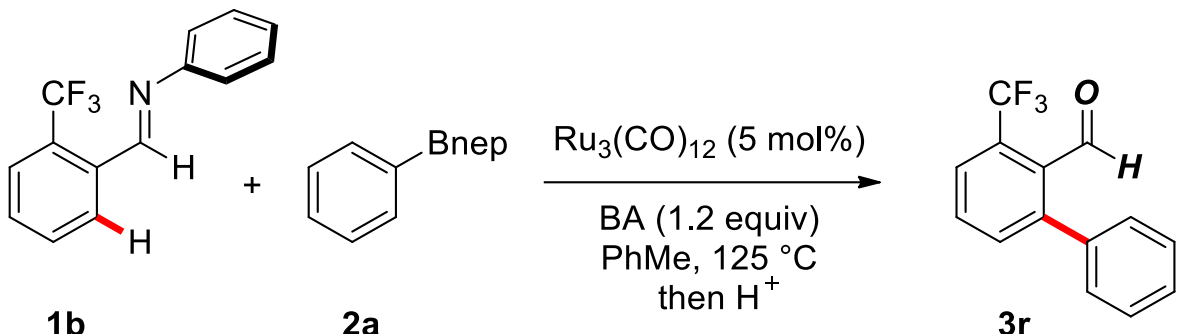

According to the general procedure A, $(E)-N$-(2-(trifluoromethyl)benzylidene)aniline (1), 49.8 mg, $0.20 \mathrm{mmol}$ ) was reacted with PhBnep (2a, $57.0 \mathrm{mg}, 0.30 \mathrm{mmol})$, BA (35.1 mg, $0.24 \mathrm{mmol}$, 1.2 equiv) and $\mathrm{Ru}_{3}(\mathrm{CO})_{12}(6.4 \mathrm{mg}, 5 \mathrm{~mol} \%)$ in toluene $(1.0 \mathrm{M})$ at $125{ }^{\circ} \mathrm{C}$ for $1 \mathrm{~h}$. Acidic work-up and purification by chromatography afforded 3-(trifluoromethyl)-[1,1'-biphenyl]-2-carbaldehyde in $85 \%$ yield (42.5 mg). Colorless oil. ${ }^{1} \mathbf{H}$ NMR (500 MHz, $\left.\mathbf{C D C l}_{3}\right) \delta 10.12(\mathrm{q}, J=1.6 \mathrm{~Hz}, 1 \mathrm{H})$, $7.83(\mathrm{~d}, J=7.7 \mathrm{~Hz}, 1 \mathrm{H}), 7.69$ (t, $J=7.8 \mathrm{~Hz}, 1 \mathrm{H}), 7.63$ (d, $J=7.6 \mathrm{~Hz}, 1 \mathrm{H}), 7.50-7.43$ (m, $3 \mathrm{H})$, 7.36-7.29 (m, 2 H). ${ }^{13} \mathbf{C}$ NMR (125 MHz, $\left.\mathbf{C D C l}_{3}\right) \delta 192.1,145.4,134.7,134.4,134.0,131.6$, $129.9,129.1$ (q, $J=32.4 \mathrm{~Hz}), 128.8,128.7,126.1(\mathrm{q}, J=6.0 \mathrm{~Hz}), 123.6(\mathrm{q}, J=274.4 \mathrm{~Hz}) . \underline{{ }^{19} \mathbf{F}}$ $\left.\underline{\text { NMR }(470 ~ M H z, ~} \mathbf{C D C l}_{3}\right) \delta-57.6(\mathrm{~s}, 3 \mathrm{~F}) . \underline{\mathbf{M S}}=250.1(\mathrm{EI}) . \underline{\text { HRMS }}$ calcd for $\mathrm{C}_{14} \mathrm{H}_{9} \mathrm{~F}_{3} \mathrm{ONa}\left(\mathrm{M}^{+}\right.$ + Na) 273.0503 found 273.0506

3-Methoxy-[1,1'-biphenyl]-2-carbaldehyde (3s, Scheme 2)
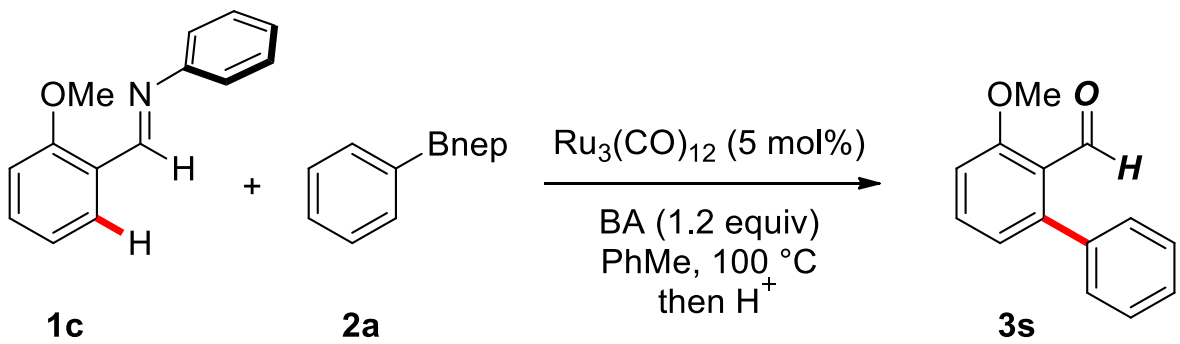

According to the general procedure A, $(E)-N$-(2-methoxybenzylidene)aniline (1c, $42.3 \mathrm{mg}, 0.20$ mmol) was reacted with PhBnep (2a, $38.0 \mathrm{mg}, 0.20 \mathrm{mmol}$ ), BA (35.1 mg, $0.24 \mathrm{mmol}, 1.2$ equiv) and $\mathrm{Ru}_{3}(\mathrm{CO})_{12}(6.4 \mathrm{mg}, 5 \mathrm{~mol} \%)$ in toluene $(1.0 \mathrm{M})$ at $100{ }^{\circ} \mathrm{C}$ for $15 \mathrm{~h}$. Acidic work-up and purification by chromatography afforded 3-methoxy-[1,1'-biphenyl]-2-carbaldehyde in 70\% yield (29.7 mg). White solid. Mp = 99-101 $\left.{ }^{\circ} \mathrm{C} . \underline{{ }^{1} \mathbf{H} \text { NMR (500 MHz, CDCl}} 3\right) \delta 10.10(\mathrm{~s}, 1 \mathrm{H})$, $7.54(\mathrm{t}, J=8.0 \mathrm{~Hz}, 1 \mathrm{H}), 7.45-7.38(\mathrm{~m}, 3 \mathrm{H}), 7.34-7.31(\mathrm{~m}, 2 \mathrm{H}), 7.02(\mathrm{~d}, J=8.4 \mathrm{~Hz}, 1 \mathrm{H}), 6.98$ 


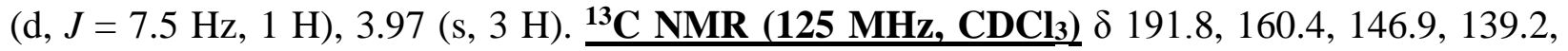
134.0, 129.8, 128.3, 128.0, 123.7, 123.3, 110.9, 56.2. $\underline{\mathbf{M S}}=212.1$ (EI). $\underline{\text { HRMS }}$ calcd for $\mathrm{C}_{14} \mathrm{H}_{12} \mathrm{O}_{2} \mathrm{Na}\left(\mathrm{M}^{+}+\mathrm{Na}\right) 235.0735$ found 235.0736.

3-Fluoro-[1,1'-biphenyl]-2-carbaldehyde (3t, Scheme 2)

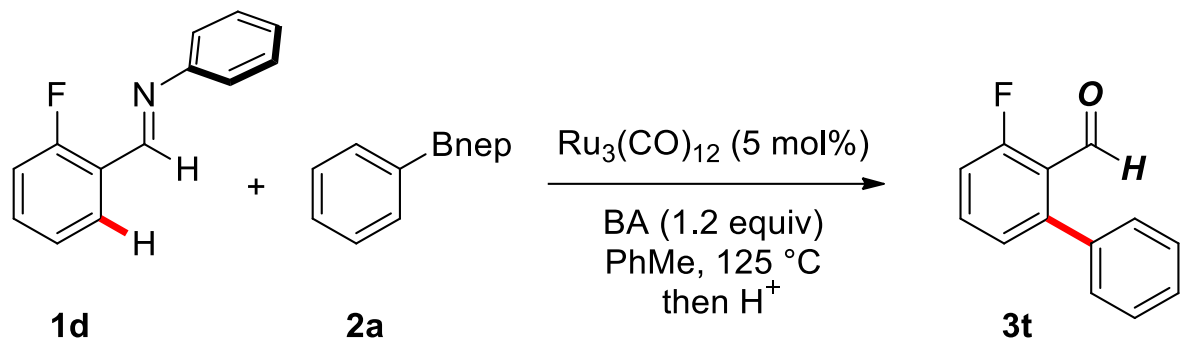

According to the general procedure A, $(E)-N$-(2-fluorobenzylidene)aniline (1d, $39.8 \mathrm{mg}, 0.20$ mmol) was reacted with PhBnep (2a, $57.0 \mathrm{mg}, 0.30 \mathrm{mmol})$, BA (35.1 mg, $0.24 \mathrm{mmol}, 1.2$ equiv) and $\mathrm{Ru}_{3}(\mathrm{CO})_{12}(6.4 \mathrm{mg}, 5 \mathrm{~mol} \%)$ in toluene $(1.0 \mathrm{M})$ at $125{ }^{\circ} \mathrm{C}$ for $15 \mathrm{~h}$. Acidic work-up and purification by chromatography afforded 3-fluoro-[1,1'-biphenyl]-2-carbaldehyde in $86 \%$ yield (34.4 mg). Colorless oil. ${ }^{1} \mathbf{H}$ NMR (500 MHz, CDCl 3 ) $\delta 9.97(s, 1 \mathrm{H}), 7.59$ (td, $J=8.0,5.4 \mathrm{~Hz}, 1$ H), 7.48-7.45 (m, $2 \mathrm{H}), 7.38-7.34(\mathrm{~m}, 2 \mathrm{H}), 7.23(\mathrm{~d}, J=7.6 \mathrm{~Hz}, 1 \mathrm{H}), 7.21-7.16(\mathrm{~m}, 1 \mathrm{H}) .{ }^{{ }^{13} \mathbf{C}}$ $\underline{\text { NMR (125 MHz, CDCl }}$ ) $\delta 189.6,162.2(\mathrm{~d}, J=262.8 \mathrm{~Hz}), 147.2,137.5$ (d, $J=2.3 \mathrm{~Hz}), 134.5(\mathrm{~d}$, $J=10.3 \mathrm{~Hz}), 130.0,128.68,128.65,126.8(\mathrm{~d}, J=3.7 \mathrm{~Hz}), 122.9(\mathrm{~d}, J=7.4 \mathrm{~Hz}), 116.0(\mathrm{~d}, J=$ $\left.21.5 \mathrm{~Hz}) . \underline{{ }^{19} \mathbf{F} \text { NMR (470 MHz, CDCl}} 3\right)$ $\mathrm{C}_{13} \mathrm{H}_{9} \mathrm{FONa}\left(\mathrm{M}^{+}+\mathrm{Na}\right) 223.0535$ found 223.0536 .

4-Methyl-[1,1'-biphenyl]-2-carbaldehyde (3u, Scheme 2)

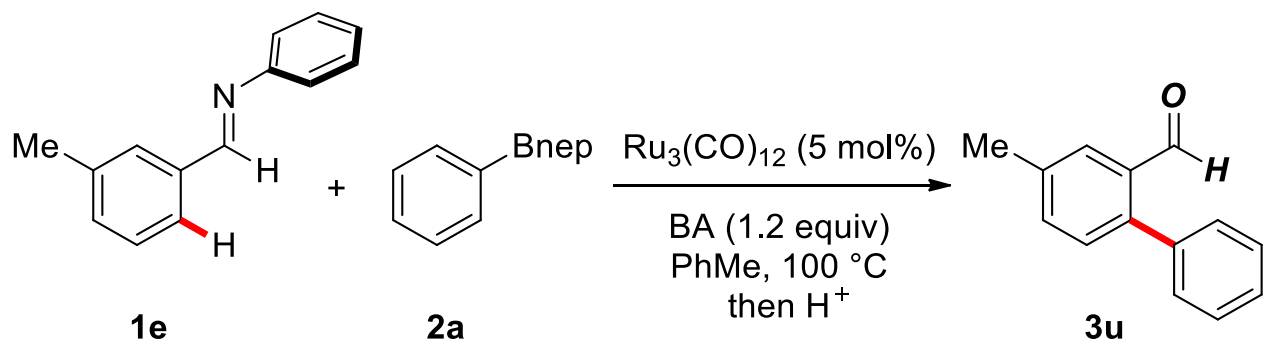

According to the general procedure A, $(E)-N$-(3-methylbenzylidene)aniline (1e, $39.0 \mathrm{mg}, 0.20$ mmol) was reacted with PhBnep (2a, $38.0 \mathrm{mg}, 0.20 \mathrm{mmol})$, BA (35.1 mg, $0.24 \mathrm{mmol}$, 1.2 equiv) and $\mathrm{Ru}_{3}(\mathrm{CO})_{12}(6.4 \mathrm{mg}, 5 \mathrm{~mol} \%)$ in toluene $(1.0 \mathrm{M})$ at $100{ }^{\circ} \mathrm{C}$ for $15 \mathrm{~h}$. Selectivity $($ monosubstituted/disubstituted $)=77: 23$. Acidic work-up and purification by chromatography SI-18 
afforded 4-methyl-[1,1'-biphenyl]-2-carbaldehyde in 60\% yield (23.5 mg). White solid. Mp = 70-

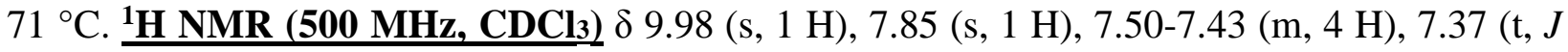
$=7.5 \mathrm{~Hz}, 3 \mathrm{H}), 2.47$ (s, $3 \mathrm{H}) .{ }^{13} \mathbf{C}$ NMR (125 MHz, $\left.\mathbf{C D C l}_{3}\right) \delta 192.9,143.6,138.0,137.9,134.7$, 133.7, 130.9, 130.3, 128.6, 128.1, 127.9, 21.2. $\underline{\mathbf{M S}}=196.1$ (EI). $\underline{\text { HRMS }}$ calcd for $\mathrm{C}_{14} \mathrm{H}_{12} \mathrm{ONa}$ $\left(\mathrm{M}^{+}+\mathrm{Na}\right) 219.0786$ found 219.0788.

4-(Trifluoromethyl)-[1,1'-biphenyl]-2-carbaldehyde (3v, Scheme 2)

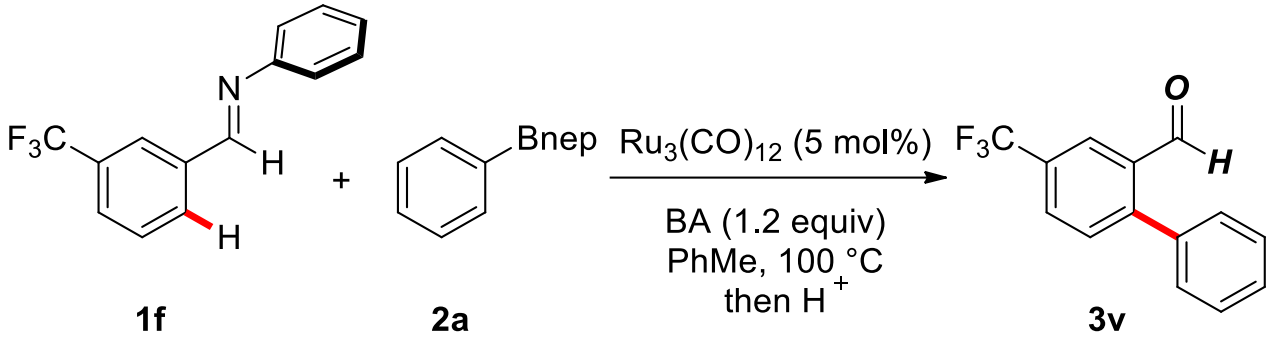

According to the general procedure A, $(E)-N$-(3-(trifluoromethyl)benzylidene)aniline (1f, 49.8 mg, $0.20 \mathrm{mmol}$ ) was reacted with PhBnep (2a, $38.0 \mathrm{mg}, 0.20 \mathrm{mmol})$, BA (35.1 mg, $0.24 \mathrm{mmol}$, 1.2 equiv) and $\mathrm{Ru}_{3}(\mathrm{CO})_{12}(6.4 \mathrm{mg}, 5 \mathrm{~mol} \%)$ in toluene $(1.0 \mathrm{M})$ at $100{ }^{\circ} \mathrm{C}$ for $1 \mathrm{~h}$. Selectivity $($ monosubstituted/disubstituted $)=60.8: 1.0$. Acidic work-up and purification by chromatography afforded 4-(trifluoromethyl)-[1,1'-biphenyl]-2-carbaldehyde in 84\% yield (42.0 mg). Yellow oil. $\underline{{ }^{1} \mathbf{H} \text { NMR (500 MHz, CDCl}}$ ) $\delta 10.01(\mathrm{~s}, 1 \mathrm{H}), 8.31(\mathrm{~s}, 1 \mathrm{H}), 7.89(\mathrm{dd}, J=8.0,1.5 \mathrm{~Hz}, 1 \mathrm{H}), 7.61$ $(\mathrm{d}, J=8.0 \mathrm{~Hz}, 1 \mathrm{H}), 7.56-7.48(\mathrm{~m}, 3 \mathrm{H}), 7.44-7.37(\mathrm{~m}, 2 \mathrm{H}) .{ }^{13} \mathbf{C}$ NMR (125 MHz, CDCl 3$)$ 191.1, 149.1, 136.6, 134.1, 131.7, 130.6 (q, $J=33.4 \mathrm{~Hz}), 130.1,130.6$ (d, $J=33.4 \mathrm{~Hz}), 129.1$, 129.0, $125.1(\mathrm{~d}, J=3.8 \mathrm{~Hz}), 123.8(\mathrm{~d}, J=272.4 \mathrm{~Hz}) .{ }^{19} \mathbf{F}$ NMR (470 MHz, CDCl 3$) \delta-62.8(\mathrm{~s}, 3$

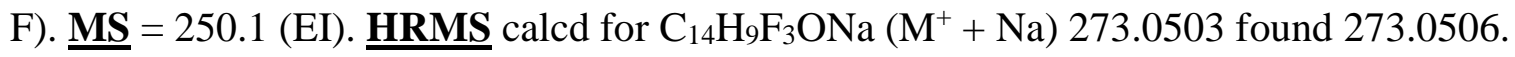

4'-Methoxy-[1,1':3',1'-terphenyl]-2' -carbaldehyde (3w, Scheme 2)<smiles>COc1cccc(/C=N/c2ccccc2)c1/C=N/c1ccccc1</smiles> 
According to the general procedure A, $(E)-N$-(3-methoxybenzylidene)aniline (1g, $42.2 \mathrm{mg}, 0.20$ mmol) was reacted with PhBnep (2a, $114.0 \mathrm{mg}, 0.60 \mathrm{mmol})$, BA (35.1 mg, 0.24mmol, 1.2 equiv) and $\mathrm{Ru}_{3}(\mathrm{CO})_{12}(6.4 \mathrm{mg}, 5 \mathrm{~mol} \%)$ in toluene $(1.0 \mathrm{M})$ at $125{ }^{\circ} \mathrm{C}$ for $8 \mathrm{~h}$. Selectivity $($ monosubstituted/disubstituted $)=1.0: 20.1$. Acidic work-up and purification by chromatography afforded 4'-methoxy-[1,1':3',1"-terphenyl]-2'-carbaldehyde in 85\% yield (49.0 mg). White solid.

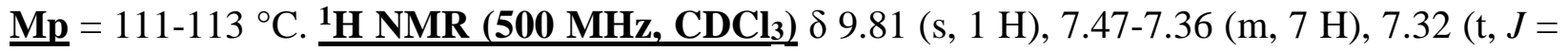
$8.1 \mathrm{~Hz}, 4 \mathrm{H}), 7.20$ (d, $J=8.5 \mathrm{~Hz}, 1 \mathrm{H}), 3.82$ (s, $3 \mathrm{H}) .{ }^{13} \mathbf{C}$ NMR (125 MHz, CDCl 3$)$ 156.6, 139.9, 136.1, 134.98, 134.96, 132.8, 131.5, 130.7, 129.8, 128.3, 128.1, 127.7, 127.4, 114.5, 56.3. $\underline{\mathbf{M S}}=288.1(\mathrm{EI}) . \underline{\mathbf{H R M S}}$ calcd for $\mathrm{C}_{20} \mathrm{H}_{16} \mathrm{O}_{2} \mathrm{Na}\left(\mathrm{M}^{+}+\mathrm{Na}\right) 311.1048$ found 311.1049 .

\section{4-Fluoro-[1,1'-biphenyl]-2-carbaldehyde (3t, Scheme 2)}

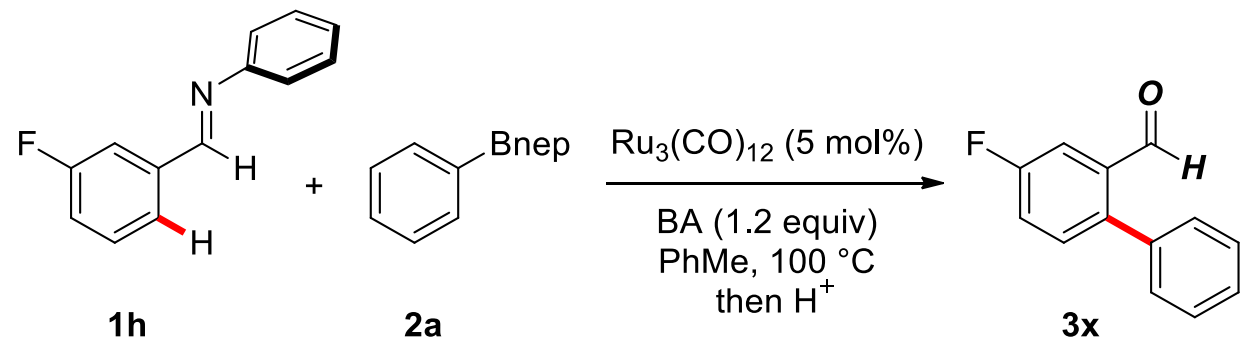

According to the general procedure A, $(E)-N$-(3-fluorobenzylidene)aniline (1h, $39.8 \mathrm{mg}, 0.20$ mmol) was reacted with PhBnep (2a, $38.0 \mathrm{mg}, 0.20 \mathrm{mmol}$ ), BA (35.1 mg, $0.24 \mathrm{mmol}, 1.2$ equiv) and $\mathrm{Ru}_{3}(\mathrm{CO})_{12}(6.4 \mathrm{mg}, 5 \mathrm{~mol} \%)$ in toluene $(1.0 \mathrm{M})$ at $100{ }^{\circ} \mathrm{C}$ for $15 \mathrm{~h}$. Selectivity $($ monosubstituted/disubstituted $)=71: 29$. Acidic work-up and purification by chromatography afforded 4-fluoro-[1,1'-biphenyl]-2-carbaldehyde in 51\% yield (20.4 mg). Colorless oil. $\underline{\mathbf{H}}$ NMR (500 MHz, CDCl $)$ ) $9.83(\mathrm{~s}, 1 \mathrm{H}), 7.85(\mathrm{~d}, J=7.7 \mathrm{~Hz}, 1 \mathrm{H}), 7.54-7.46(\mathrm{~m}, 4 \mathrm{H})$, 7.44-

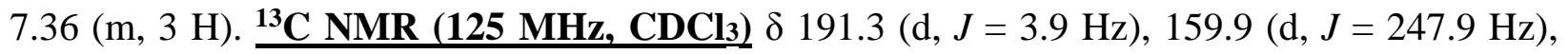
$136.0(\mathrm{~d}, J=2.3 \mathrm{~Hz}), 133.17(\mathrm{~d}, J=16.8 \mathrm{~Hz}), 131.1$ (d, $J=1.1 \mathrm{~Hz}), 130.6,129.3$ (d, $J=8.1 \mathrm{~Hz})$, 128.9, 128.6, $123.4(\mathrm{~d}, J=3.5 \mathrm{~Hz}), 121.1(\mathrm{~d}, J=23.1 \mathrm{~Hz}) . \underline{\left.{ }^{19} \mathbf{F} \text { NMR (470 MHz, CDCl }\right)} \delta$ $116.0(\mathrm{~m}, 1 \mathrm{~F}) . \underline{\mathbf{M S}}=200.1(\mathrm{EI})$. $\underline{\mathbf{H R M S}}$ calcd for $\mathrm{C}_{13} \mathrm{H}_{9} \mathrm{FONa}\left(\mathrm{M}^{+}+\mathrm{Na}\right) 223.0535$ found 223.0536 .

\section{[1,1':3',1'--Terphenyl]-2'-carbaldehyde (3y, Scheme 2)}



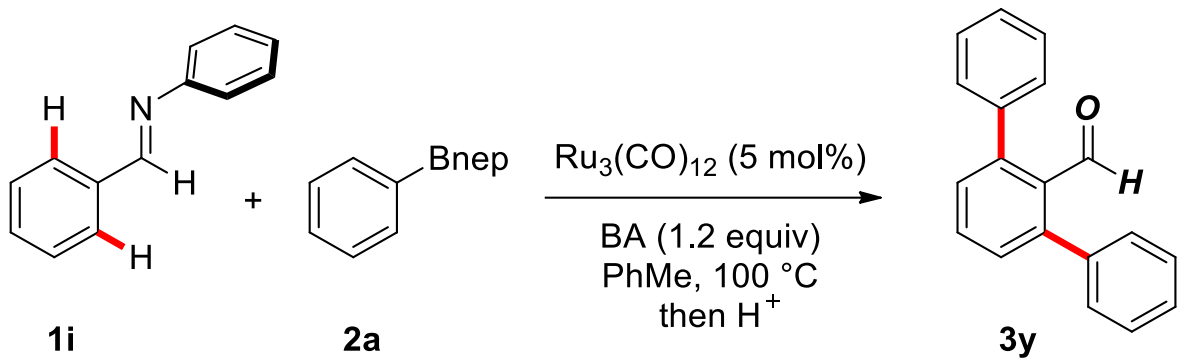

According to the general procedure A, $(E)-N$-benzylideneaniline (1i, $36.2 \mathrm{mg}, 0.20 \mathrm{mmol})$ was reacted with PhBnep (2a, $114.0 \mathrm{mg}, 0.60 \mathrm{mmol})$, BA (35.1 mg, $0.24 \mathrm{mmol}, 1.2 \mathrm{equiv})$ and $\mathrm{Ru}_{3}(\mathrm{CO})_{12}(6.4 \mathrm{mg}, 5 \mathrm{~mol} \%)$ in toluene $(1.0 \mathrm{M})$ at $125{ }^{\circ} \mathrm{C}$ for $8 \mathrm{~h}$. Selectivity $($ monosubstituted $/$ disubstituted $)=1.0 / 60.1$. Acidic work-up and purification by chromatography afforded [1,1':3',1"-terphenyl]-2'-carbaldehyde in 90\% yield (46.5 mg). White solid. $\underline{\mathbf{M p}}=122$ $123{ }^{\circ} \mathrm{C} . \underline{\mathbf{1}_{\mathbf{H}} \mathbf{N M R}\left(\mathbf{5 0 0} \mathbf{~ M H z}, \mathbf{C D C l}_{3}\right)} \delta 9.99(\mathrm{~s}, 1 \mathrm{H}), 7.61(\mathrm{t}, J=7.6 \mathrm{~Hz}, 1 \mathrm{H}), 7.49-7.40$ (m, 8 H), 7.40-7.34 (m, 4 H). ${ }^{13}$ C NMR (125 MHz, CDCl3) $\delta 193.7,144.5,139.8,133.3,131.7,130.6$, 129.8, 128.3, 127.8. $\underline{\mathbf{M S}}=258.1(\mathrm{EI})$. $\underline{\mathbf{H R M S}}$ calcd for $\mathrm{C}_{19} \mathrm{H}_{14} \mathrm{ONa}\left(\mathrm{M}^{+}+\mathrm{Na}\right) 281.0942$ found 281.0944 .

1-([1,1'-Biphenyl]-2-yl)ethanone (3z, Scheme 2)

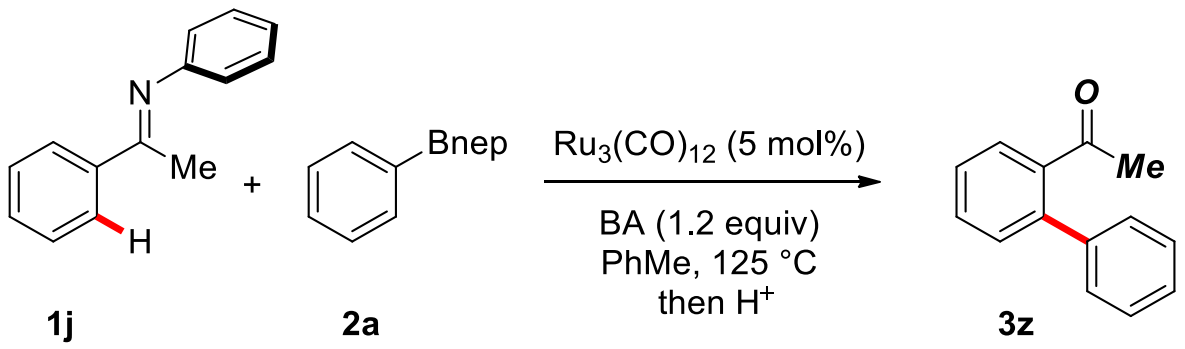

According to the general procedure A, (E)-N-(1-phenylethylidene)aniline (1j, $39.1 \mathrm{mg}, 0.20$ mmol) was reacted with PhBnep (2a, $38.0 \mathrm{mg}, 0.20 \mathrm{mmol})$, BA (35.1 mg, $0.24 \mathrm{mmol}, 1.2$ equiv) and $\mathrm{Ru}_{3}(\mathrm{CO})_{12}(6.4 \mathrm{mg}, 5 \mathrm{~mol} \%)$ in toluene $(1.0 \mathrm{M})$ at $125{ }^{\circ} \mathrm{C}$ for $1 \mathrm{~h}$. Selectivity (monosubstituted/disubstituted) $>92: 8$. Acidic work-up and purification by chromatography afforded 1-([1,1'-biphenyl]-2-yl)ethanone in 91\% yield $(35.7 \mathrm{mg})$. White solid. $\underline{\mathbf{M p}}=116$ -

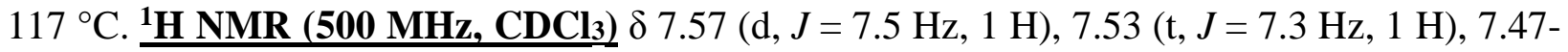
$7.39(\mathrm{~m}, 5 \mathrm{H}), 7.36(\mathrm{~d}, J=6.9 \mathrm{~Hz}, 2 \mathrm{H}), 2.02$ (s, $3 \mathrm{H}) .{ }^{13} \mathbf{C}$ NMR (125 MHz, CDCl $\left.\mathbf{3}\right) \delta 205.1$, $141.1,140.9,140.7,130.9,130.4,129.0,128.9,128.1,128.0,127.6,30.6$. MS $=196.1$ (EI). HRMS calcd for $\mathrm{C}_{14} \mathrm{H}_{12} \mathrm{ONa}\left(\mathrm{M}^{+}+\mathrm{Na}\right) 219.0786$ found 219.0788 . 
3-Phenylthiophene-2-carbaldehyde (3aa, Scheme 2)

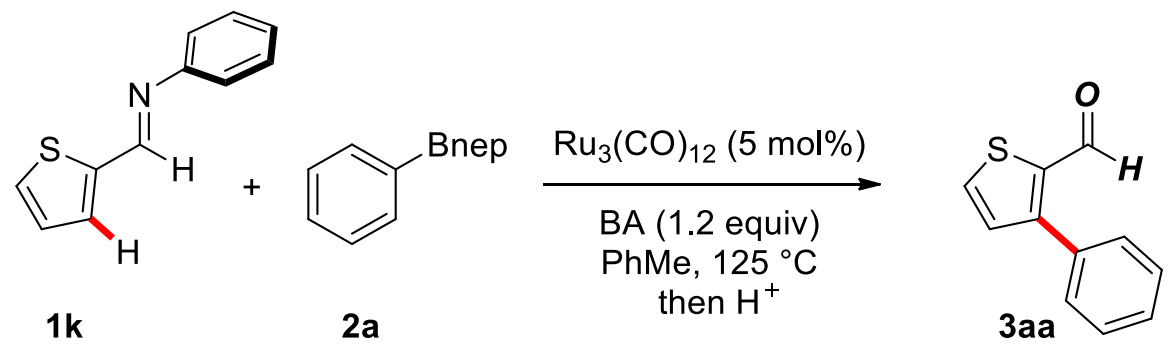

According to the general procedure A, $(E)-N$-(thiophen-2-ylmethylene)aniline $(\mathbf{1 k}, 37.5 \mathrm{mg}, 0.20$ mmol) was reacted with PhBnep (2a, $57.0 \mathrm{mg}, 0.30 \mathrm{mmol})$, BA (35.1 mg, 0.24mmol, 1.2 equiv) and $\mathrm{Ru}_{3}(\mathrm{CO})_{12}(6.4 \mathrm{mg}, 5 \mathrm{~mol} \%)$ in toluene $(1.0 \mathrm{M})$ at $125{ }^{\circ} \mathrm{C}$ for $1 \mathrm{~h}$. Acidic work-up and purification by chromatography afforded 3-phenylthiophene-2-carbaldehyde in 96\% yield (36.1

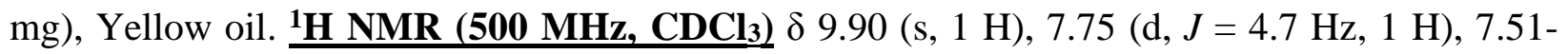

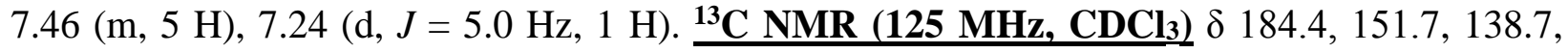
134.3, 134.1, 130.8, 129.8, 129.0, 129.0. $\underline{\mathbf{M S}}=188.0$ (EI). $\underline{\mathbf{H R M S}}$ calcd for $\mathrm{C}_{11} \mathrm{H}_{8} \mathrm{OSNa}\left(\mathrm{M}^{+}+\right.$ Na) 211.0194 found 211.0194 .

\section{2-Phenylfuran-3-carbaldehyde (3ab, Scheme 2)}

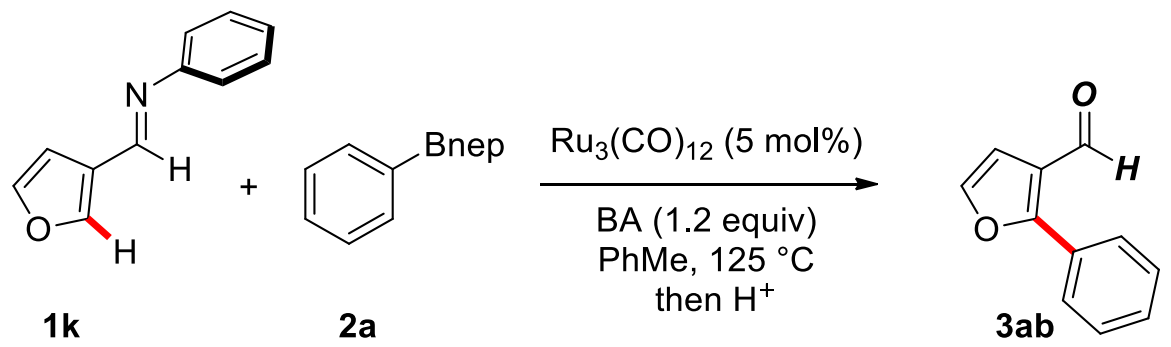

According to the general procedure C, furan-3-carbaldehyde (19.2 $\mathrm{mg}, 0.20 \mathrm{mmol})$ was reacted with aniline (18.6 mg, $0.2 \mathrm{mmol}, 1.0$ equiv), PhBnep (2a, $57.0 \mathrm{mg}, 0.30 \mathrm{mmol})$, BA (35.1 mg, $0.24 \mathrm{mmol}, 1.2$ equiv), $\mathrm{Ru}_{3}(\mathrm{CO})_{12}(6.4 \mathrm{mg}, 5 \mathrm{~mol} \%)$ and anhydrous $\mathrm{MgSO}_{4}(36 \mathrm{mg}, 0.3 \mathrm{mmol}$, 1.5 equiv) in toluene $(1.0 \mathrm{M})$ at $125{ }^{\circ} \mathrm{C}$ for $1 \mathrm{~h}$. Selectivity >95:5. Acidic work-up and purification by chromatography afforded 2-phenylfuran-3-carbaldehyde in 76\% yield (26.2 $\mathrm{mg}$ ). Yellow oil. ${ }^{1} \mathbf{H}$ NMR (500 MHz, $\left.\mathbf{C D C l}_{3}\right) \delta 10.14$ (s, 1 H), 7.75 (dd, $J=7.6,1.8$ Hz, 2 H), 7.57$7.45(\mathrm{~m}, 4 \mathrm{H}), 6.92$ (d, $J=1.8 \mathrm{~Hz}, 1 \mathrm{H}) .{ }^{\mathbf{1 3}} \mathbf{C} \mathbf{N M R}\left(\mathbf{1 2 5} \mathbf{M H z}, \mathbf{C D C l}_{3}\right) \delta 185.9,161.8,143.0$, 130.4, 129.2, 129.1, 128.3, 123.2, 109.7. $\underline{\mathbf{M S}}=172.1(\mathrm{EI})$. $\underline{\mathbf{H R M S}}$ calcd for $\mathrm{C}_{11} \mathrm{H}_{8} \mathrm{O}_{2} \mathrm{Na}\left(\mathrm{M}^{+}+\right.$ Na) 195.0422 found 195.0423 . 
1-Phenyl-2-naphthaldehyde (3ac, Scheme 2)

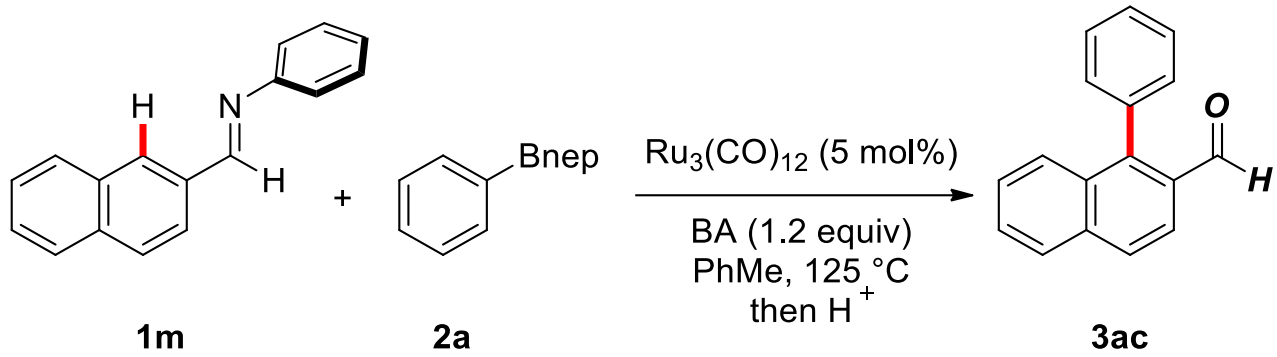

According to the general procedure A, $(E)-N$-(naphthalen-2-ylmethylene)aniline (1m, $46.2 \mathrm{mg}$, $0.20 \mathrm{mmol}$ ) was reacted with PhBnep (2a, $57.0 \mathrm{mg}, 0.30 \mathrm{mmol}$ ), BA (35.1 mg, $0.24 \mathrm{mmol}, 1.2$ equiv) and $\mathrm{Ru}_{3}(\mathrm{CO})_{12}(6.4 \mathrm{mg}, 5 \mathrm{~mol} \%)$ in toluene $(1.0 \mathrm{M})$ at $125{ }^{\circ} \mathrm{C}$ for $1 \mathrm{~h}$. Selectivity >95:5. Acidic work-up and purification by chromatography afforded 1-phenyl-2-naphthaldehyde in $96 \%$ yield (44.6 mg). White solid. $\underline{\mathbf{M p}}=120-123{ }^{\circ} \mathrm{C} . \underline{\mathbf{H}} \mathbf{\text { NMR }}(\mathbf{5 0 0} \mathbf{M H z}, \mathbf{C D C l} 3)$ $9.27(\mathrm{~d}, J=8.7 \mathrm{~Hz}, 1 \mathrm{H}), 8.10(\mathrm{~d}, J=8.4 \mathrm{~Hz}, 1 \mathrm{H}), 7.93(\mathrm{~d}, J=8.1 \mathrm{~Hz}, 1 \mathrm{H}), 7.75-7.68(\mathrm{~m}, 1 \mathrm{H})$, 7.63-7.58 (m, $1 \mathrm{H}), 7.55(\mathrm{~d}, J=8.4 \mathrm{~Hz}, 1 \mathrm{H}), 7.54-7.48(\mathrm{~m}, 3 \mathrm{H}), 7.48-7.44(\mathrm{~m}, 2 \mathrm{H}) . \underline{\mathbf{1 3}} \mathbf{\text { NMR }}$ $\underline{\left(125 \mathbf{M H z}_{2} \mathbf{C D C l}_{3}\right)} \delta 194.9,148.1,138.9,134.1,133.2,130.8,130.5,129.4,129.0,128.5,128.5$, 128.5, 127.0, 126.0. $\underline{\mathbf{M S}}=232.1$ (EI). $\underline{\mathbf{H R M S}}$ calcd for $\mathrm{C}_{17} \mathrm{H}_{12} \mathrm{ONa}\left(\mathrm{M}^{+}+\mathrm{Na}\right) 255.0786$ found 255.0788 .

[1,1':3',1''-Terphenyl]-2'-carbaldehyde (3y, Scheme 2)

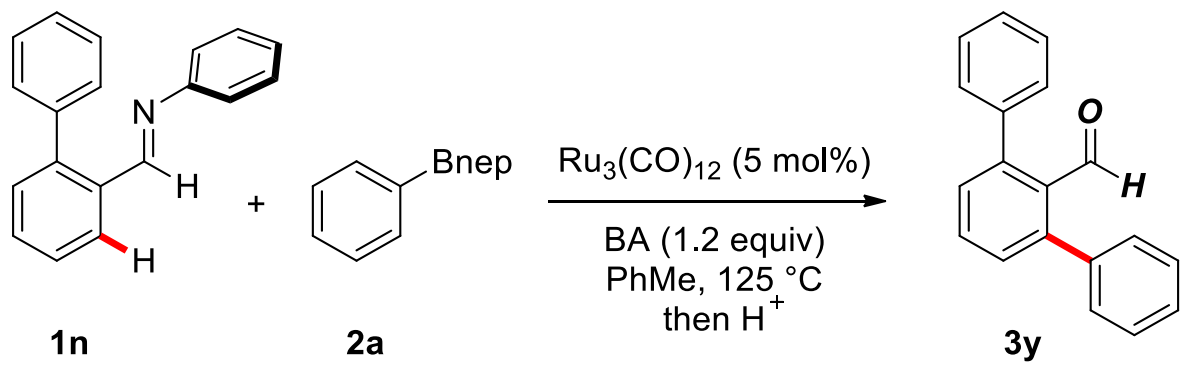

According to the general procedure $\mathrm{A},(E)-N-([1,1$ '-biphenyl]-2-ylmethylene)aniline (1n, 51.5 mg, $0.20 \mathrm{mmol}$ ) was reacted with PhBnep (2a, $57.0 \mathrm{mg}, 0.30 \mathrm{mmol})$, BA (35.1 mg, $0.24 \mathrm{mmol}$, 1.2 equiv) and $\mathrm{Ru}_{3}(\mathrm{CO})_{12}(6.4 \mathrm{mg}, 5 \mathrm{~mol} \%)$ in toluene $(1.0 \mathrm{M})$ at $125{ }^{\circ} \mathrm{C}$ for $1 \mathrm{~h}$. Acidic work-up and purification by chromatography afforded [1,1':3',1"-terphenyl]-2'-carbaldehyde in $91 \%$ yield

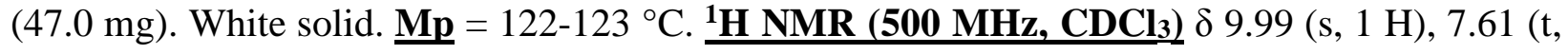
$J=7.6 \mathrm{~Hz}, 1 \mathrm{H}), 7.49-7.40(\mathrm{~m}, 8 \mathrm{H}), 7.40-7.34(\mathrm{~m}, 4 \mathrm{H}) . \underline{{ }^{13} \mathbf{C ~ N M R}(\mathbf{1 2 5} \mathbf{M H z}, \mathbf{C D C l} 3)} \delta 193.7$, 
144.5, 139.8, 133.3, 131.7, 130.6, 129.8, 128.3, 127.8. $\underline{\mathbf{M S}}=258.1$ (EI). $\underline{\text { HRMS }}$ calcd for $\mathrm{C}_{19} \mathrm{H}_{14} \mathrm{ONa}\left(\mathrm{M}^{+}+\mathrm{Na}\right) 281.0942$ found 281.0944 .

2'-Formyl-[1,1':3',1"'-terphenyl]-4'-yl 4-methylbenzenesulfonate (3ad, Scheme 2)

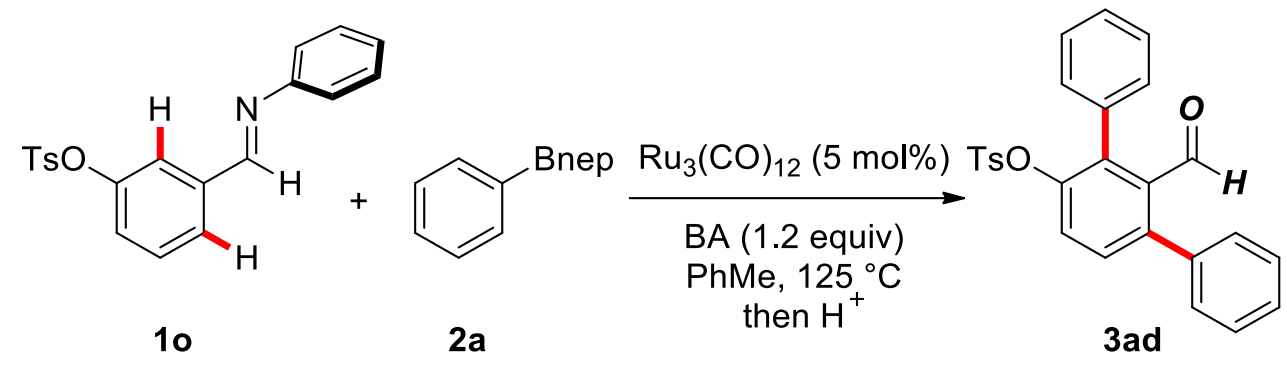

According to the general procedure C, 3-formylphenyl 4-methylbenzenesulfonate (55.3 mg, 0.20 mmol) was reacted with aniline (18.6 mg, $0.2 \mathrm{mmol}, 1.0$ equiv), PhBnep (2a, $114.0 \mathrm{mg}, 0.60$ mmol), BA (35.1 mg, $0.24 \mathrm{mmol}, 1.2$ equiv), $\mathrm{Ru}_{3}(\mathrm{CO})_{12}(6.4 \mathrm{mg}, 5 \mathrm{~mol} \%)$, and anhydrous $\mathrm{MgSO}_{4}\left(36 \mathrm{mg}, 0.3 \mathrm{mmol}, 1.5\right.$ equiv) in toluene $(1.0 \mathrm{M})$ at $125{ }^{\circ} \mathrm{C}$ for $8 \mathrm{~h}$. Selectivity $($ monosubstituted/disubstituted $)=1.0 / 12.6$. Acidic work-up and purification by chromatography afforded 2'-formyl-[1,1':3',1"-terphenyl]-4'-yl 4-methylbenzenesulfonate in 80\% yield (46.5 mg). White solid. $\left.\underline{\mathbf{M p}}=109-111^{\circ} \mathrm{C} . \underline{{ }^{1} \mathbf{H} \text { NMR (500 MHz, CDCl}} 3\right) \delta 9.72(\mathrm{~s}, 1 \mathrm{H}), 7.75(\mathrm{~d}, J=8.5 \mathrm{~Hz}$, $1 \mathrm{H})$, 7.47-7.43 (m, $4 \mathrm{H}), 7.36-7.32(\mathrm{~m}, 3 \mathrm{H}), 7.31-7.28(\mathrm{~m}, 4 \mathrm{H}), 7.14$ (d, J = 8.1 Hz, $2 \mathrm{H}), 7.04$ $(\mathrm{d}, J=7.2 \mathrm{~Hz}, 2 \mathrm{H}), 2.44(\mathrm{~s}, 3 \mathrm{H}) .{ }^{13} \mathbf{C} \mathbf{N M R}\left(125 \mathbf{M H z}, \mathbf{C D C l}_{3}\right) \delta 192.7,146.5,145.2,142.8$, $138.7,137.0,135.5,133.0,132.7,131.7,130.6,129.8,129.7,128.5,128.21,128.17,128.01$, 127.95, 126.5, 21.8. $\underline{\mathbf{M S}}=428.1(\mathrm{EI}) . \underline{\mathbf{H R M S}}$ calcd for $\mathrm{C}_{26} \mathrm{H}_{20} \mathrm{O}_{4} \mathrm{SNa}\left(\mathrm{M}^{+}+\mathrm{Na}\right) 451.0980$ found 451.0981 .

3,3-Diphenylacrylaldehyde (3ae, Scheme 2)
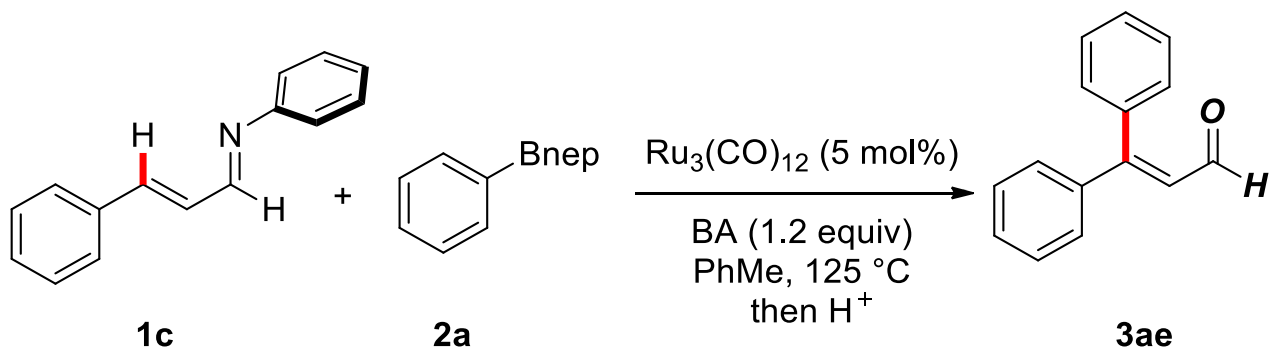

According to the general procedure A, $(E)-N-((E)$-3-phenylallylidene) aniline $(\mathbf{1 p}, 41.6 \mathrm{mg}, 0.20$ mmol) was reacted with PhBnep (2a, $38.0 \mathrm{mg}, 0.20 \mathrm{mmol})$, BA (35.1 mg, $0.24 \mathrm{mmol}, 1.2$ equiv) 
and $\mathrm{Ru}_{3}(\mathrm{CO})_{12}(6.4 \mathrm{mg}, 5 \mathrm{~mol} \%)$ in toluene $(1.0 \mathrm{M})$ at $125{ }^{\circ} \mathrm{C}$ for $1 \mathrm{~h}$. The title product was isolated after reduction to alcohol. Acidic work-up, followed by reduction with $\mathrm{NaBH}_{4} / \mathrm{EtOH}$ (EtOH, $5 \mathrm{~mL}, 1 \mathrm{~h}, 3$ equiv) and purification by chromatography afforded 3,3-diphenylprop-2-en1-ol (3ae') in 64\% yield (26.9 mg). White solid. $\underline{\mathbf{M p}}=88-89^{\circ} \mathrm{C} . \underline{\left.{ }^{1} \mathbf{H ~ N M R ~ ( 5 0 0 ~} \mathbf{~ M H z}, \mathbf{C D C l}_{3}\right)} \delta$ 7.42-7.33 (m, 3 H), 7.32-7.26 (m, 5 H), 7.21-7.15 (m, 2 H), 6.26 (t, $J=6.8 \mathrm{~Hz}, 1 \mathrm{H}), 4.24$ (d, $J=$

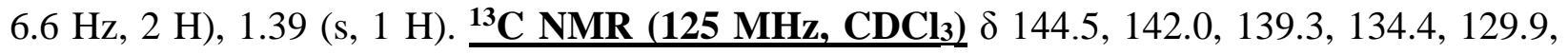
$128.42,128.38,127.80,127.77,127.6,60.9 . \underline{\mathbf{M S}}=210.1(\mathrm{EI}) . \underline{\mathbf{H R M S}}$ calcd for $\mathrm{C}_{15} \mathrm{H}_{14} \mathrm{ONa}\left(\mathrm{M}^{+}\right.$ + Na) 233.0942 found 233.0945 . 


\section{Ru(0)-Catalyzed Formal Direct Arylation of Aldehydes via in situ Imine Synthesis}
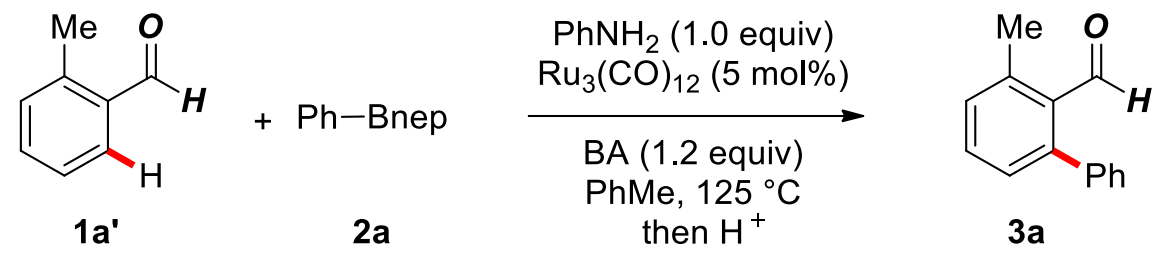

According to the general procedure $\mathrm{C}$, an oven-dried vial was charged with 2methylbenzaldehyde ( $24.0 \mathrm{mg}, 0.2 \mathrm{mmol}, 1.0$ equiv), aniline (18.6 mg, $0.2 \mathrm{mmol}, 1.0$ equiv), boronic ester (57.0 mg, 1.5 equiv), $\mathrm{H}$-acceptor $\left(35.1 \mathrm{mg}, 1.2\right.$ equiv), $\mathrm{Ru}_{3}(\mathrm{CO})_{12}(6.4 \mathrm{mg}, 5$ mol\%) and anhydrous $\mathrm{MgSO}_{4}$ (36 $\mathrm{mg}, 0.3 \mathrm{mmol}, 1.5$ equiv), placed under a positive pressure of argon, and subjected to three evacuation/backfilling cycles under high vacuum. Toluene (1.0 M) was added at room temperature, the reaction mixture was placed in a preheated oil bath at 125 ${ }^{\circ} \mathrm{C}$, and stirred for $15 \mathrm{~h}$ at $125{ }^{\circ} \mathrm{C}$. After the indicated time, the reaction mixture was cooled down to room temperature, and subjected to standard imine deprotection procedure (diethyl ether, $\mathrm{HCl}_{\mathrm{aq}}, 1 \mathrm{~N}, 3 \mathrm{~h}$, room temperature). Purification by chromatography on silica gel afforded the title product. Yield $77 \%$. Spectroscopic data matched those described previously. 


\section{Mechanistic Selectivity Studies - Imine Electronics}

General Procedure. An oven-dried vial equipped with a stir bar was charged with two imine substrates (each $0.4 \mathrm{mmol}, 2.0$ equiv), PhBnep (2a, $38.0 \mathrm{mg}, 0.20 \mathrm{mmol}, 1.0$ equiv), BA (35.1 mg, $0.24 \mathrm{mmol}, 1.2$ equiv), $\mathrm{Ru}_{3}(\mathrm{CO})_{12}(6.4 \mathrm{mg}, 5 \mathrm{~mol} \%)$, placed under a positive pressure of argon, and subjected to three evacuation/backfilling cycles under high vacuum. Toluene (1.0 M) was added at room temperature, the reaction mixture was placed in a preheated oil bath at 125 ${ }^{\circ} \mathrm{C}$, and stirred for $15 \mathrm{~h}$ at $125{ }^{\circ} \mathrm{C}$. After the indicated time, the reaction mixture was cooled down to room temperature. The sample was analyzed by ${ }^{1} \mathrm{H} \mathrm{NMR}\left(\mathrm{CDCl}_{3}, 500 \mathrm{MHz}\right)$ and GCMS to obtain conversion and yield using internal standard and comparison with authentic samples.

Table SI-1. Selectivity Study in the Ru(0)-Catalyzed C-H Arylation of Imines. ${ }^{a}$<smiles>[R7]c1cccc(/C=N/c2ccccc2)c1</smiles><smiles>[R2]c1cccc(/C=N/c2ccccc2)c1</smiles>

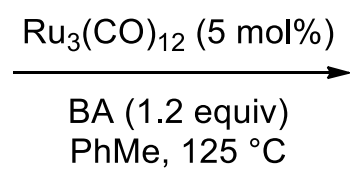<smiles>[R]c1ccc(-c2ccccc2)c(/N=C/c2ccccc2)c1</smiles><smiles>[R2]c1ccc(-c2ccccc2)c(/C=N/c2ccccc2)c1</smiles>
$1-$ II 3-II

\begin{tabular}{ccccc}
\hline Entry & $\mathbf{1 - I}$ & $\mathbf{1 - I I}$ & Bnep & 3-I:3-II \\
& $\left(\mathrm{R}_{1}\right)$ & $\left(\mathrm{R}_{2}\right)$ & $($ equiv $)$ & $\left(\mathrm{R}_{1}: \mathrm{R}_{2}\right)^{b}$ \\
\hline 1 & $\mathrm{CF}_{3-}$ & $\mathrm{Me}-$ & 0.5 & $3.04: 1$ \\
2 & $\mathrm{CF}_{3-}$ & $\mathrm{MeO}-$ & 0.5 & $4.63: 1$ \\
\hline
\end{tabular}

${ }^{a}$ All reactions carried out using standard Schlenk techniques under argon. Conditions: imine (0.4 mmol, 2.0 equiv), PhBnep (2a, $0.20 \mathrm{mmol}, 1.0$ equiv), BA ( $0.24 \mathrm{mmol}, 1.2$ equiv), $\mathrm{Ru}_{3}(\mathrm{CO})_{12}(0.10 \mathrm{mmol}), 125{ }^{\circ} \mathrm{C}, 15 \mathrm{~h} .{ }^{b}$ Determined by ${ }^{1} \mathrm{H} \mathrm{NMR}$ and/or GCMS. 


\section{Mechanistic Selectivity Studies - Organoborane}

General Procedure. An oven-dried vial equipped with a stir bar was charged with an imine substrate ( $0.2 \mathrm{mmol}, 1.0$ equiv), two aryl boronic ester substrates (each $0.40 \mathrm{mmol}, 2.0$ equiv), BA (35.1 mg, $0.24 \mathrm{mmol}, 1.2$ equiv), $\mathrm{Ru}_{3}(\mathrm{CO})_{12}(6.4 \mathrm{mg}, 5 \mathrm{~mol} \%)$, placed under a positive pressure of argon, and subjected to three evacuation/backfilling cycles under high vacuum. Toluene $(1.0 \mathrm{M})$ was added at room temperature, the reaction mixture was placed in a preheated oil bath at $125{ }^{\circ} \mathrm{C}$, and stirred for $15 \mathrm{~h}$ at $125{ }^{\circ} \mathrm{C}$. After the indicated time, the reaction mixture was cooled down to room temperature. The sample was analyzed by ${ }^{1} \mathrm{H} \mathrm{NMR}\left(\mathrm{CDCl}_{3}, 500\right.$ $\mathrm{MHz}$ ) and GC-MS to obtain conversion and yield using internal standard and comparison with authentic samples..

Table SI-2. Selectivity Study in the Ru(0)-Catalyzed C-H Arylation of Imines. ${ }^{a}$<smiles>Cc1ccccc1/C=N/c1ccccc1</smiles><smiles>[R7]c1ccc(Br)cc1</smiles>

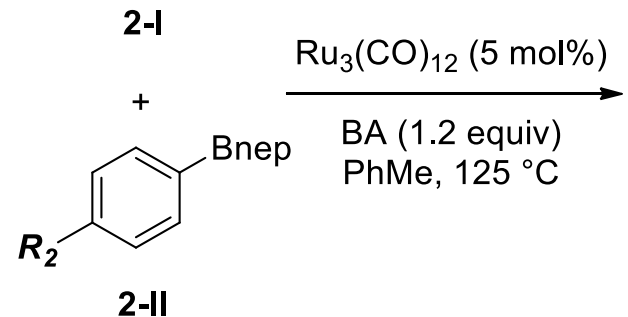<smiles>[R2]c1ccc(-c2cccc(C)c2/C=N/c2ccccc2)cc1</smiles>

\begin{tabular}{ccccc}
\hline Entry & $\mathbf{2 - I}$ & $\mathbf{2 - I I}$ & imine & 3-I:3-II \\
& $\left(\mathrm{R}_{1}\right)$ & $\left(\mathrm{R}_{2}\right)$ & $($ equiv $)$ & $\left(\mathrm{R}_{1}: \mathrm{R}_{2}\right)^{b}$ \\
\hline 1 & $\mathrm{MeO}$ & $\mathrm{CF}_{3-}$ & 0.5 & $1.0: 1.08$
\end{tabular}

${ }^{a}$ All reactions carried out using standard Schlenk techniques under argon. Conditions: imine (0.2 mmol, 1.0 equiv), ArBnep (0.40 mmol, 2.0 equiv), BA $\left(0.24 \mathrm{mmol}, 1.2\right.$ equiv), $\mathrm{Ru}_{3}(\mathrm{CO}){ }_{12}(0.10 \mathrm{mmol}), 125^{\circ} \mathrm{C}, 15 \mathrm{~h} .{ }^{b}$ Determined by ${ }^{1} \mathrm{H}$ NMR and/or GC-MS. 


\section{Product Transformations}

1-Phenyl-2-naphthoic acid (4a, Oxidation, Product A)<smiles>O=Cc1ccc2ccccc2c1-c1ccccc1</smiles>

3ac

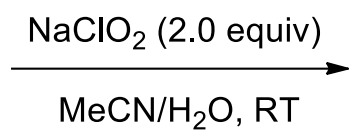

An oven-dried vial equipped with a stir bar was charged with 1-phenyl-2-naphthaldehyde (3ac, $23.2 \mathrm{mg}, 0.1 \mathrm{mmol}), \mathrm{NaClO}_{2}(18.1 \mathrm{mg}, 0.2 \mathrm{mmol}), \mathrm{MeCN}(1.0 \mathrm{~mL})$ and $\mathrm{H}_{2} \mathrm{O}(1.0 \mathrm{~mL})$, and the resulting reaction mixture was stirred at room temperature for $18 \mathrm{~h}$. After the indicated time, the reaction mixture was diluted with $\mathrm{Na}_{2} \mathrm{SO}_{3}$ (aq., $10 \mathrm{~mL}$ ), and extracted with dichloromethane $(3 \mathrm{x}$ $10 \mathrm{~mL}$ ). The organic layers were combined, dried over $\mathrm{Na}_{2} \mathrm{SO}_{4}$, filtered and concentrated. Purification by chromatography on silica gel afforded the title product. Yield 76\% (18.9 $\mathrm{mg}$ ). White solid. $\underline{\mathbf{M p}}=123-125^{\circ} \mathrm{C} . \underline{{ }^{1} \mathbf{H} \text { NMR }\left(\mathbf{5 0 0} \mathbf{~ M H z}, \mathbf{C D C l}_{3}\right)} \delta 8.10(\mathrm{~d}, J=8.3 \mathrm{~Hz}, 1 \mathrm{H}), 8.00(\mathrm{~d}$, $J=8.5 \mathrm{~Hz}, 1 \mathrm{H}), 7.93(\mathrm{~d}, J=8.0 \mathrm{~Hz}, 1 \mathrm{H}), 7.66-7.60(\mathrm{~m}, 1 \mathrm{H}), 7.60-7.52(\mathrm{~m}, 4 \mathrm{H}), 7.49-7.40(\mathrm{~m}$, 3 H). ${ }^{13} \mathbf{C}$ NMR (125 MHz, $\left.\mathbf{C D C l}_{3}\right) \delta 140.9,138.4,132.5,130.5,129.9,128.9,128.7,128.4$, 128.0, 127.81, 127.76, 126.6, 125.3. $\underline{\mathbf{M S}}=248.1(\mathrm{EI}) . \underline{\mathrm{HRMS}}$ calcd for $\mathrm{C}_{17} \mathrm{H}_{12} \mathrm{O}_{2} \mathrm{Na}\left(\mathrm{M}^{+}+\mathrm{Na}\right)$ 271.0735 found 271.0738 .

$N$-((1-Phenylnaphthalen-2-yl)methyl)aniline (4b, Imine reduction, Product B)
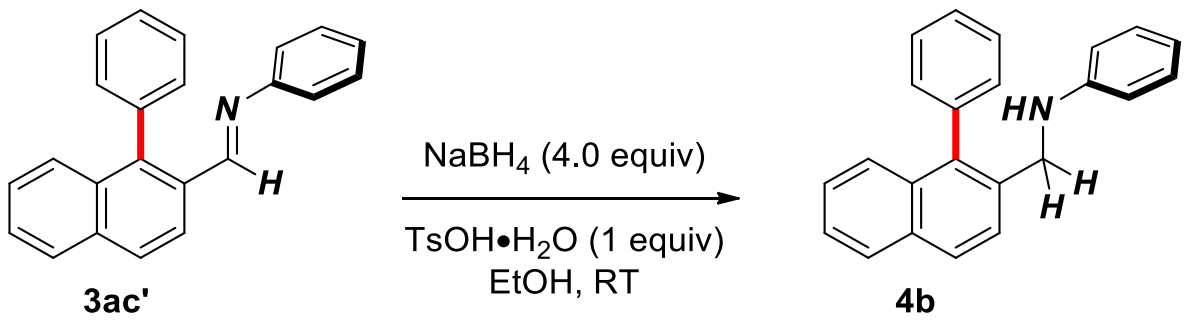

An oven-dried vial equipped with a stir bar was charged with $(E)-N-((1-$ phenylnaphthalen-2yl)methylene)aniline (3ac', $23.2 \mathrm{mg}, 0.1 \mathrm{mmol}$ ), $\mathrm{NaBH}_{4}(15.1 \mathrm{mg}, 0.4 \mathrm{mmol}), \mathrm{TsOH} \bullet \mathrm{H}_{2} \mathrm{O}(19.0$ $\mathrm{mg}, 0.1 \mathrm{mmol})$ and $\mathrm{EtOH}(2.0 \mathrm{~mL})$, and the resulting reaction mixture was stirred at room temperature for $2 \mathrm{~h}$. After the indicated time, the reaction mixture was diluted with $\mathrm{H}_{2} \mathrm{O}(10 \mathrm{~mL})$, and extracted with diethyl ether $(3 \times 10 \mathrm{~mL})$. The organic layers were combined, dried over 
$\mathrm{Na}_{2} \mathrm{SO}_{4}$, filtered and concentrated. Purification by chromatography on silica gel afforded the title product. Yield $81 \%$ (25.0 mg). White solid. $\left.\underline{\mathbf{M p}}=91-92{ }^{\circ} \mathrm{C} . \underline{{ }^{1} \mathbf{H ~ N M R ~ ( 5 0 0 ~ M H z , ~ C D C l}} \mathbf{~}\right) \delta 8.13$ $(\mathrm{d}, J=8.0 \mathrm{~Hz}, 1 \mathrm{H}), 7.97-7.92(\mathrm{~m}, 1 \mathrm{H}), 7.90(\mathrm{~d}, J=8.5 \mathrm{~Hz}, 1 \mathrm{H}), 7.56(\mathrm{dt}, J=7.5,5.9 \mathrm{~Hz}, 2 \mathrm{H})$, $7.51(\mathrm{~d}, J=8.2 \mathrm{~Hz}, 3 \mathrm{H}), 7.43(\mathrm{t}, J=7.3 \mathrm{~Hz}, 2 \mathrm{H}), 7.41-7.36(\mathrm{~m}, 1 \mathrm{H}), 7.23(\mathrm{t}, J=7.8 \mathrm{~Hz}, 2 \mathrm{H})$, $6.77(\mathrm{t}, J=7.3 \mathrm{~Hz}, 1 \mathrm{H}), 6.66(\mathrm{~d}, J=7.8 \mathrm{~Hz}, 2 \mathrm{H}), 4.58(\mathrm{~s}, 2 \mathrm{H}), 3.94$ (brs, $1 \mathrm{H}) . \underline{{ }^{13} \mathbf{C ~ N M R}(\mathbf{1 2 5}}$ $\underline{\left.\text { MHz, CDCl}_{3}\right)} \delta 148.2,141.6,140.7,133.3,132.6,130.8,129.5,129.5,128.8,128.6,128.5$, $128.4,127.5,127.3,126.1,124.8,117.6,112.8,43.2$. $\underline{\mathbf{M S}}=309.1$ (EI). $\underline{\text { HRMS }}$ calcd for $\mathrm{C}_{23} \mathrm{H}_{19} \mathrm{NNa}\left(\mathrm{M}^{+}+\mathrm{Na}\right) 332.1415$ found 332.1415 .

$N$-(1-(1-Phenylnaphthalen-2-yl)pentyl)aniline (4c, Nucleophilic addition, Product C)

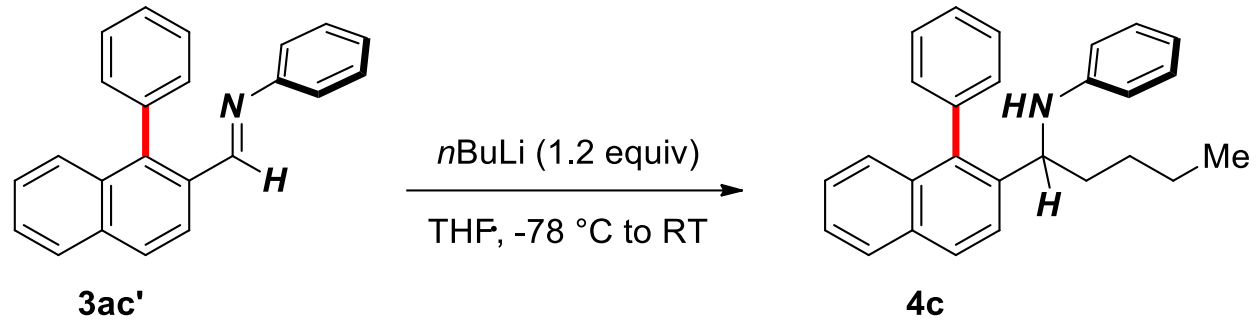

An oven-dried vial equipped with a stir bar was charged with $(E)-N-((1-$ phenylnaphthalen-2yl)methylene)aniline (3ac', $23.2 \mathrm{mg}, 0.1 \mathrm{mmol})$, anhydrous THF $(0.5 \mathrm{~mL})$, cooled to $-78^{\circ} \mathrm{C}$, and $n \operatorname{BuLi}(2.5 \mathrm{M}$ in hexanes, $0.12 \mathrm{mmol})$ was added dropwise at $-78{ }^{\circ} \mathrm{C}$. After the addition was complete, the reaction mixture was slowly warmed up to room temperature and stirred at room temperature for $2 \mathrm{~h}$. After the indicated time, the reaction mixture was diluted with $\mathrm{H}_{2} \mathrm{O}(10 \mathrm{~mL})$, and extracted with diethyl ether $(3 \times 10 \mathrm{~mL})$. The organic layers were combined, dried over $\mathrm{Na}_{2} \mathrm{SO}_{4}$, filtered and concentrated. Purification by chromatography on silica gel afforded the title

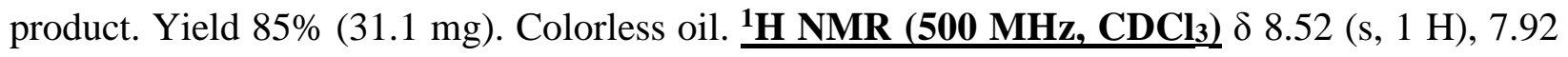
$(\mathrm{d}, J=8.0 \mathrm{~Hz}, 1 \mathrm{H}), 7.76(\mathrm{~d}, J=8.3 \mathrm{~Hz}, 1 \mathrm{H}), 7.63-7.59(\mathrm{~m}, 1 \mathrm{H}), 7.54(\mathrm{t}, J=7.3 \mathrm{~Hz}, 1 \mathrm{H}), 7.40$ (brs, 3 H), 7.27 (brs, 3 H), 7.01 (brs, 2 H), 6.58 (t, $J=6.6 \mathrm{~Hz}, 1 \mathrm{H}), 6.27$ (brs, $2 \mathrm{H}), 5.13$ (d, $J=$ $10.5 \mathrm{~Hz}, 1 \mathrm{H}), 4.15$ (brs, $1 \mathrm{H}), 2.18$ (s, $2 \mathrm{H}), 1.49-1.02$ (m, $4 \mathrm{H}), 0.85$ (s, $3 \mathrm{H}) . \underline{{ }^{\mathbf{1 3}} \mathbf{C} \text { NMR (125 }}$ $\underline{\left.\mathbf{M H z}_{2} \mathbf{C D C l}_{3}\right)} \delta 147.4,136.4,134.1,131.5,129.5,129.4,129.1,128.1,127.3,127.2,126.4$, 125.6, 116.9, 113.1, 100.2, 77.4, 29.7, 22.7, 14.2. $\underline{\mathbf{M S}}=365.2$ (EI). $\underline{\mathbf{H R M S}}$ calcd for $\mathrm{C}_{27} \mathrm{H}_{27} \mathrm{NNa}$ $\left(\mathrm{M}^{+}+\mathrm{Na}\right)$ 388.2041 found 388.2044.

$N$-(Phenyl(1-phenylnaphthalen-2-yl)methyl)aniline (4d, Imine arylation, Product D) 


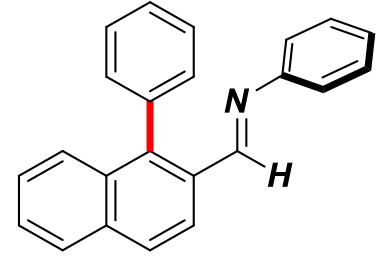

$3 \mathrm{ac}^{\prime}$

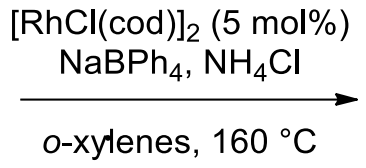

o-xylenes, $160^{\circ} \mathrm{C}$

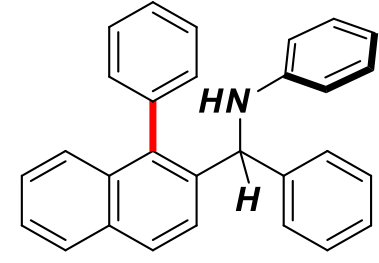

4d

Previously published procedure was followed. ${ }^{10}$ An oven-dried vial equipped with a stir bar was charged with (E)-N-((1-phenylnaphthalen-2-yl)methylene)aniline (3ac', $23.2 \mathrm{mg}, 0.1 \mathrm{mmol})$, $[\mathrm{RhCl}(\mathrm{cod})]_{2}(2.5 \mathrm{mg}, 5 \mathrm{~mol} \%), \mathrm{NaBPh}_{4}(34.2 \mathrm{mg}, 0.1 \mathrm{mmol}), \mathrm{NH}_{4} \mathrm{Cl}(5.4 \mathrm{mg}, 0.1 \mathrm{mmol})$ and $o-$ xylenes $(0.20 \mathrm{M})$, and the reaction mixture was stirred at $160{ }^{\circ} \mathrm{C}$ for $24 \mathrm{~h}$. After the indicated time, the reaction mixture was purified directly by chromatography on silica gel to afford the title product. Yield 70\% (27.0 mg). White solid. $\underline{\mathbf{M p}}=98-99{ }^{\circ} \mathrm{C} . \underline{\mathbf{1}} \mathbf{\mathrm { H }} \mathbf{\mathrm { NMR }}(\mathbf{5 0 0} \mathbf{\mathrm { MHz }}, \mathbf{C D C l} \mathbf{3}) \delta 8.01$ $(\mathrm{d}, J=8.6 \mathrm{~Hz}, 1 \mathrm{H}), 7.90(\mathrm{~d}, J=8.1 \mathrm{~Hz}, 1 \mathrm{H}), 7.87(\mathrm{~d}, J=8.4 \mathrm{~Hz}, 1 \mathrm{H}), 7.49(\mathrm{~d}, J=8.4 \mathrm{~Hz}, 1 \mathrm{H})$, $7.44(\mathrm{t}, J=7.4 \mathrm{~Hz}, 1 \mathrm{H}), 7.42-7.30(\mathrm{~m}, 4 \mathrm{H}), 7.30-7.26(\mathrm{~m}, 3 \mathrm{H}), 7.23(\mathrm{t}, J=7.5 \mathrm{~Hz}, 3 \mathrm{H}), 7.17$ (t, $J=7.0 \mathrm{~Hz}, 1 \mathrm{H}), 7.09(\mathrm{t}, J=7.7 \mathrm{~Hz}, 2 \mathrm{H}), 6.70(\mathrm{t}, J=7.2 \mathrm{~Hz}, 1 \mathrm{H}), 6.41(\mathrm{~d}, J=7.9 \mathrm{~Hz}, 2 \mathrm{H})$,

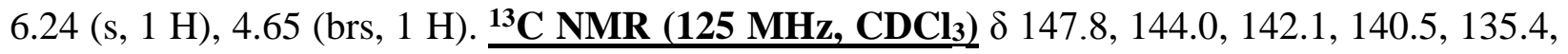
$134.5,131.0,129.3,129.2,128.50,128.47,128.3,127.5,126.7,126.59,126.55,126.5,125.7$, 117.7, 113.2, 58.3. $\underline{\mathbf{M S}}=385.1(\mathrm{EI})$. $\underline{\text { HRMS }}$ calcd for $\mathrm{C}_{29} \mathrm{H}_{23} \mathrm{NNa}\left(\mathrm{M}^{+}+\mathrm{Na}\right) 408.1728$ found 408.1729 .

\section{2-([1,1'-Biphenyl]-2-yl)-1H-indole (4e, Indole Synthesis)}

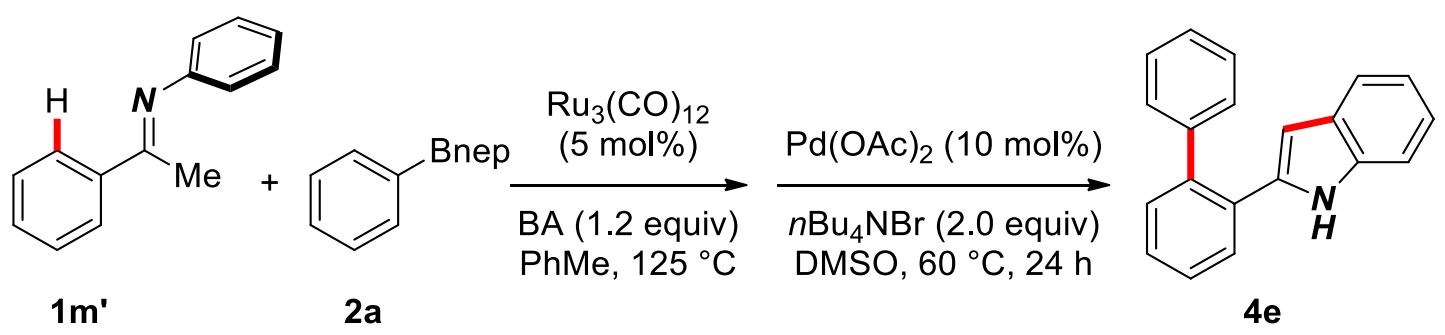

Previously published procedure was followed. ${ }^{11}$ According the general procedure A, $(E)-N-(1-$ phenylethylidene)aniline (1j, $39.0 \mathrm{mg}, 0.20 \mathrm{mmol})$ was reacted with PhBnep (2a, $38.0 \mathrm{mg}, 0.20$ $\mathrm{mmol}), \mathrm{BA}\left(35.1 \mathrm{mg}, 0.24 \mathrm{mmol}, 1.2\right.$ equiv) and $\mathrm{Ru}_{3}(\mathrm{CO})_{12}(6.4 \mathrm{mg}, 5 \mathrm{~mol} \%)$ in toluene $(1.0 \mathrm{M})$ at $125{ }^{\circ} \mathrm{C}$ for $1 \mathrm{~h}$. After the indicated time, the reaction mixture was cooled down to room temperature, $\mathrm{Pd}(\mathrm{OAc})_{2}(4.5 \mathrm{mg}, 0.02 \mathrm{mmol}, 10 \mathrm{~mol} \%), n \mathrm{Bu}_{4} \mathrm{NBr}(129.0 \mathrm{mg}, 0.4 \mathrm{mmol})$, and DMSO $(1 \mathrm{~mL})$ were sequentially added. The resulting reaction mixture was stirred at $65{ }^{\circ} \mathrm{C}$ 
under $\mathrm{O}_{2}$ for $24 \mathrm{~h}$. After the indicated time, the reaction mixture was diluted with $\mathrm{H}_{2} \mathrm{O}(10 \mathrm{~mL})$, and extracted with dichloromethane $(3 \times 10 \mathrm{~mL})$. The organic layers were combined, dried over $\mathrm{Na}_{2} \mathrm{SO}_{4}$, filtered and concentrated. Purification by chromatography on silica gel afforded the title product. Two-step yield 77\% (41.4 mg). White solid. $\underline{\mathbf{M p}}=93-94{ }^{\circ} \mathrm{C} . \underline{{ }^{1} \mathbf{H} \text { NMR (500 MHz}}$ $\left.\underline{\mathbf{C D C l}_{3}}\right) \delta 7.75(\mathrm{~d}, J=7.2 \mathrm{~Hz}, 1 \mathrm{H}), 7.59(\mathrm{~d}, J=7.2 \mathrm{~Hz}, 1 \mathrm{H}), 7.53$ (brs, $\left.1 \mathrm{H}\right), 7.48-7.40(\mathrm{~m}, 3 \mathrm{H})$,

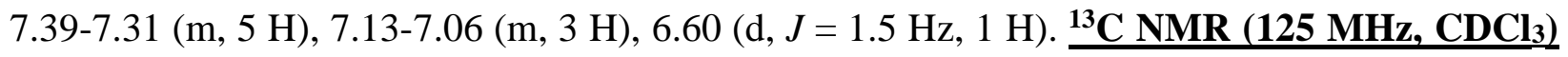
$\delta 141.3,139.8,137.9,136.5,131.3,131.2,130.1,129.5,128.7,128.5,128.1,128.0,127.6,122.1$, 120.6, 120.0, 110.9, 102.5. $\underline{\mathbf{M S}}=269.1(\mathrm{EI}) \underline{\text { HRMS }}$ calcd for $\mathrm{C}_{20} \mathrm{H}_{15} \mathrm{NNa}\left(\mathrm{M}^{+}+\mathrm{Na}\right) 292.1102$ found 292.1104 . 


\section{References}

1. Trost, B. M.; Fleming, I. Comprehensive Organic Synthesis; Pergamon Press: 1991.

2. Smith, M. B.; March, J. Advanced Organic Chemistry; Wiley: 2007.

3. Layer, R. W. Chem. Rev. 1963, 63, 489.

4. Patil, R. D.; Adimurthy, S. Asian J. Org. Chem. 2013, 2, 726.

5. Tredwell, M. J.; Gulias, M.; Gaunt-Bremeyer, N.; Johansson, C. C. C.; Collins, B. S. L.; Gaunt, M. J. Angew. Chem. Int. Ed. 2011, 50, 1076.

6. Iwai, Y.; Gligorich, K. M.; Sigman, M. S. Angew. Chem. Int. Ed. 2008, 47, 3219.

7. Zhao, Y.; Snieckus, V. J. Am. Chem. Soc. 2014, 136, 11224.

8. Sollert, C.; Devaraj, K.; Orthaber, A.; Gates, P. J.; Pilarski, L. T. Chem. Eur. J. 2015, 21, 5380.

9. Chinnagolla, R. K.; Jeganmohan, M. Org. Lett. 2012, 14, 5246.

10. Ueura, K.; Miyamura, S.; Satoh, T.; Miura, M. J. Organomet. Chem. 2006, 691, 2821.

11. Wei, Y.; Deb, I.; Yoshikai, N. J. Am. Chem. Soc. 2012, 134, 9098. 


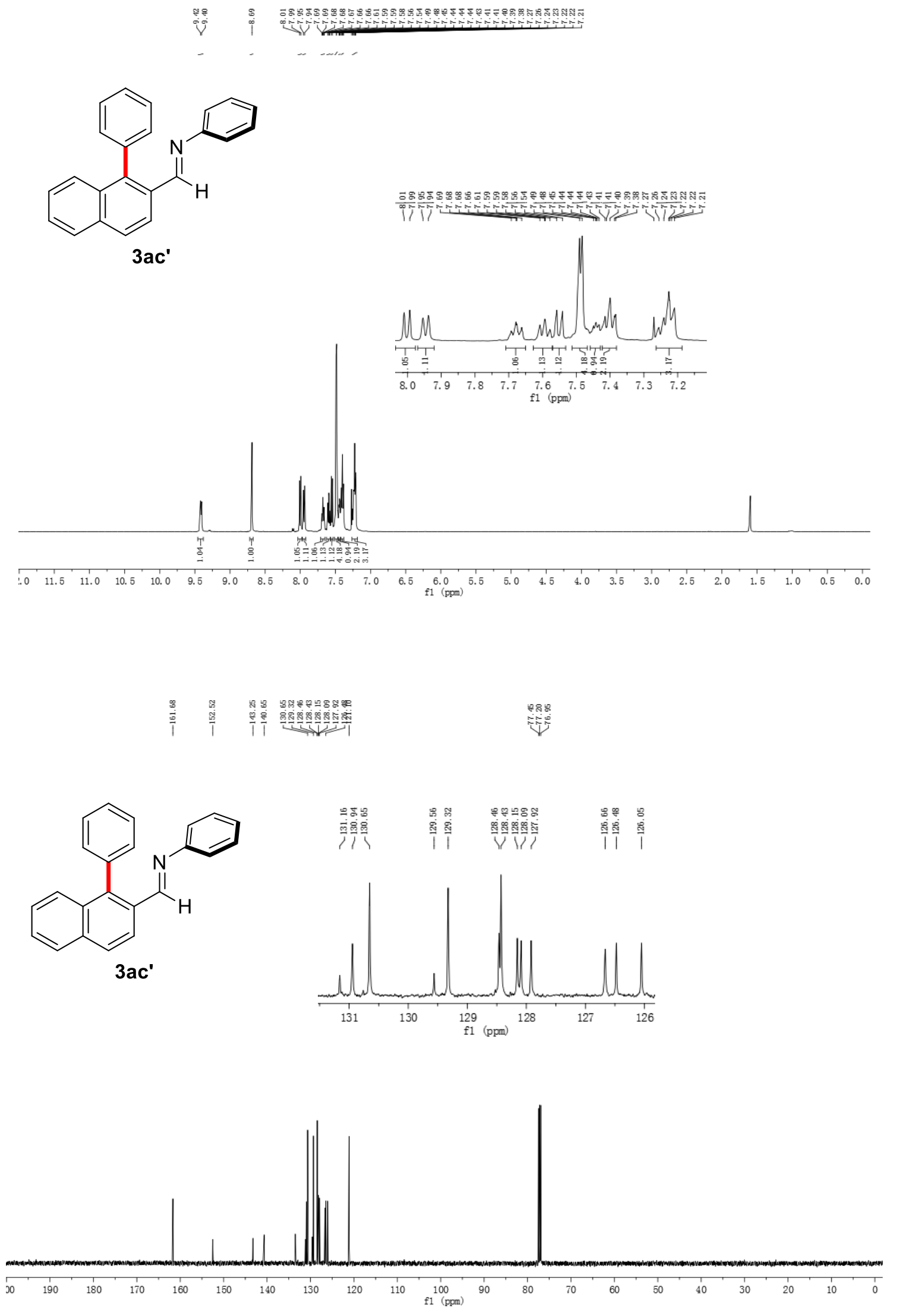

SI-34 


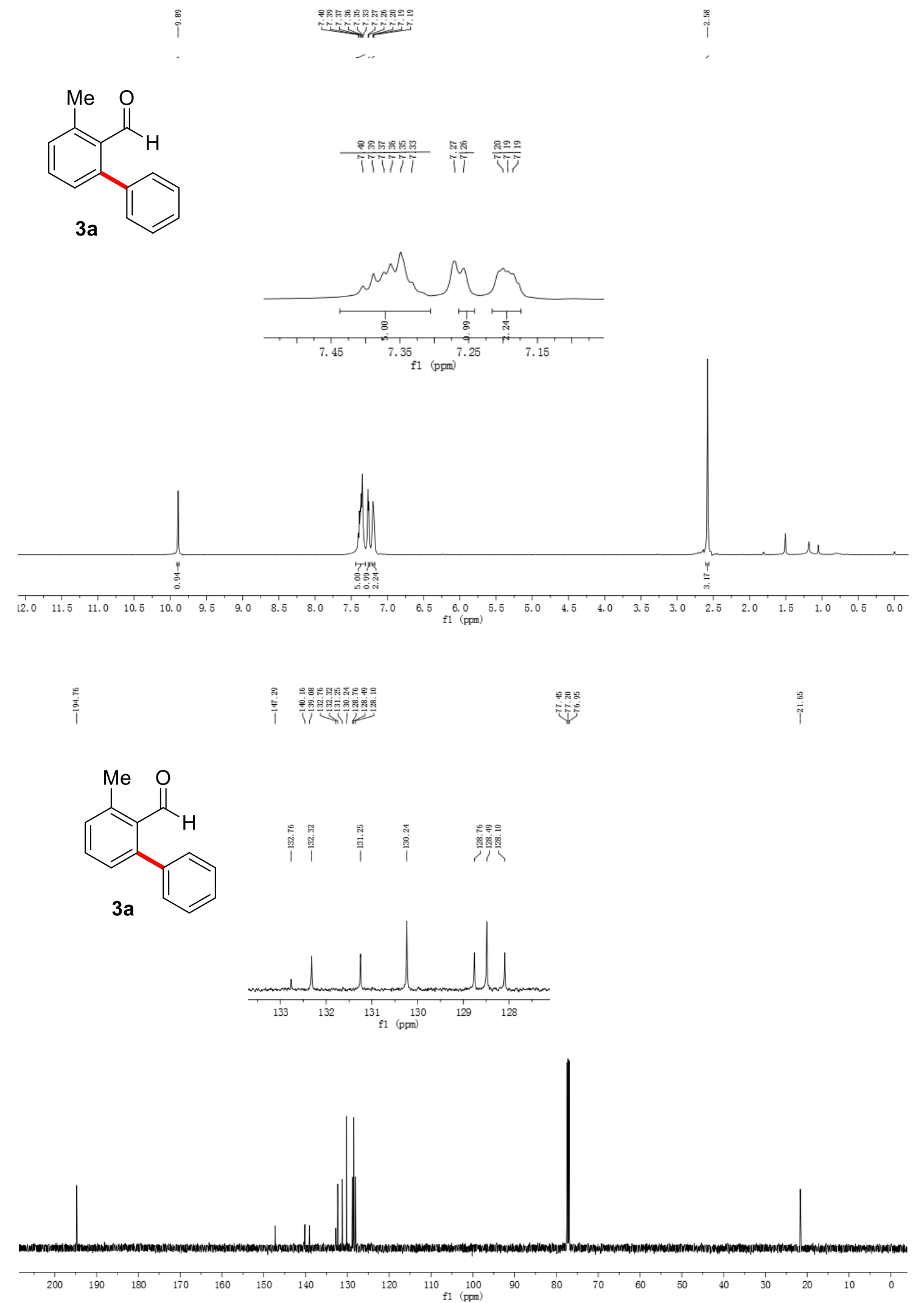

SI-35 
<smiles>Cc1ccc(-c2cccc(C)c2C=O)cc1</smiles>
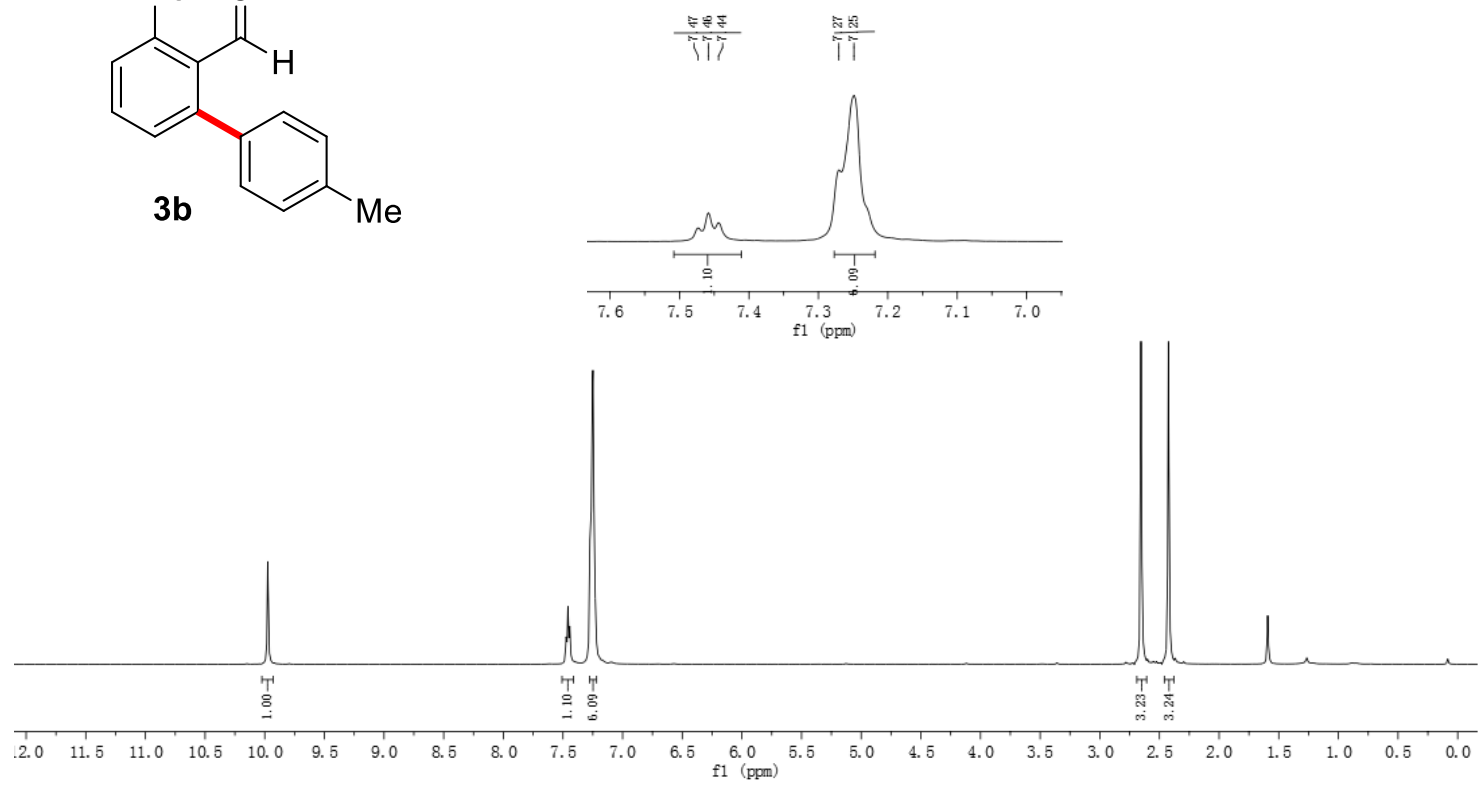

$\frac{1}{1}$

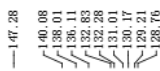

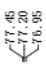<smiles>Cc1ccc(-c2cccc(C)c2C=O)cc1</smiles>

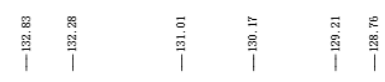

3b
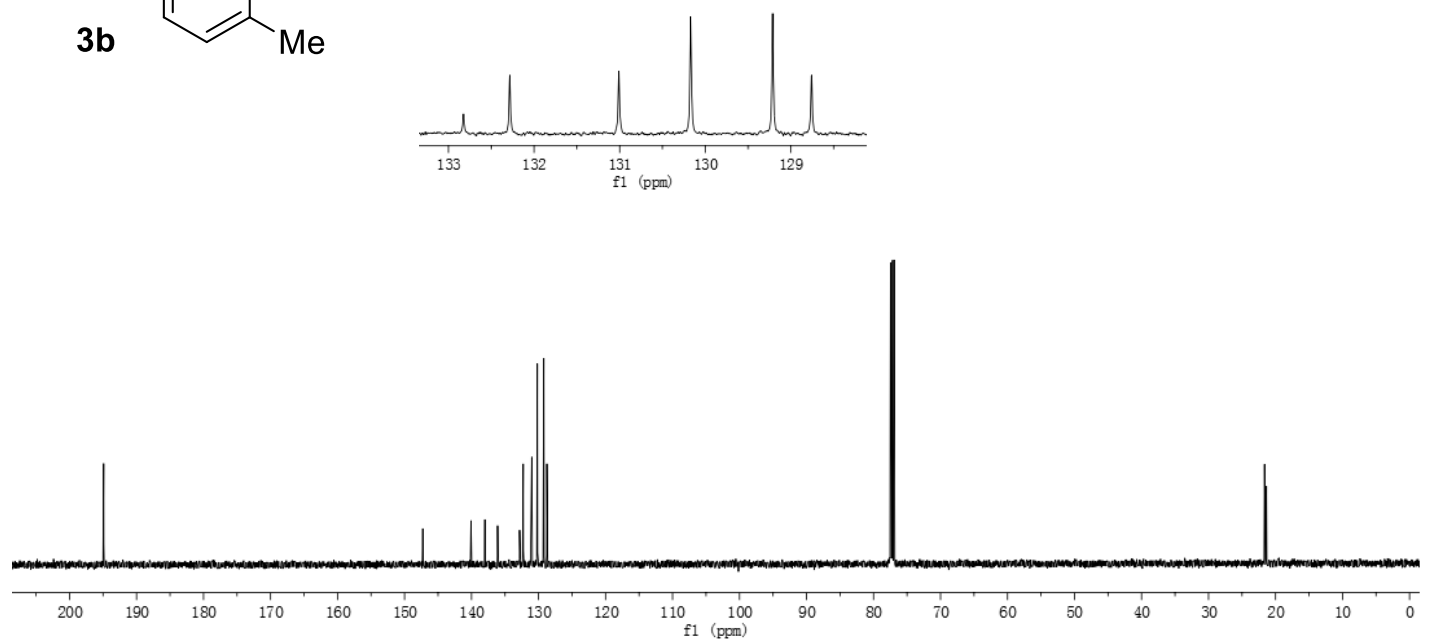

SI-36 


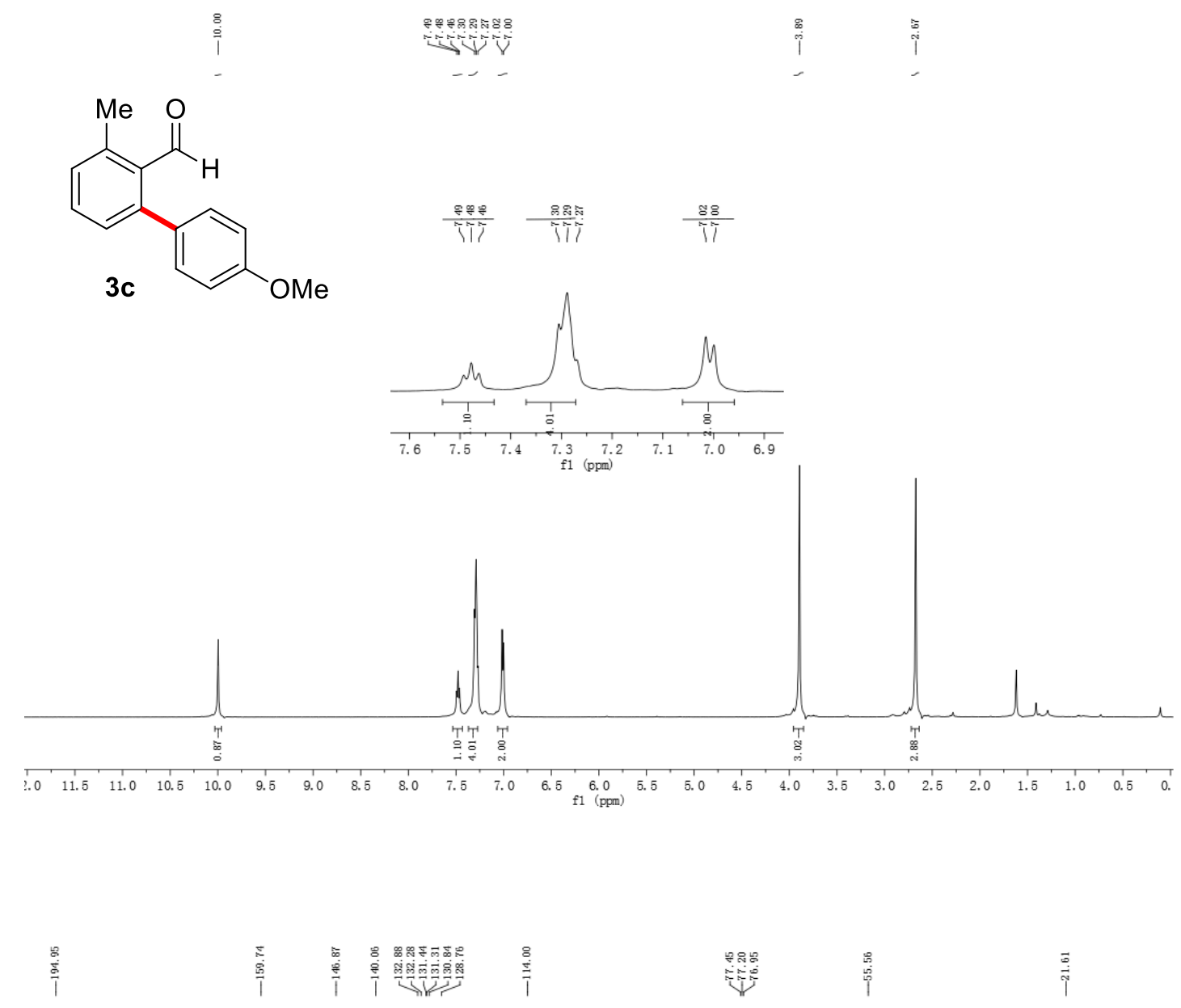<smiles>COc1ccc(-c2cccc(C)c2C=O)cc1</smiles>

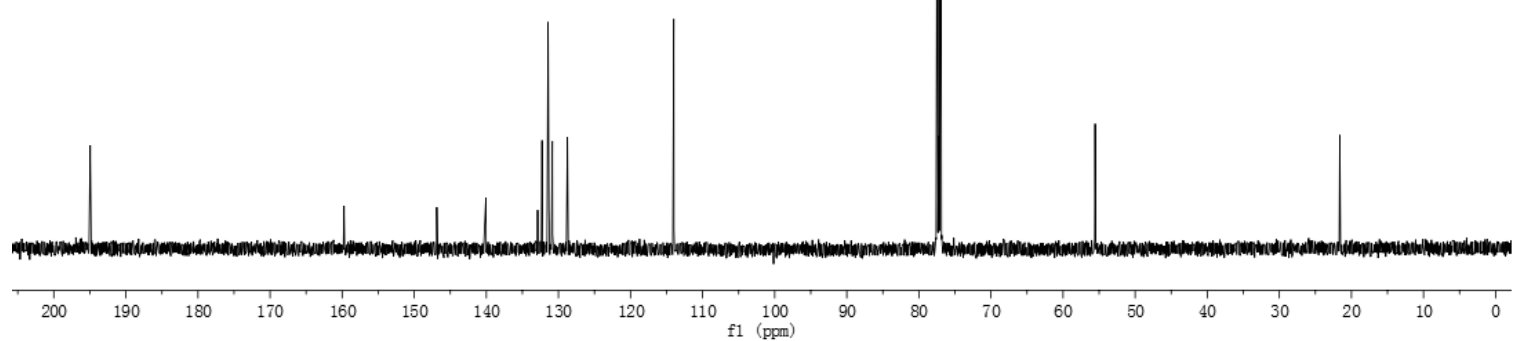

SI-37 
i

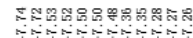

itisinitis

$\ldots$<smiles>Cc1cccc(-c2ccc(C(F)(F)F)cc2)c1C=O</smiles>

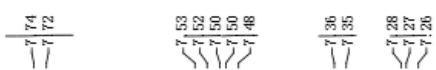
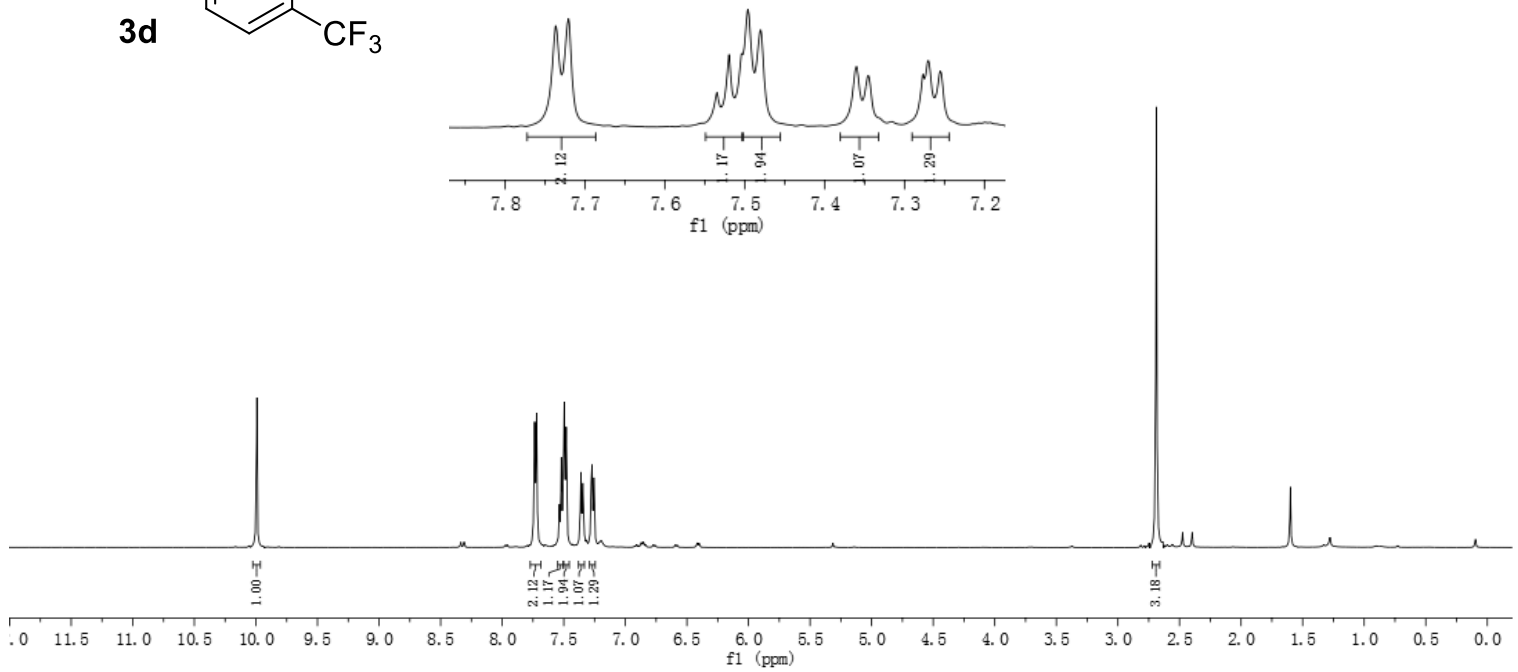

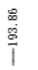

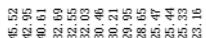

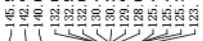

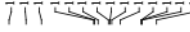

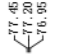

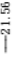<smiles>Cc1cccc(-c2ccc(C(F)(F)F)cc2)c1C=O</smiles>

\begin{tabular}{|c|c|c|c|}
\hline 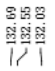 & 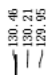 & 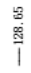 & 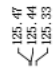 \\
\hline
\end{tabular}
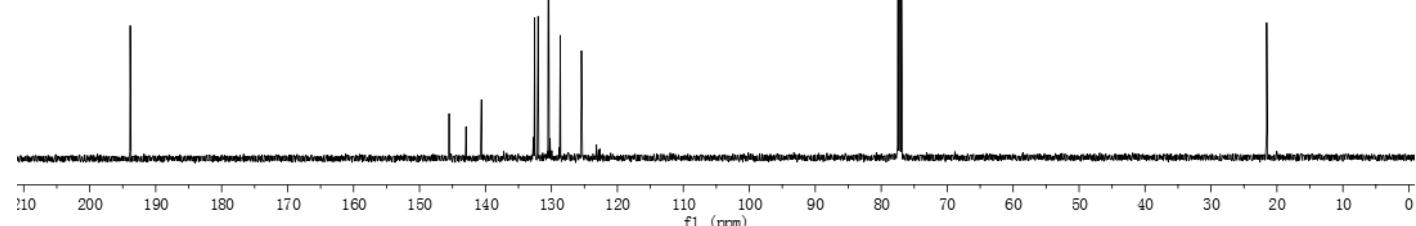

SI-38 
<smiles>Cc1cccc(-c2ccc(C(F)(F)F)cc2)c1C=O</smiles>

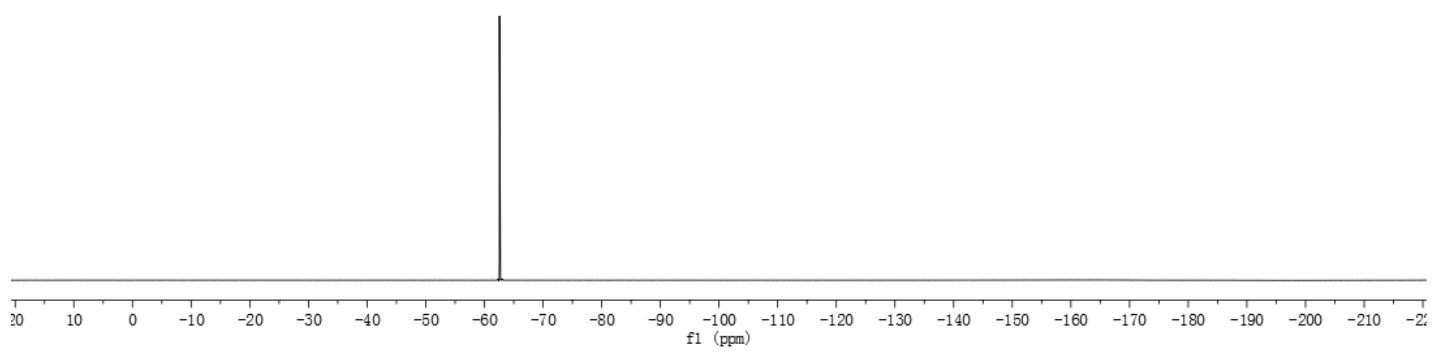


<smiles>Cc1cccc(-c2ccc(F)cc2)c1C=O</smiles>

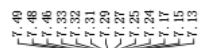

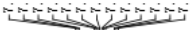

$3 \mathbf{e}$

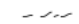

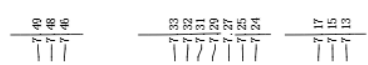
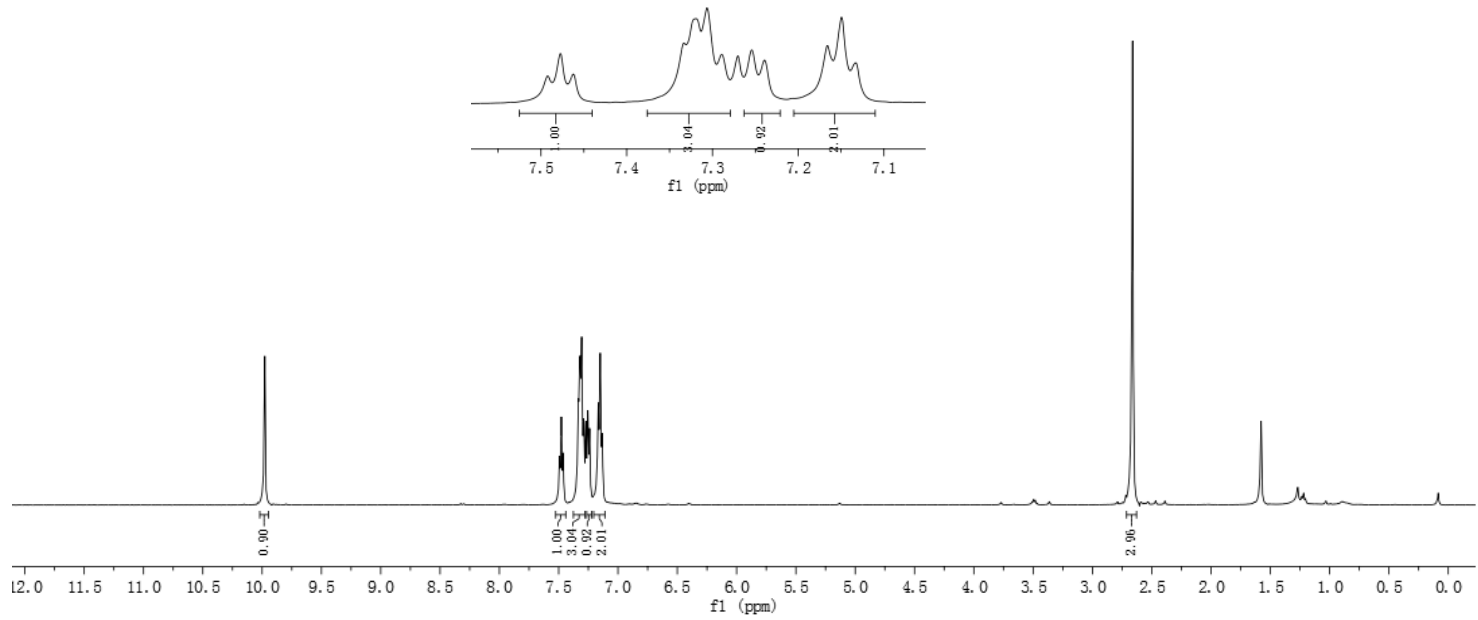

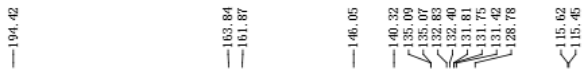

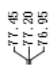<smiles>Cc1cccc(-c2ccc(F)cc2)c1C=O</smiles>

$3 e$
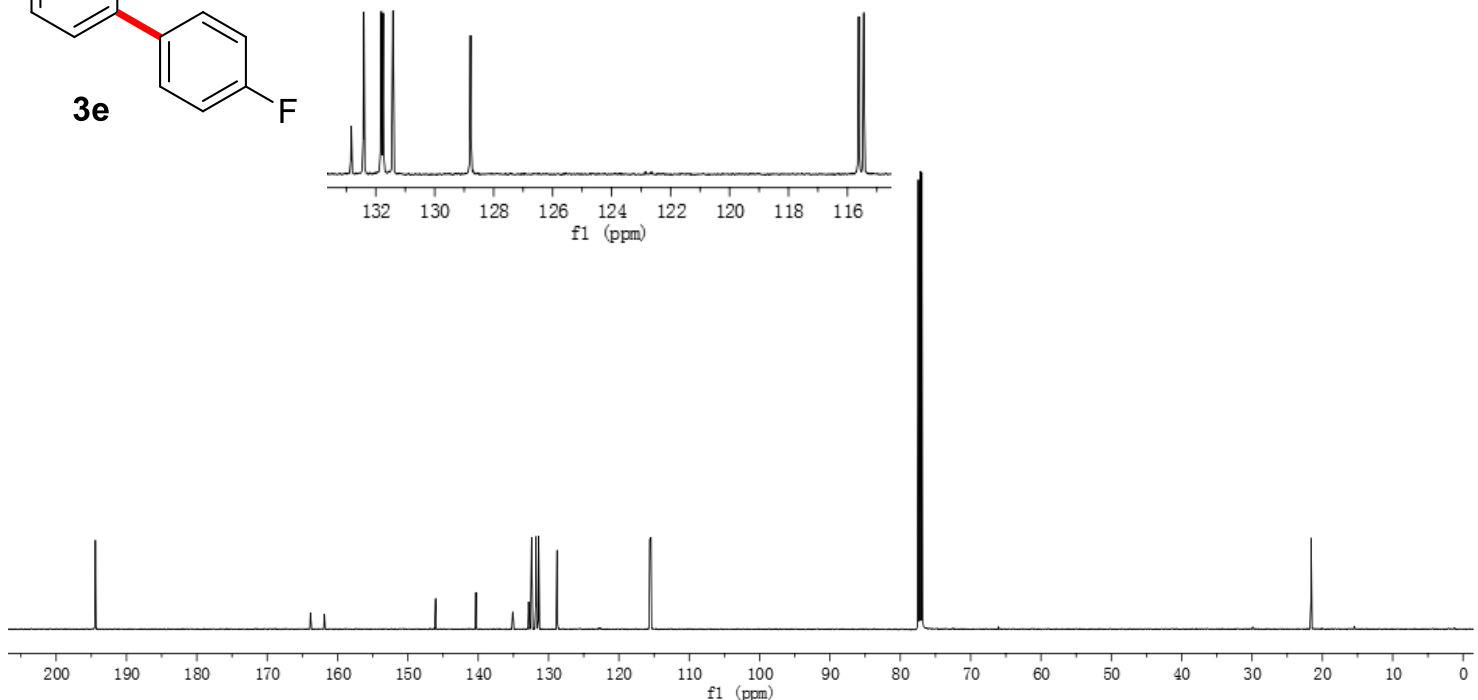

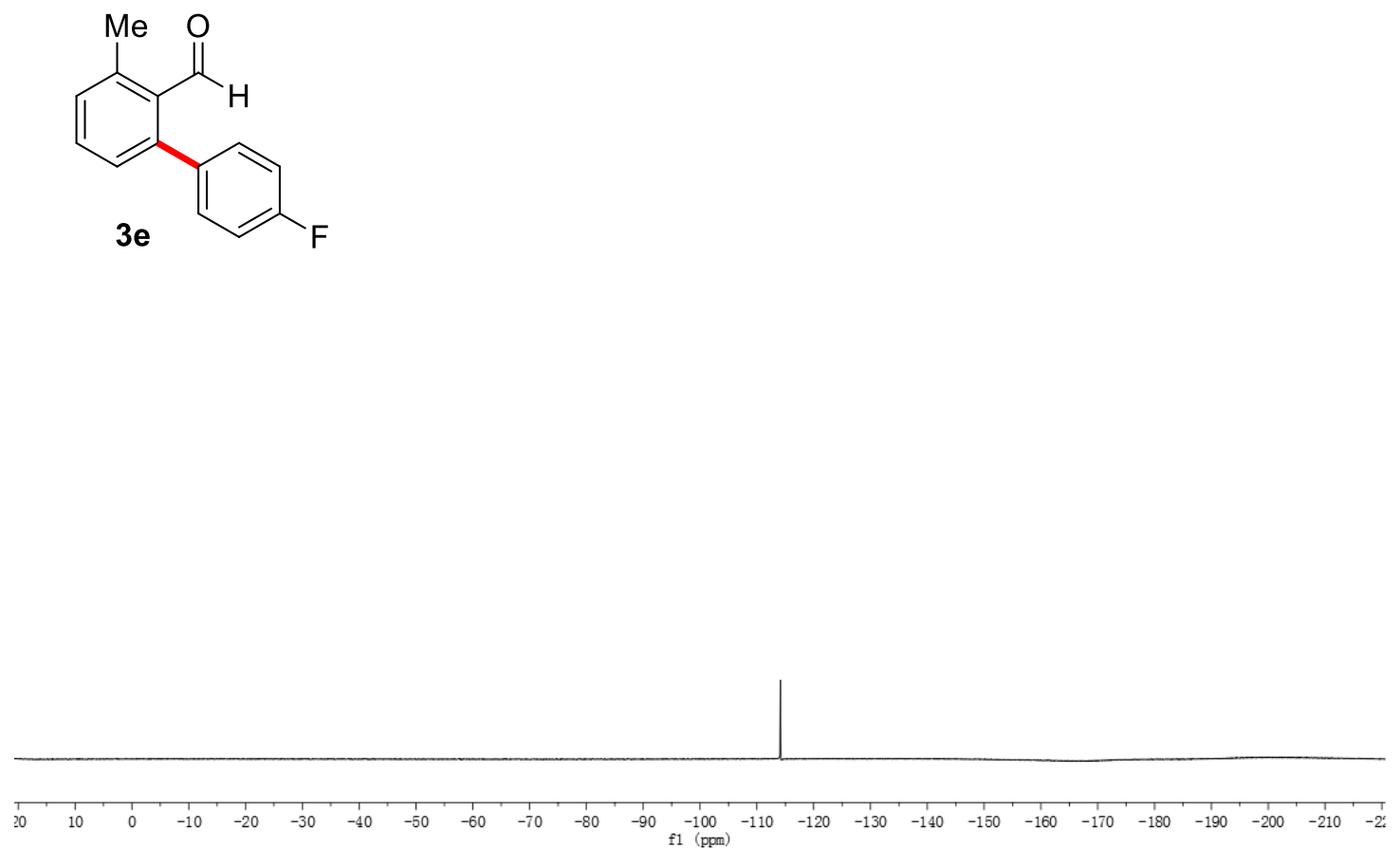


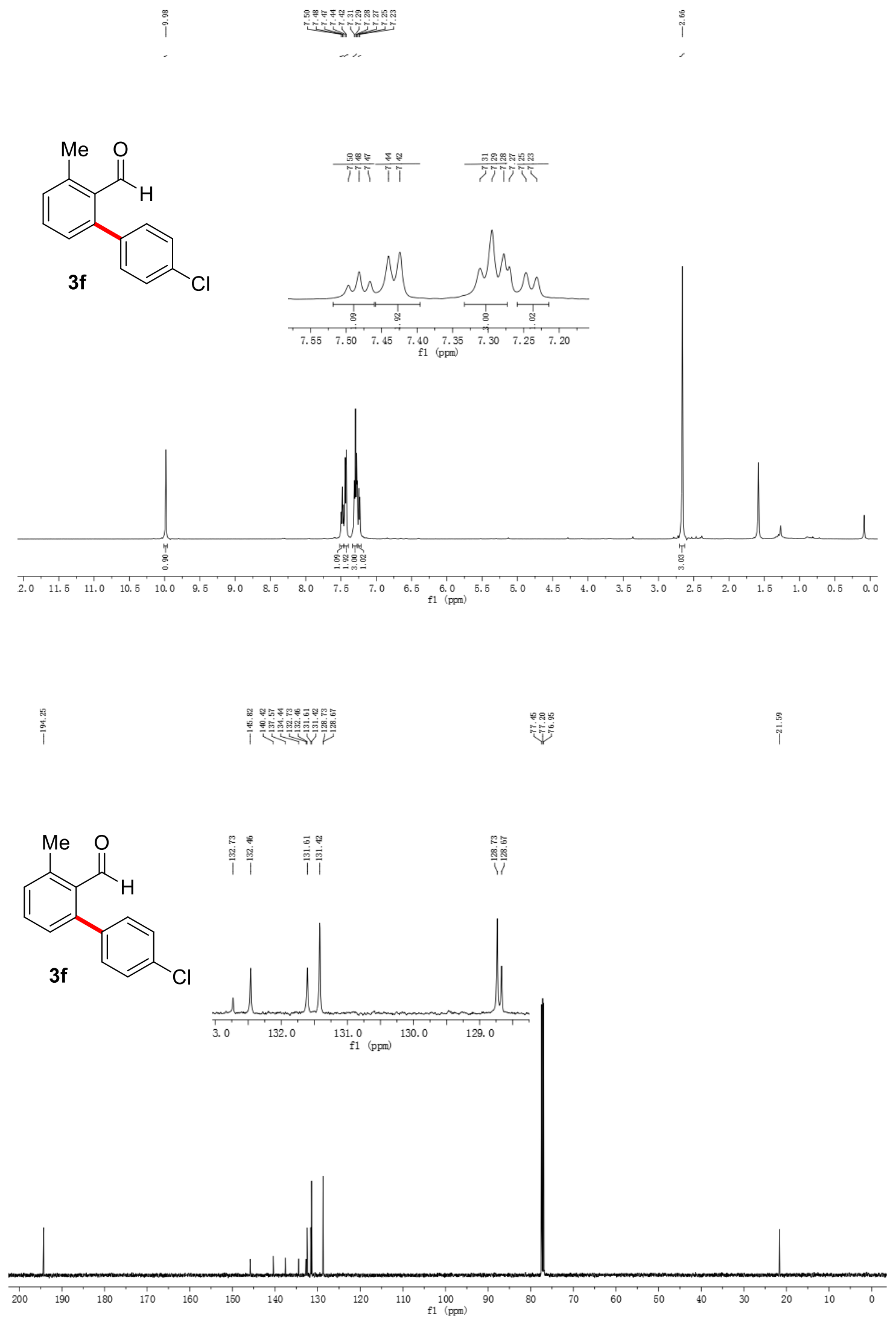



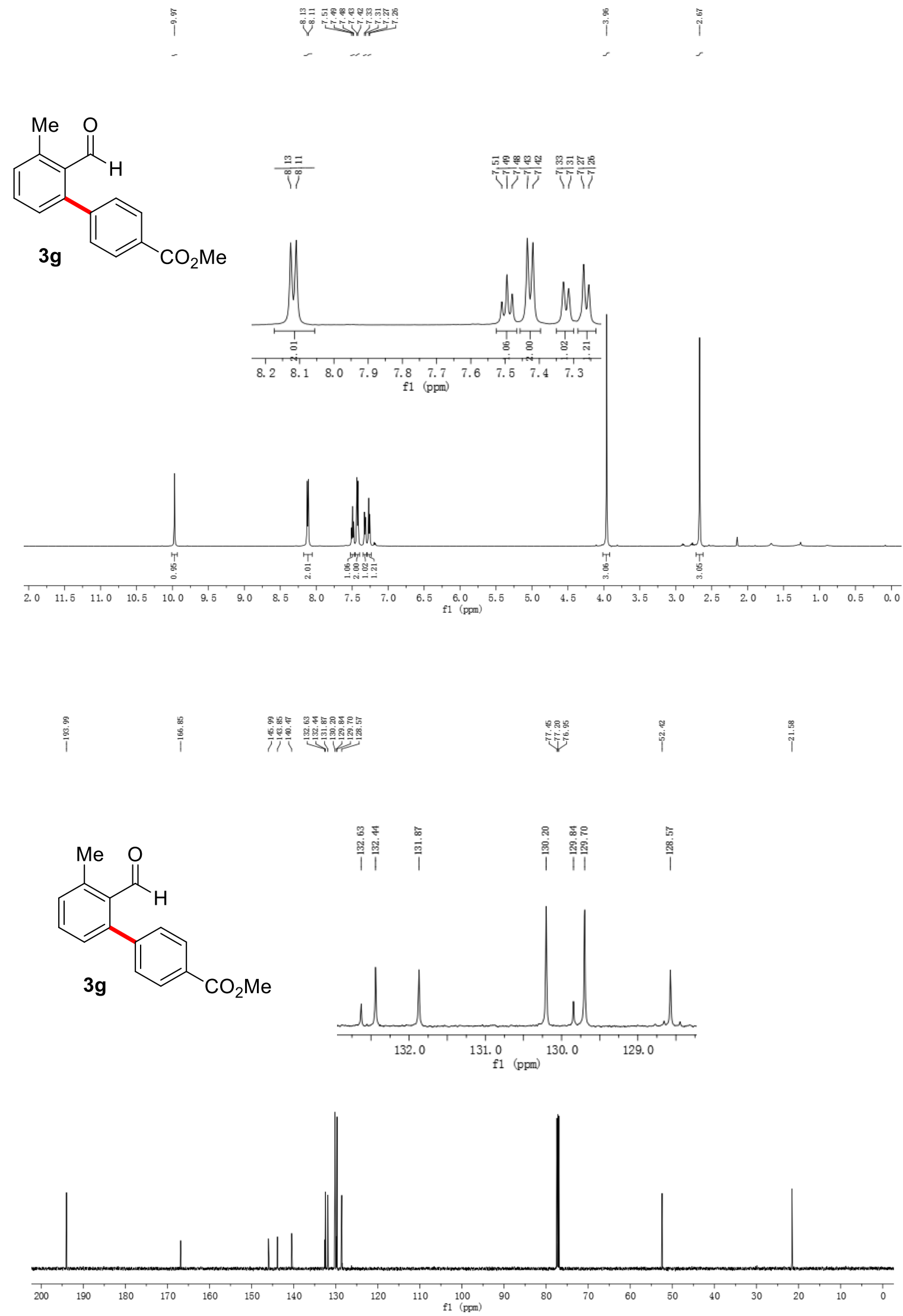

SI-43 

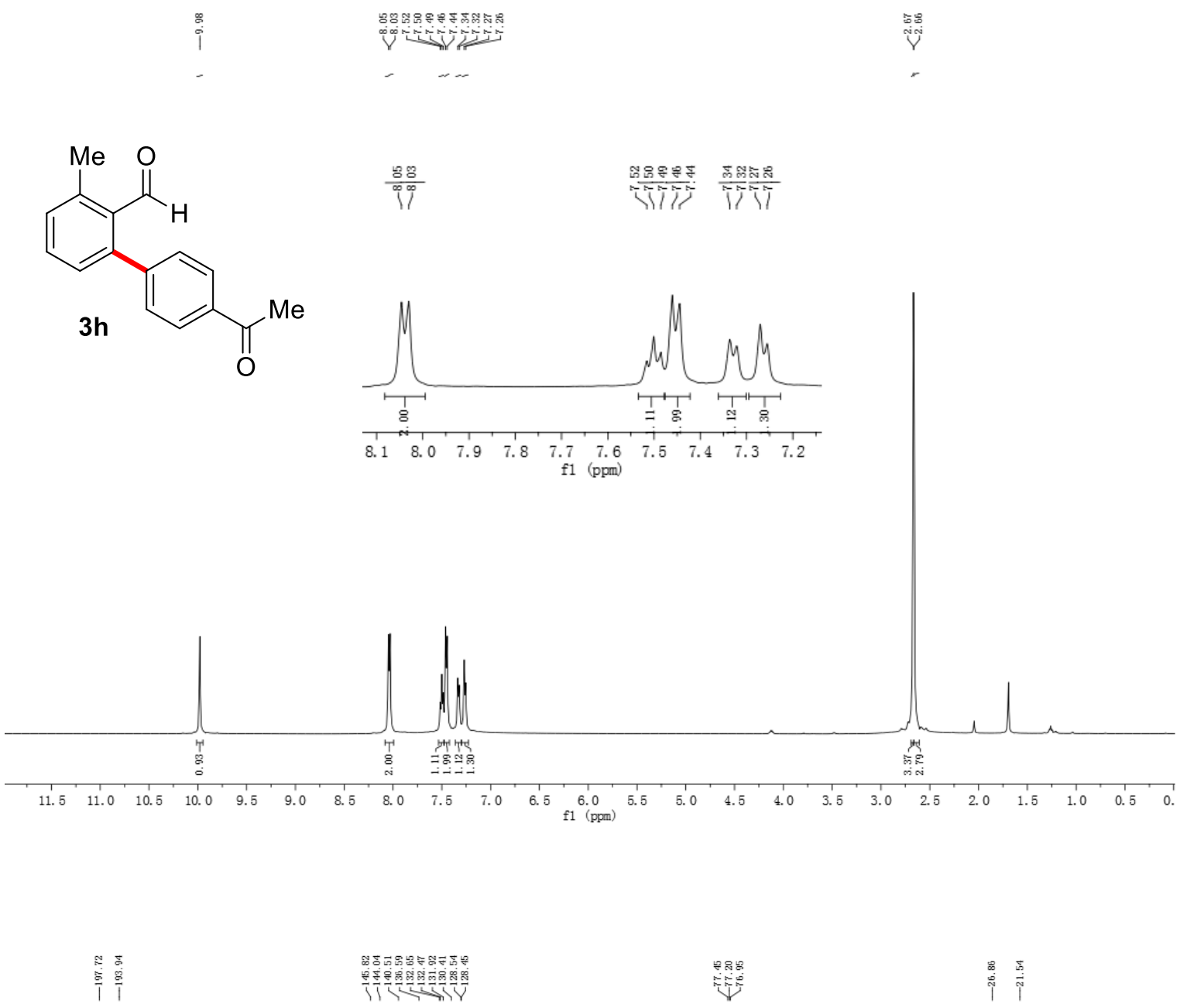<smiles>CC(=O)c1ccc(-c2cccc(C)c2C=O)cc1</smiles>
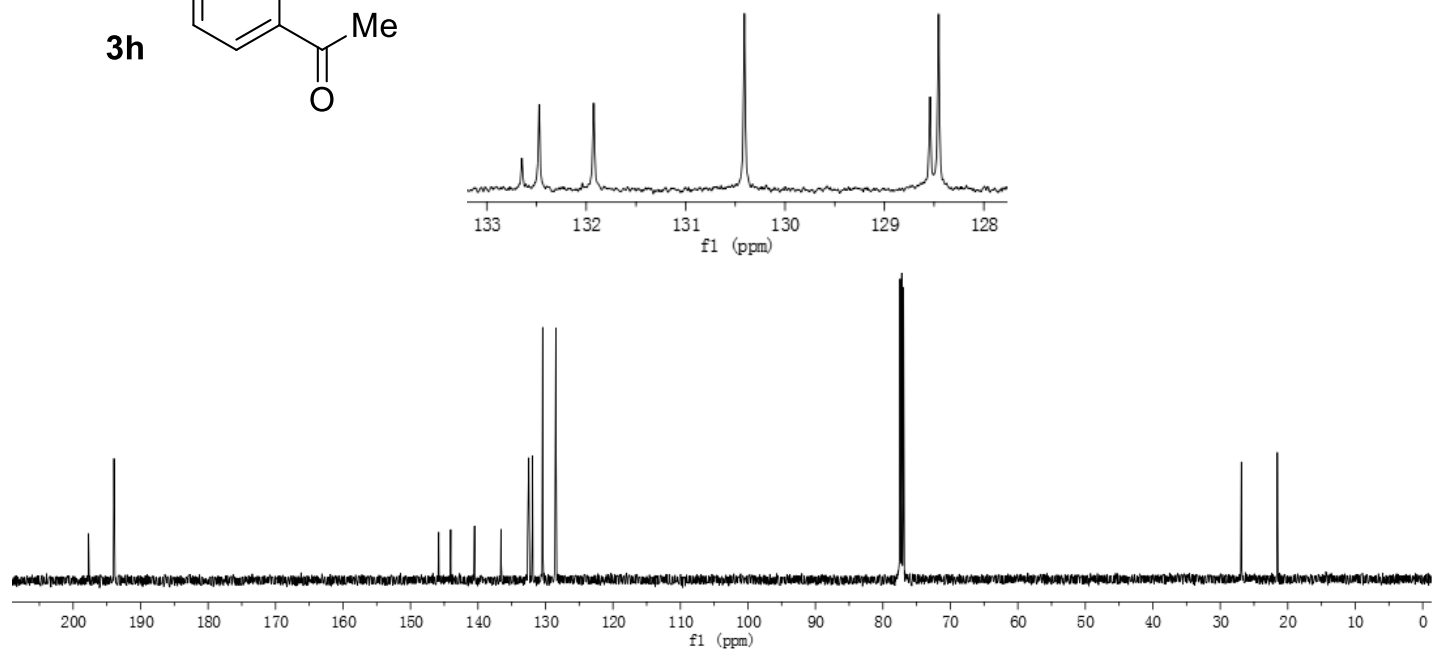

\section{SI-44}




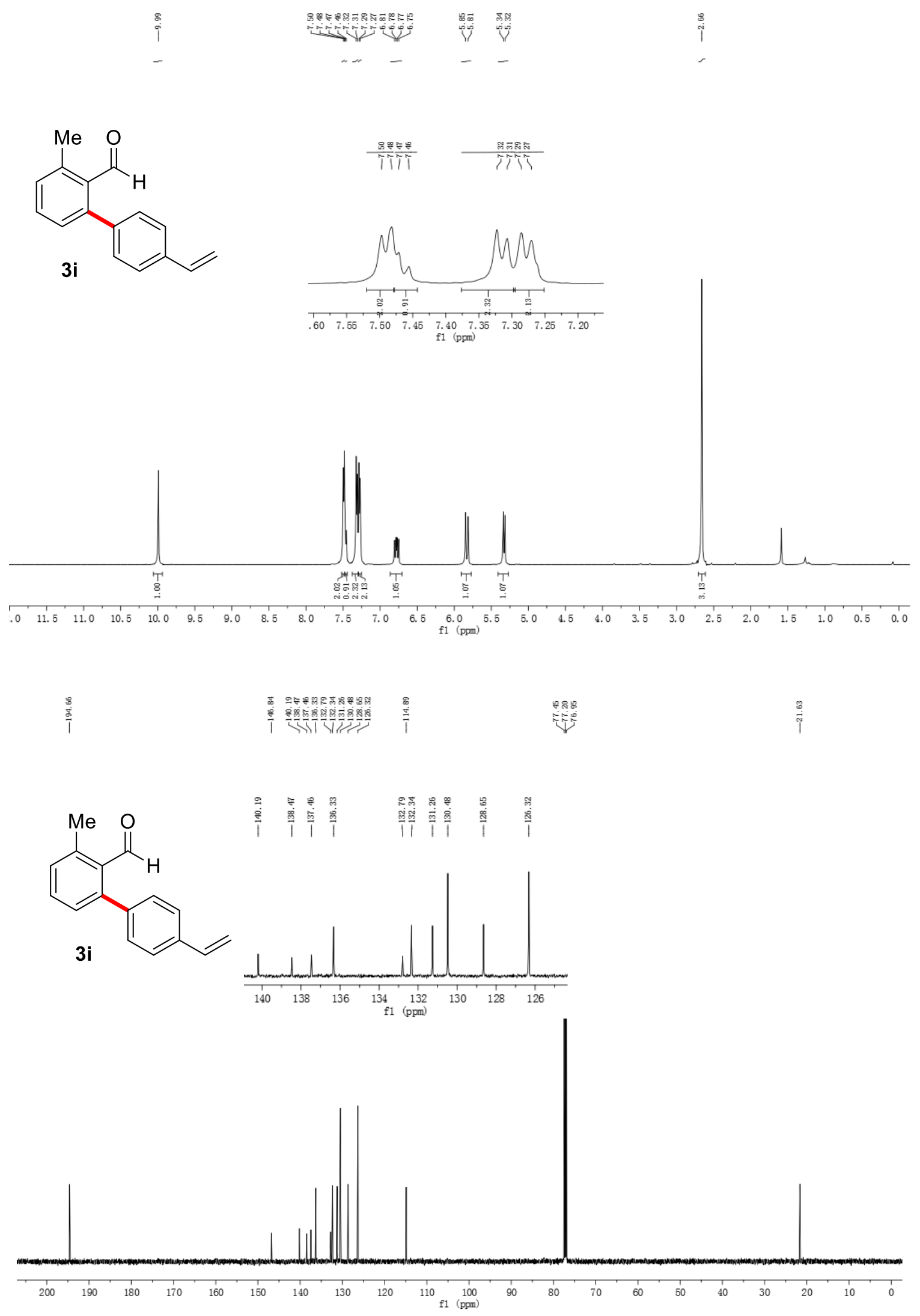




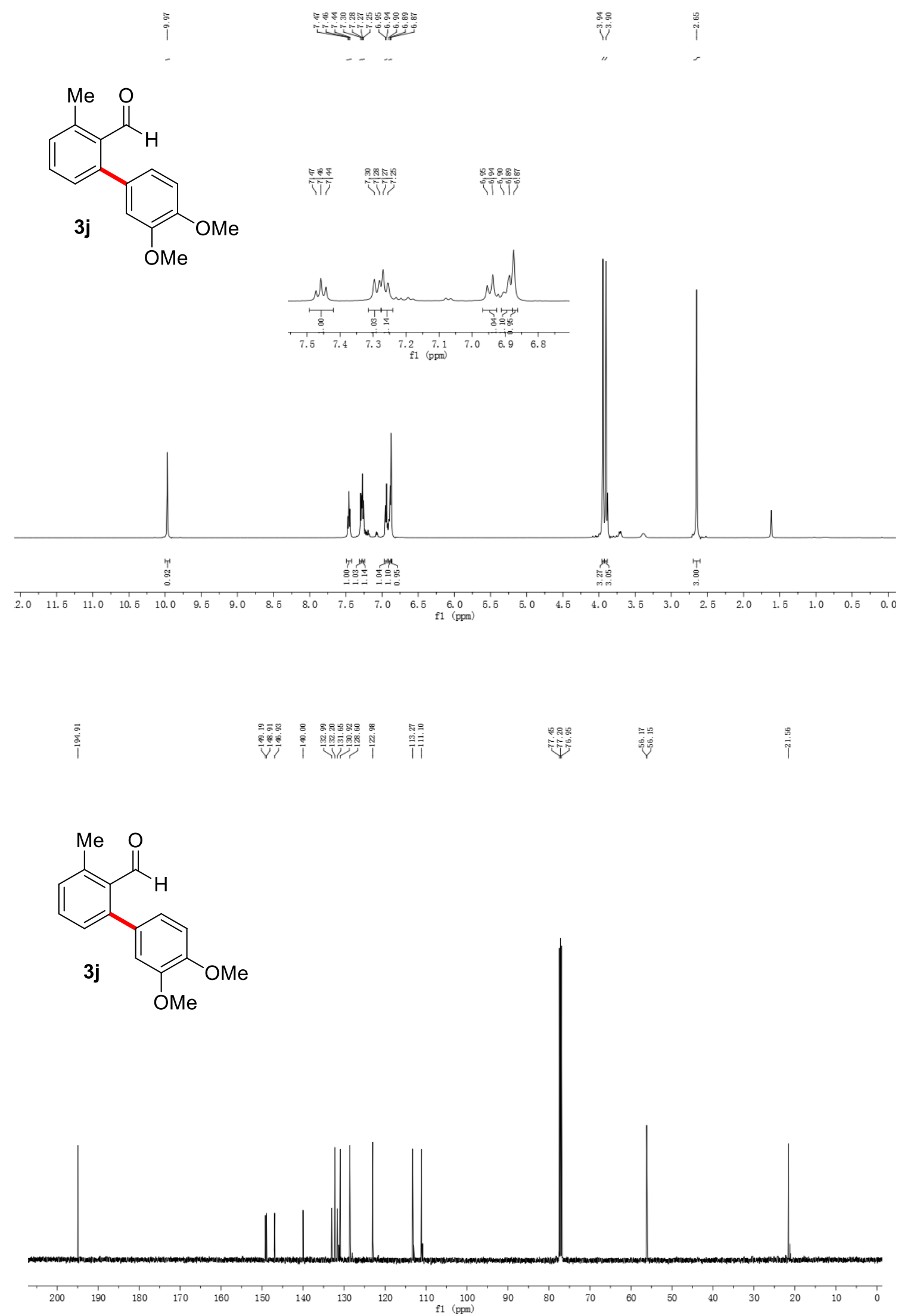

SI-46 
<smiles>Cc1cccc(-c2cc(F)cc(F)c2)c1C=O</smiles>
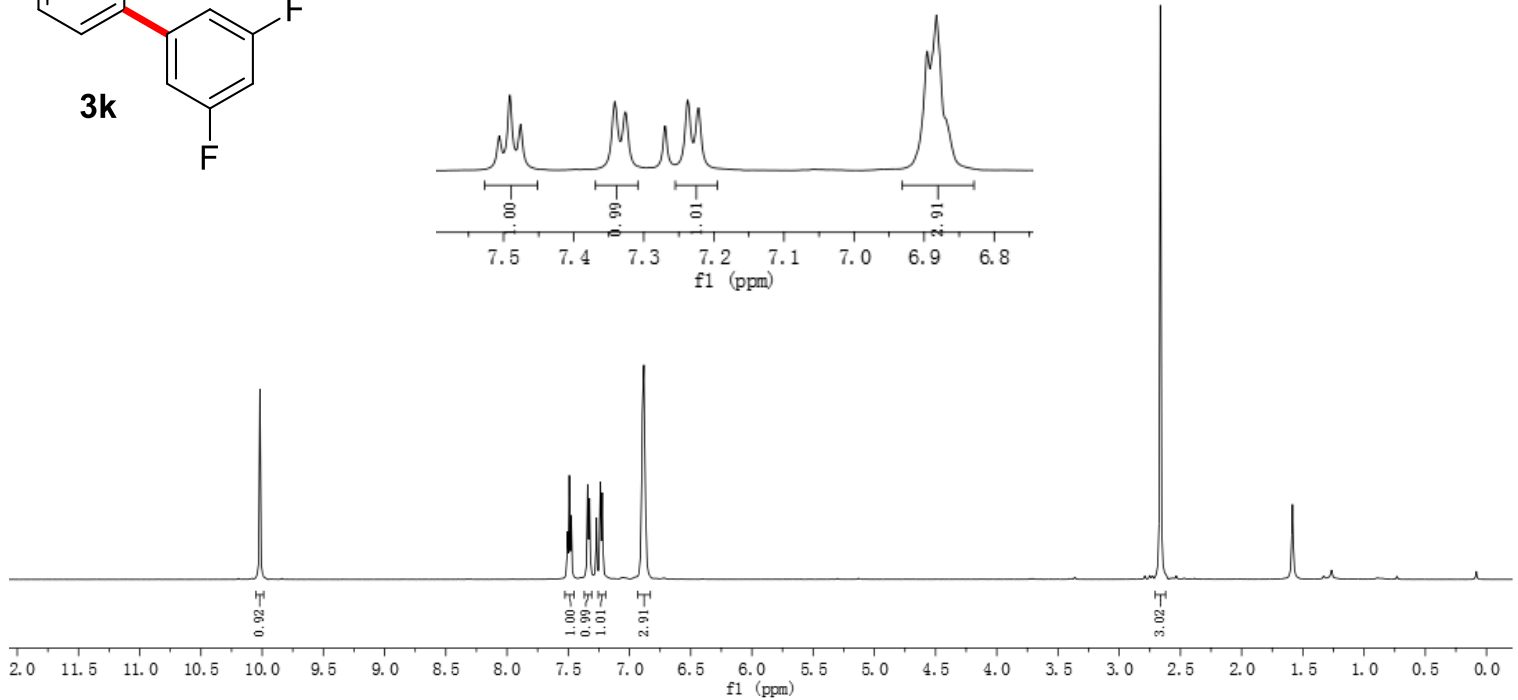

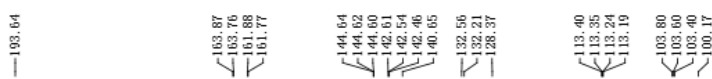

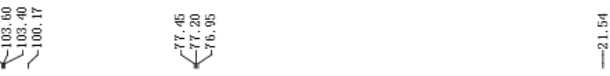<smiles>Cc1cccc(-c2cc(F)cc(F)c2)c1C=O</smiles>
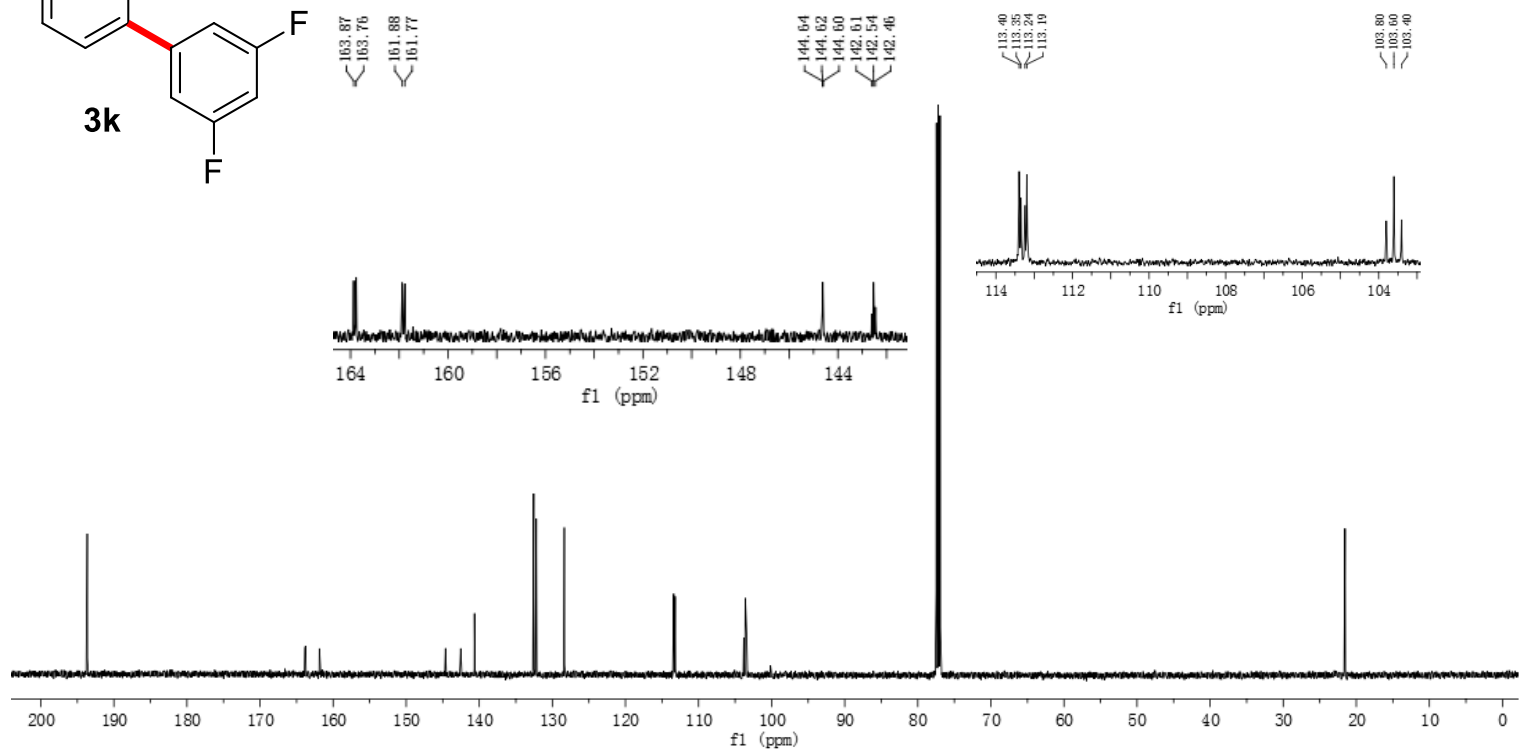
<smiles>Cc1cccc(-c2cc(F)cc(F)c2)c1C=O</smiles>

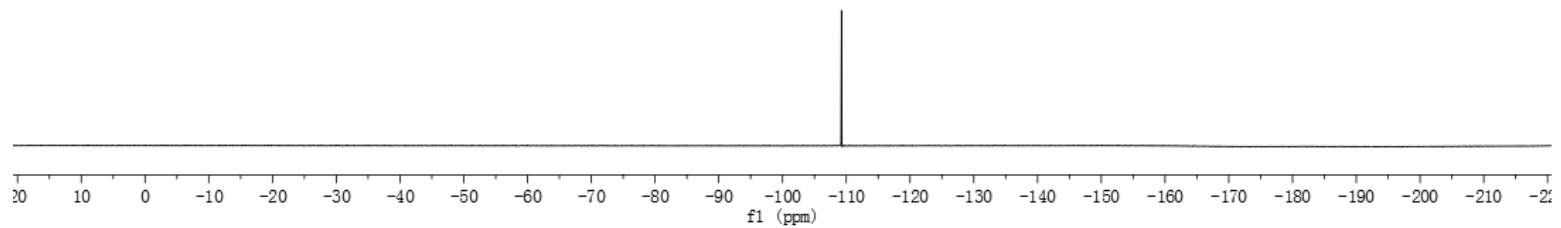




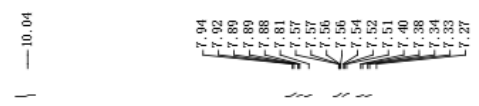<smiles>Cc1cccc(-c2ccc3ccccc3c2)c1C=O</smiles>
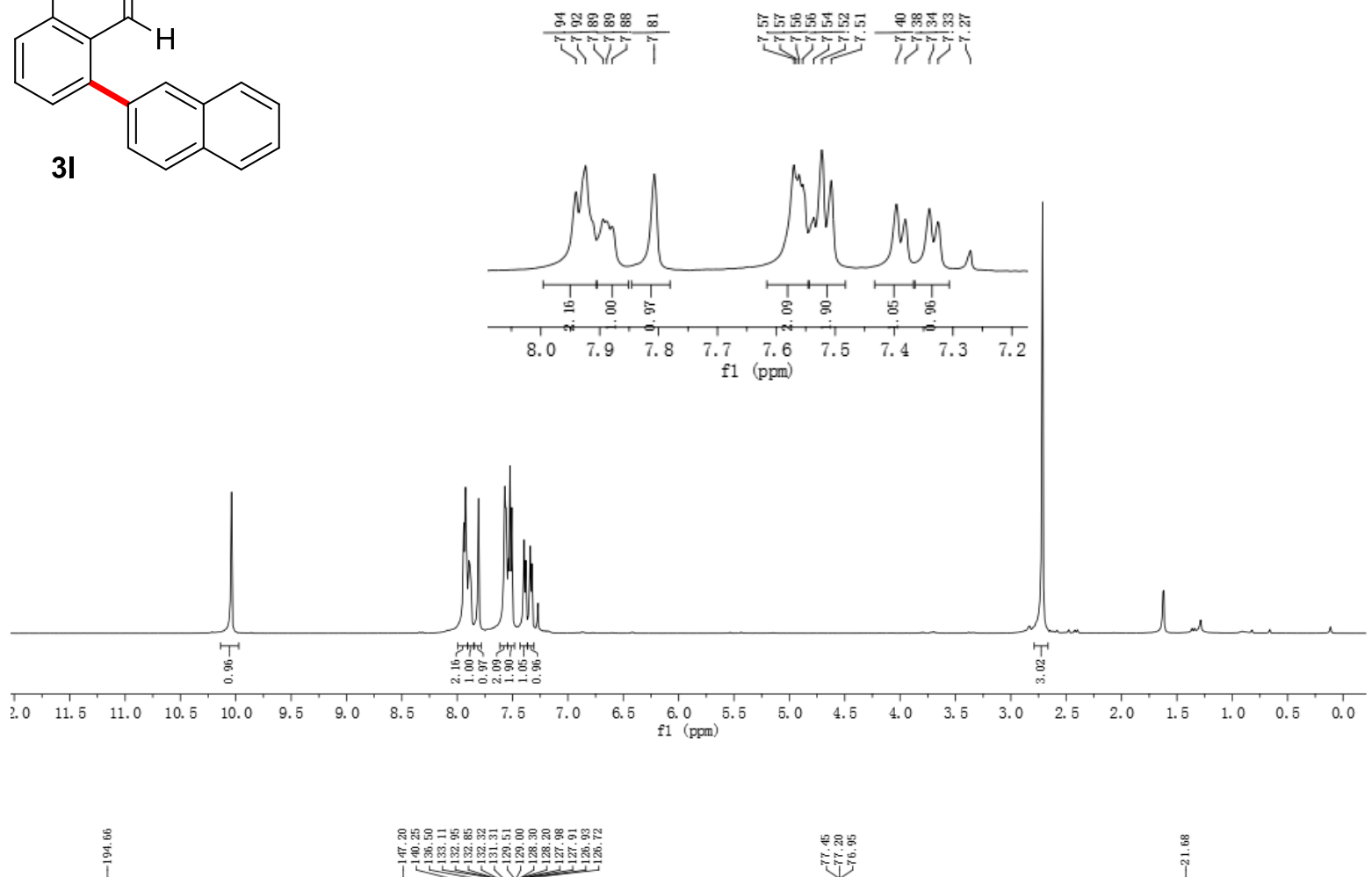

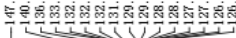

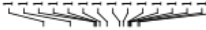<smiles>Cc1cccc(-c2ccc3ccccc3c2)c1C=O</smiles>
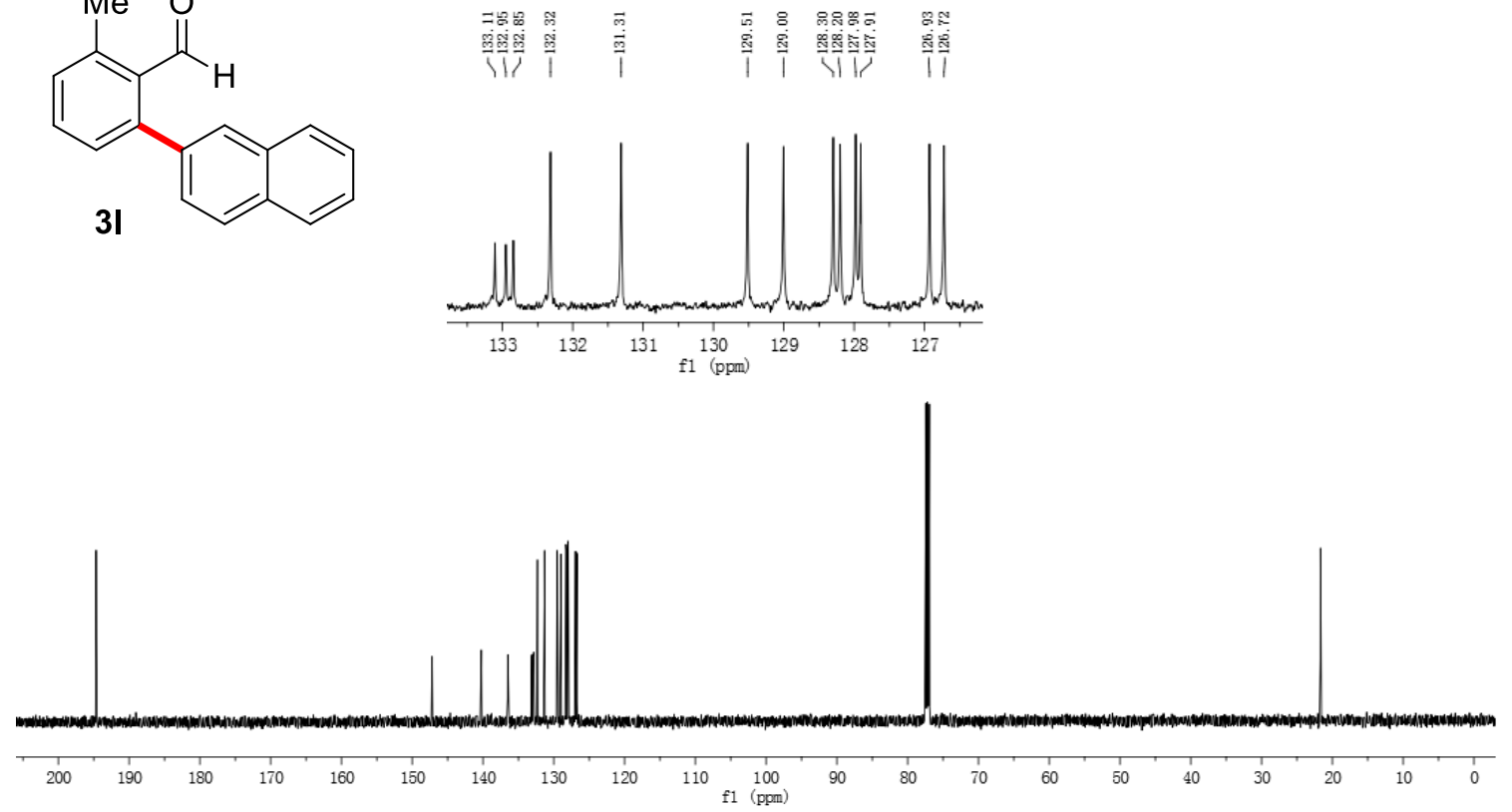

SI-49 


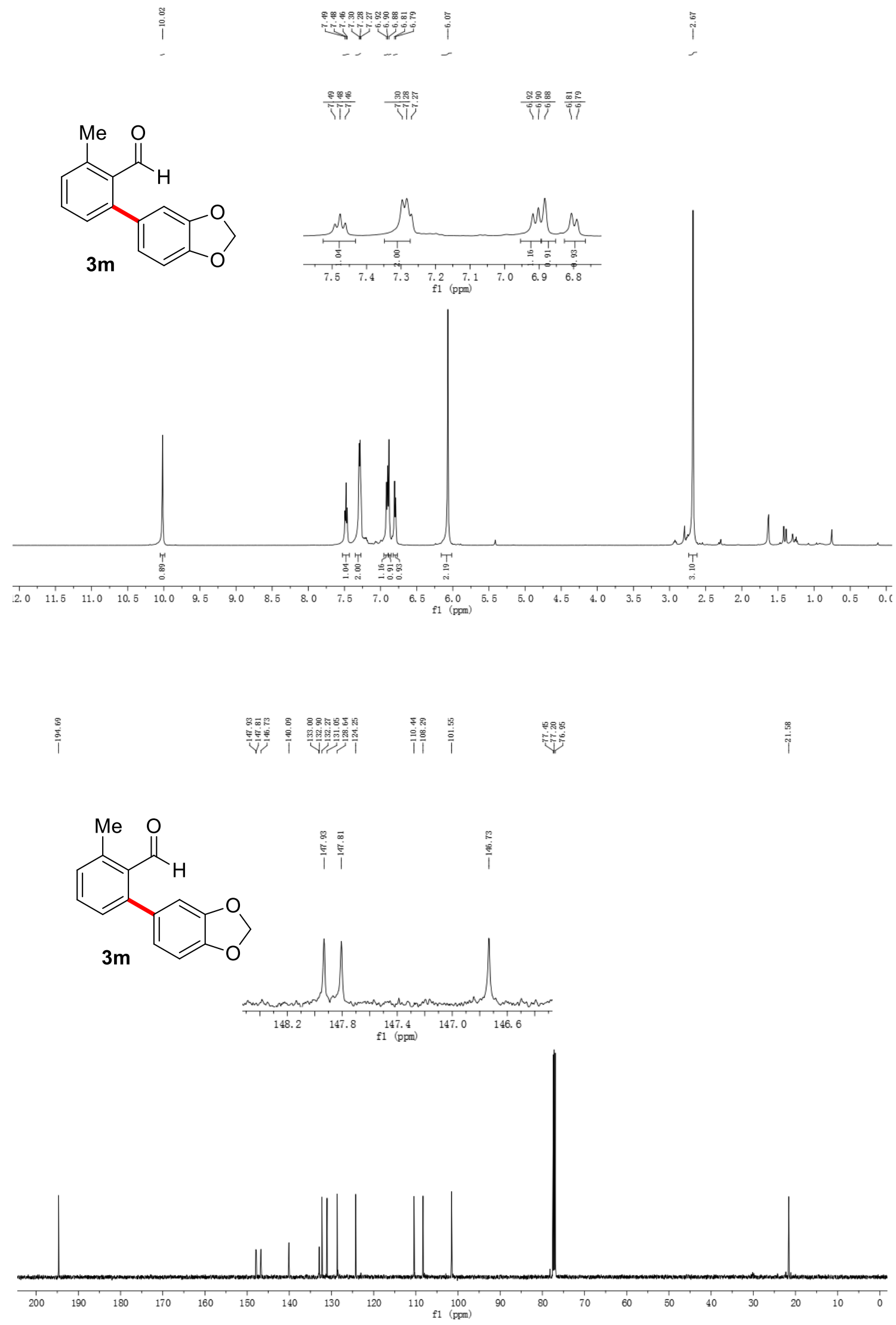

SI-50 
<smiles>Cc1cccc(-c2ccoc2)c1C=O</smiles>
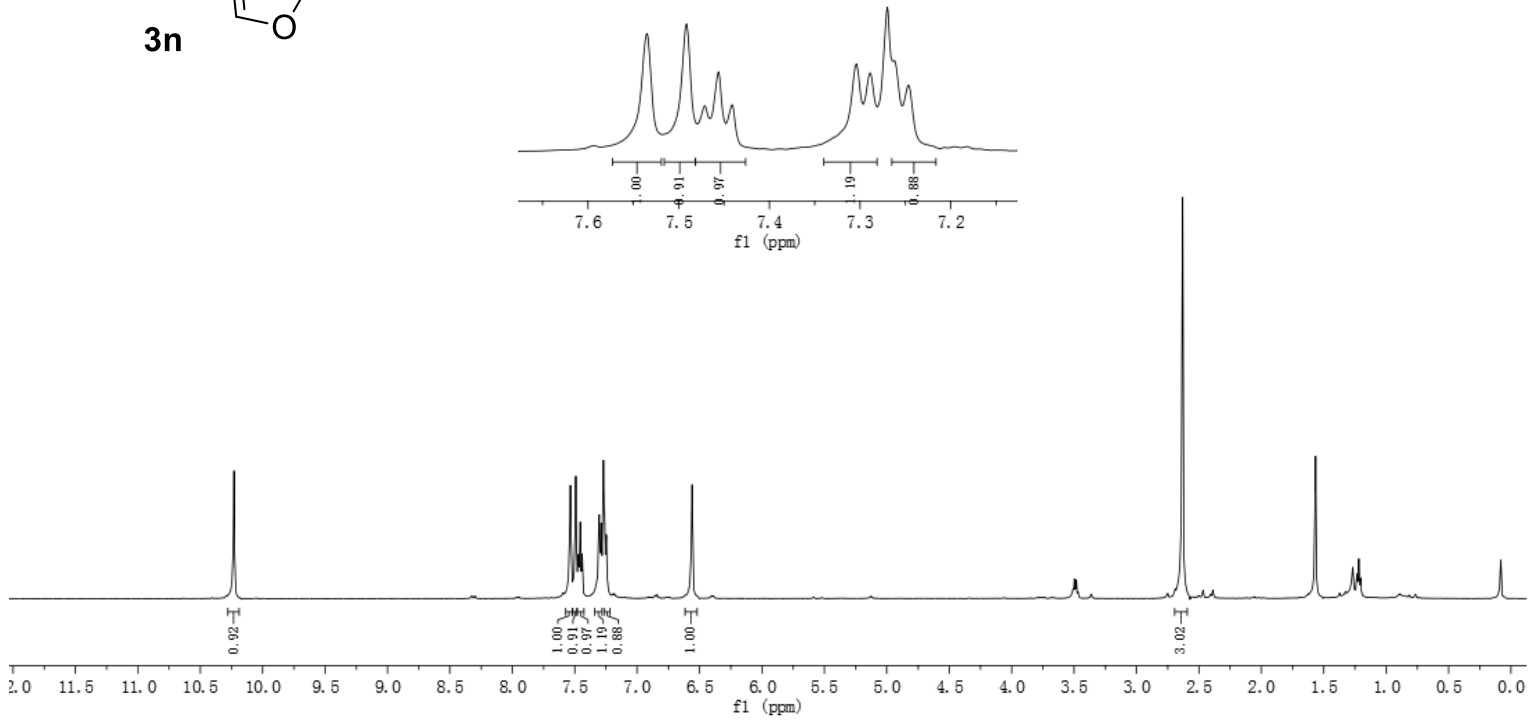<smiles>Cc1cccc(-c2ccoc2)c1C=O</smiles>

$3 n$

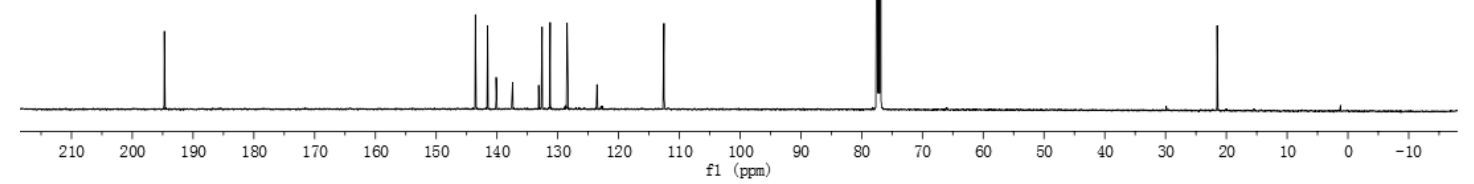




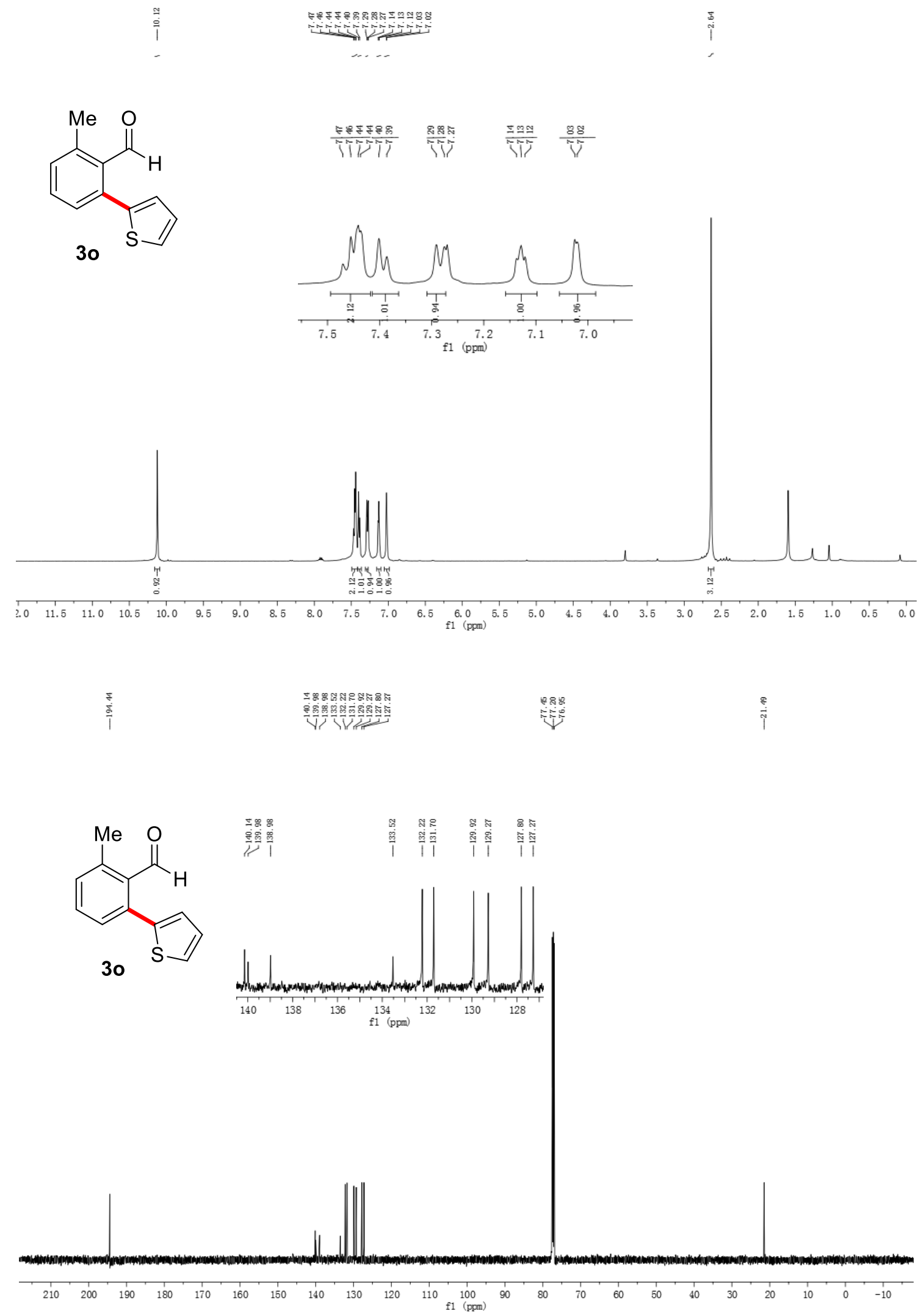




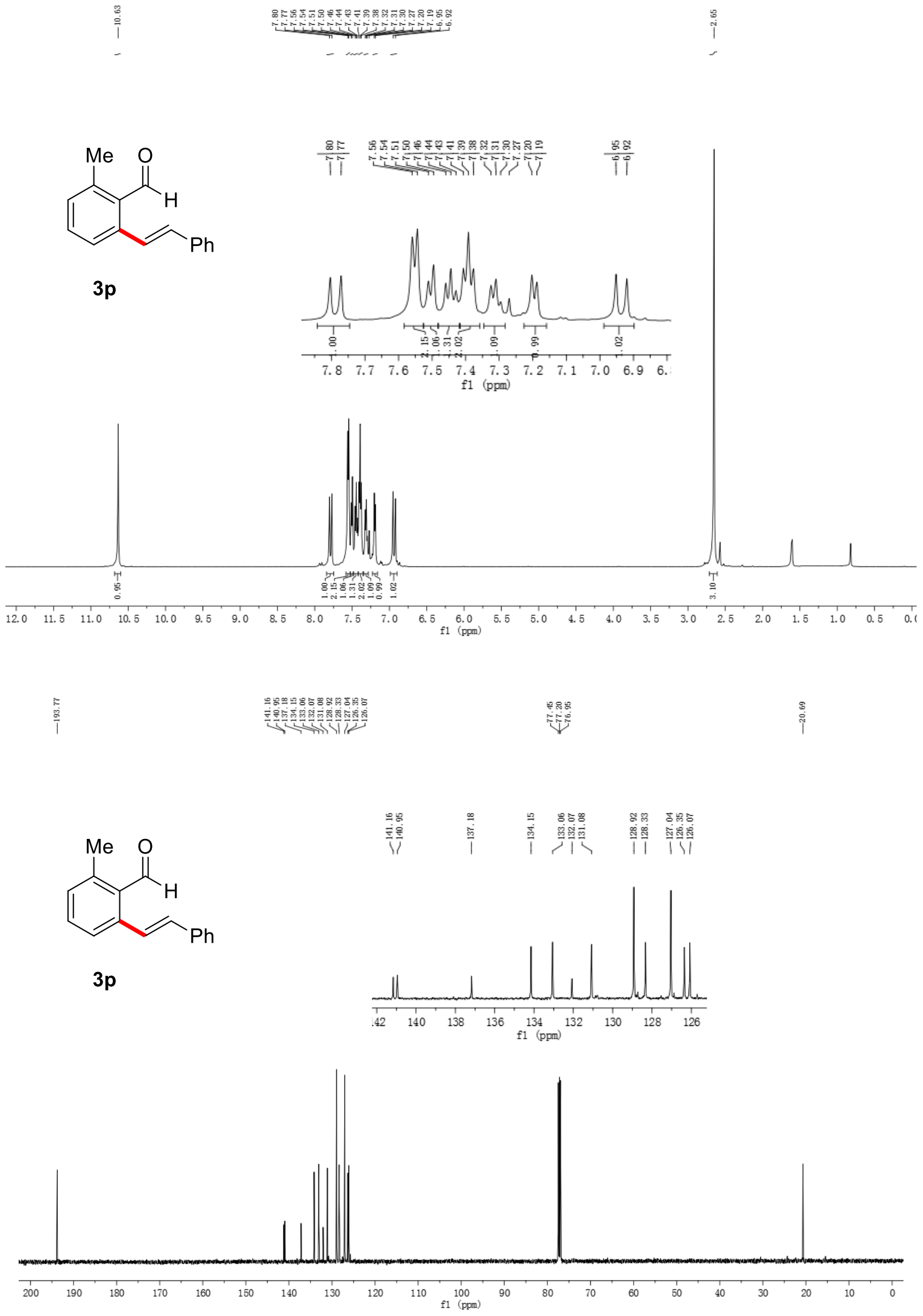

SI-53 


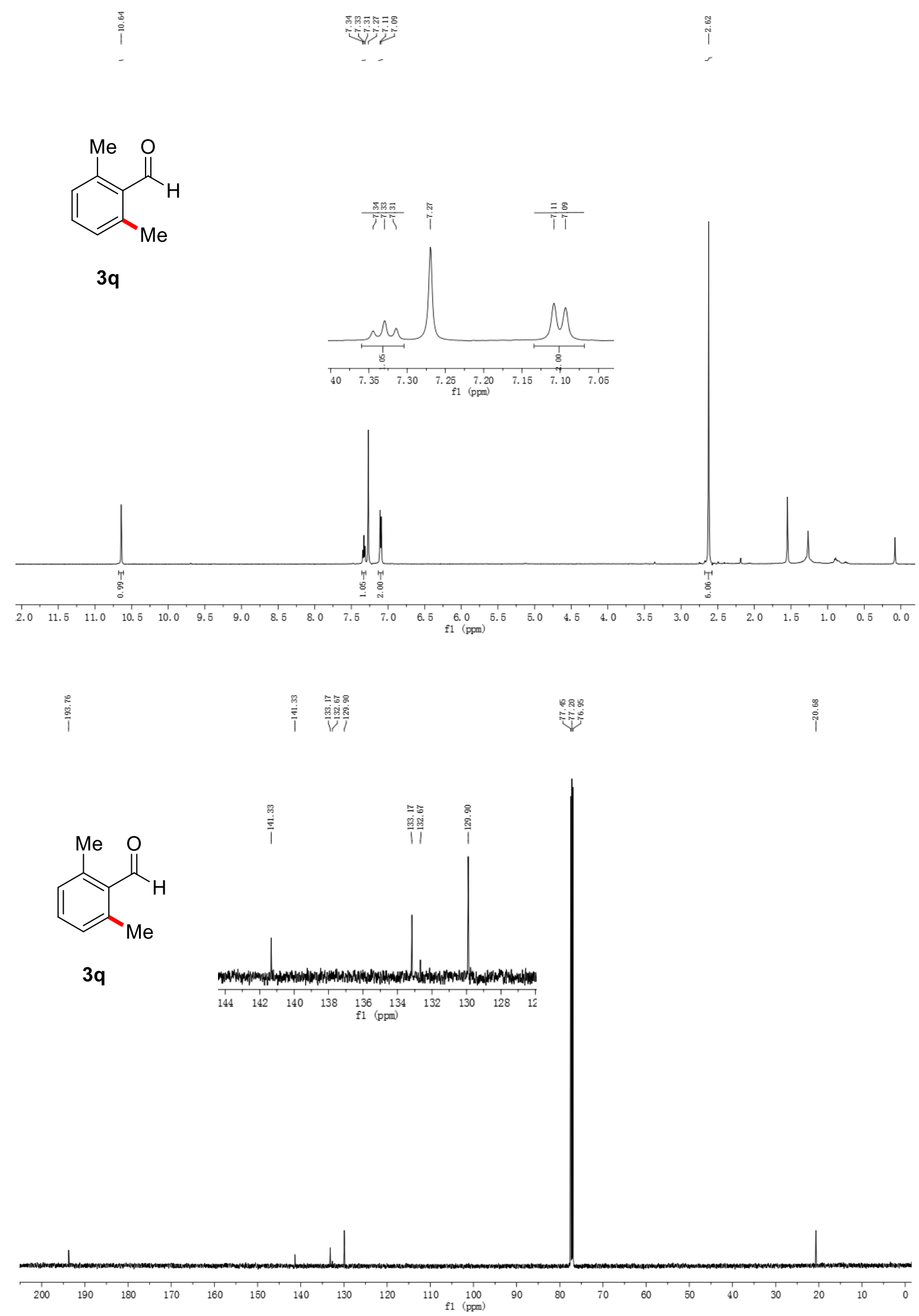

SI-54 


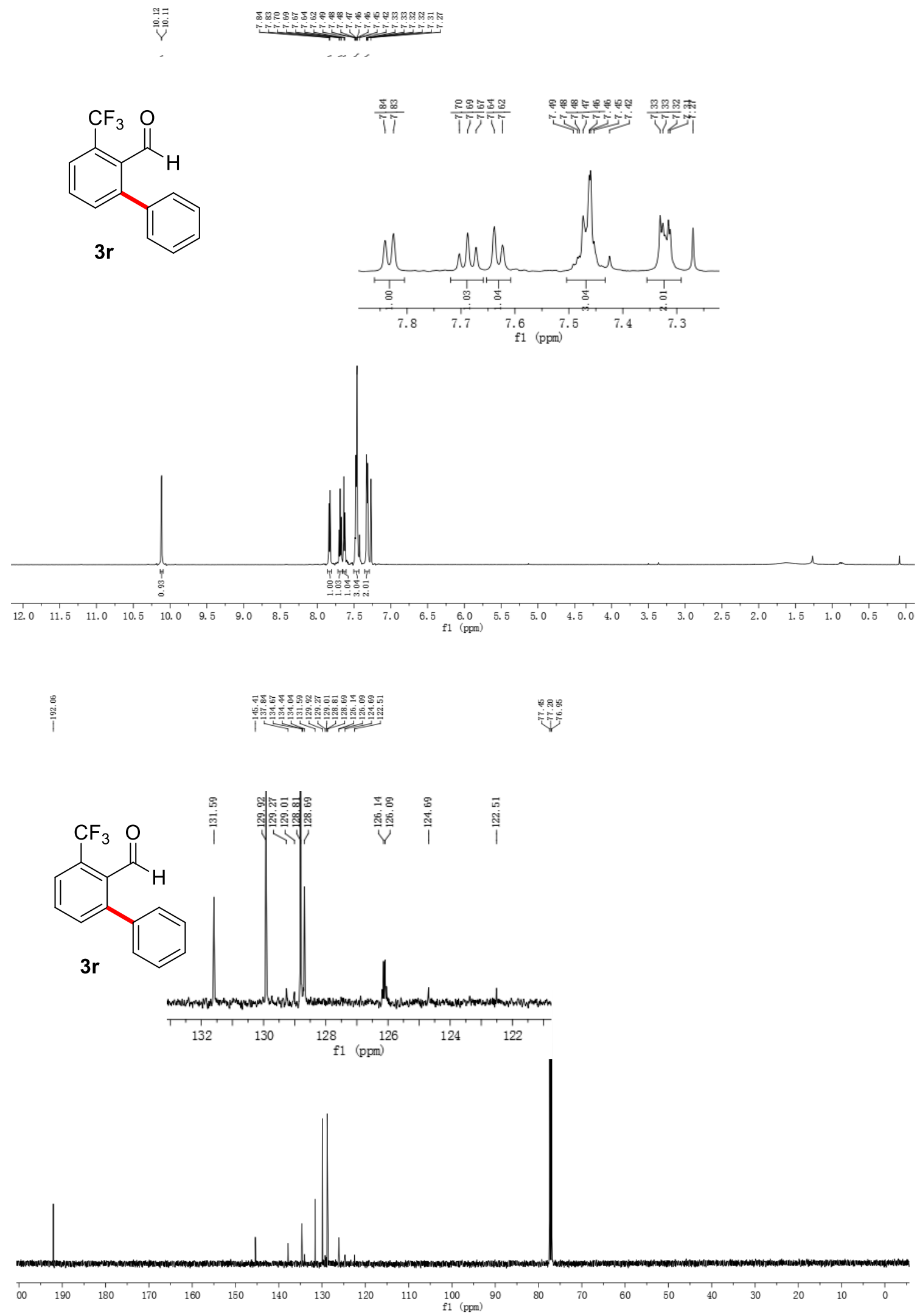

SI-55 
<smiles>O=Cc1c(-c2ccccc2)cccc1C(F)(F)F</smiles>

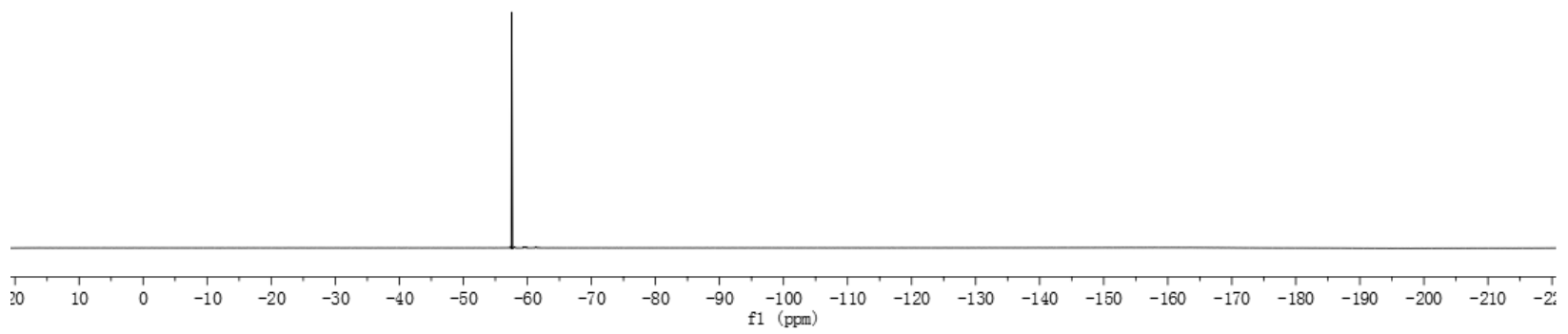




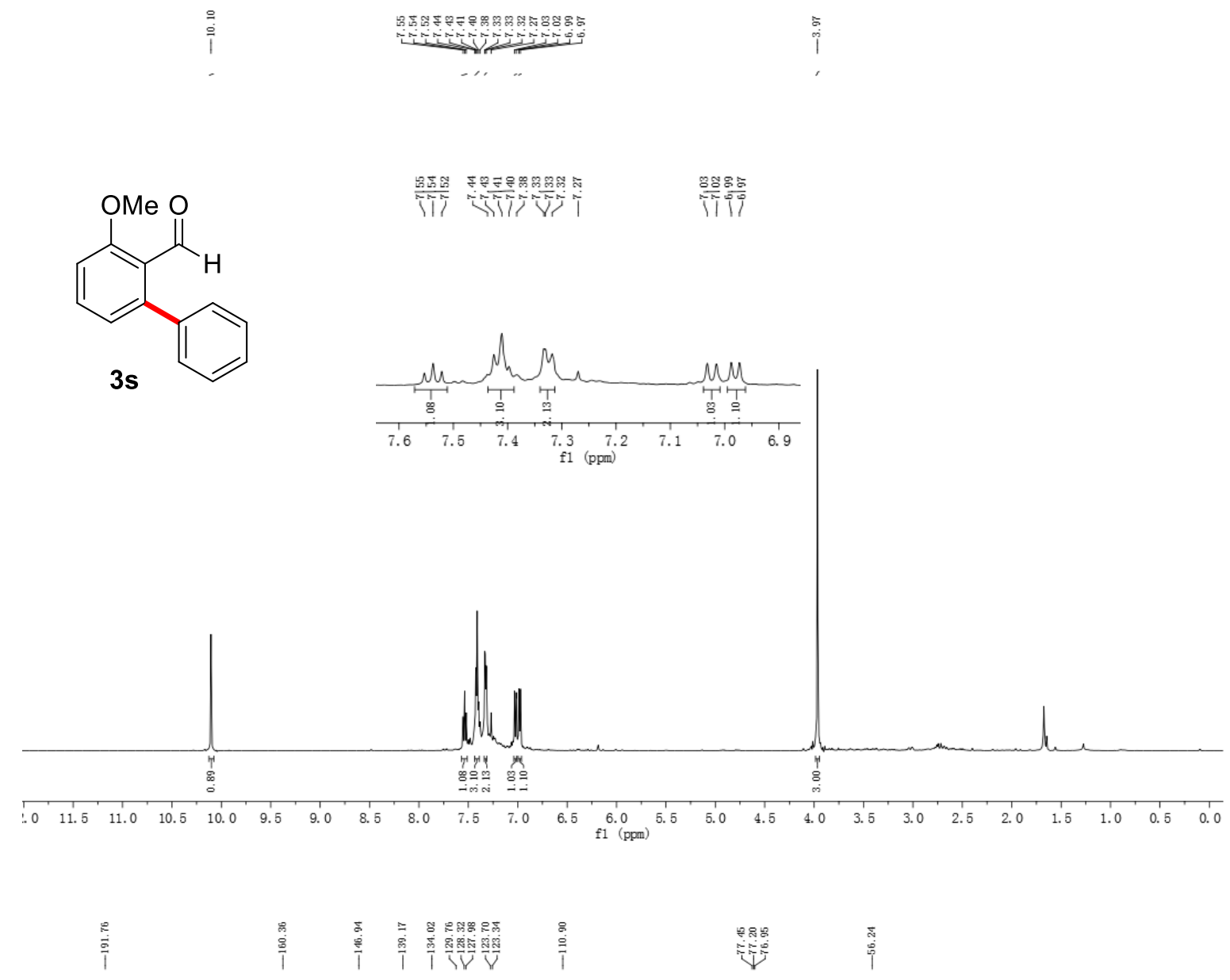<smiles>COc1cccc(-c2ccccc2)c1C=O</smiles>

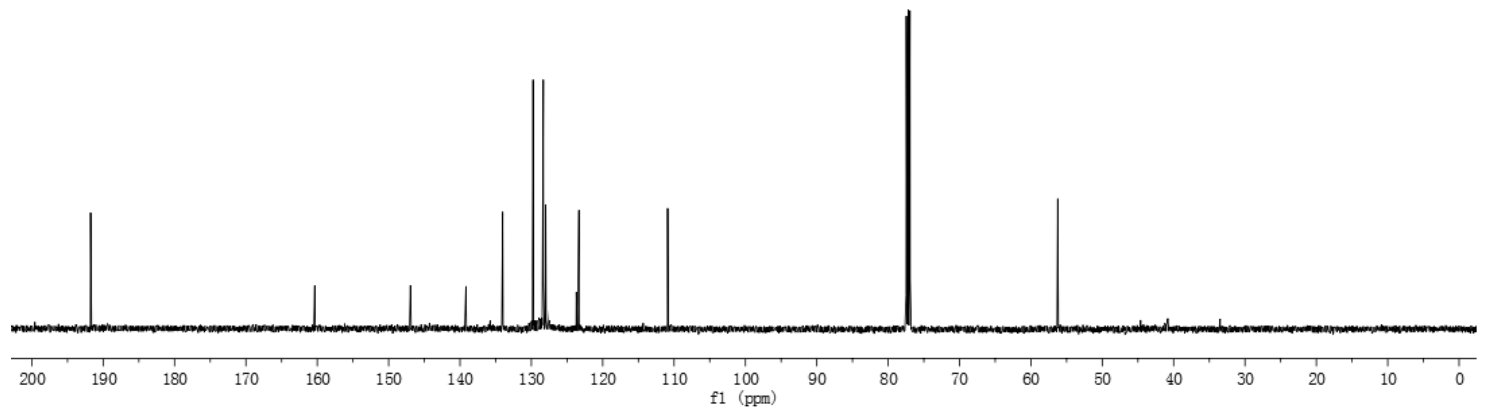




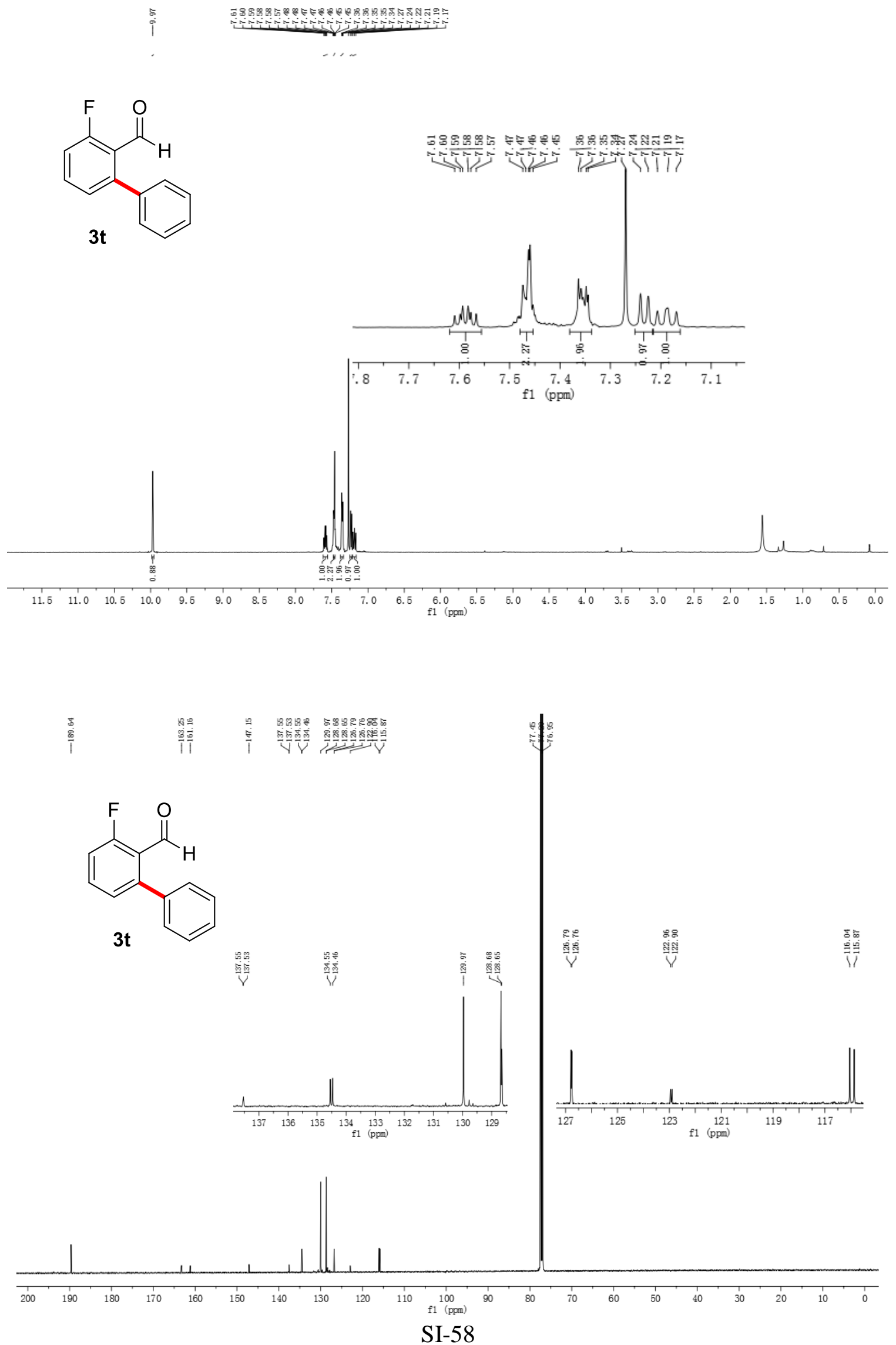




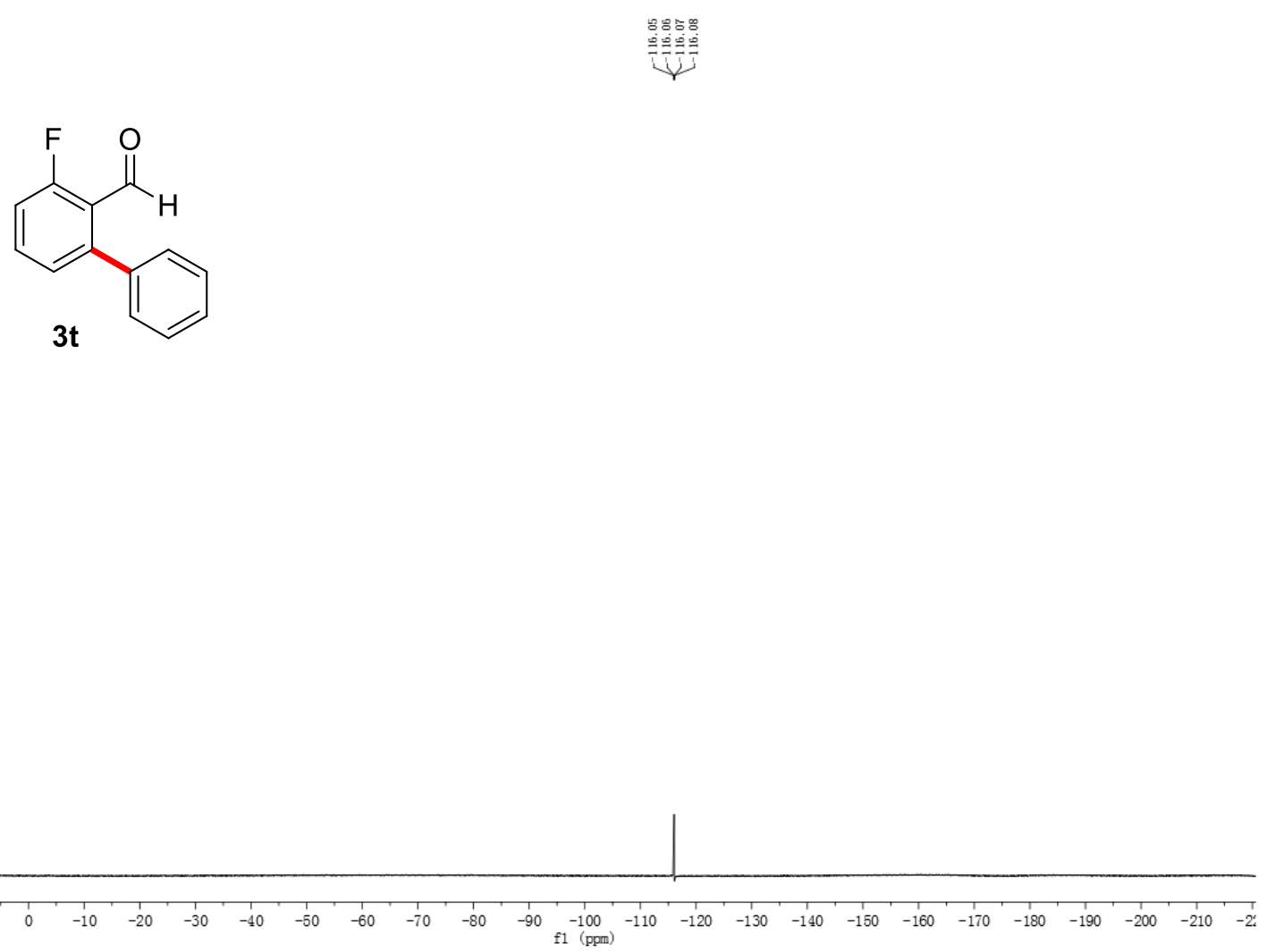




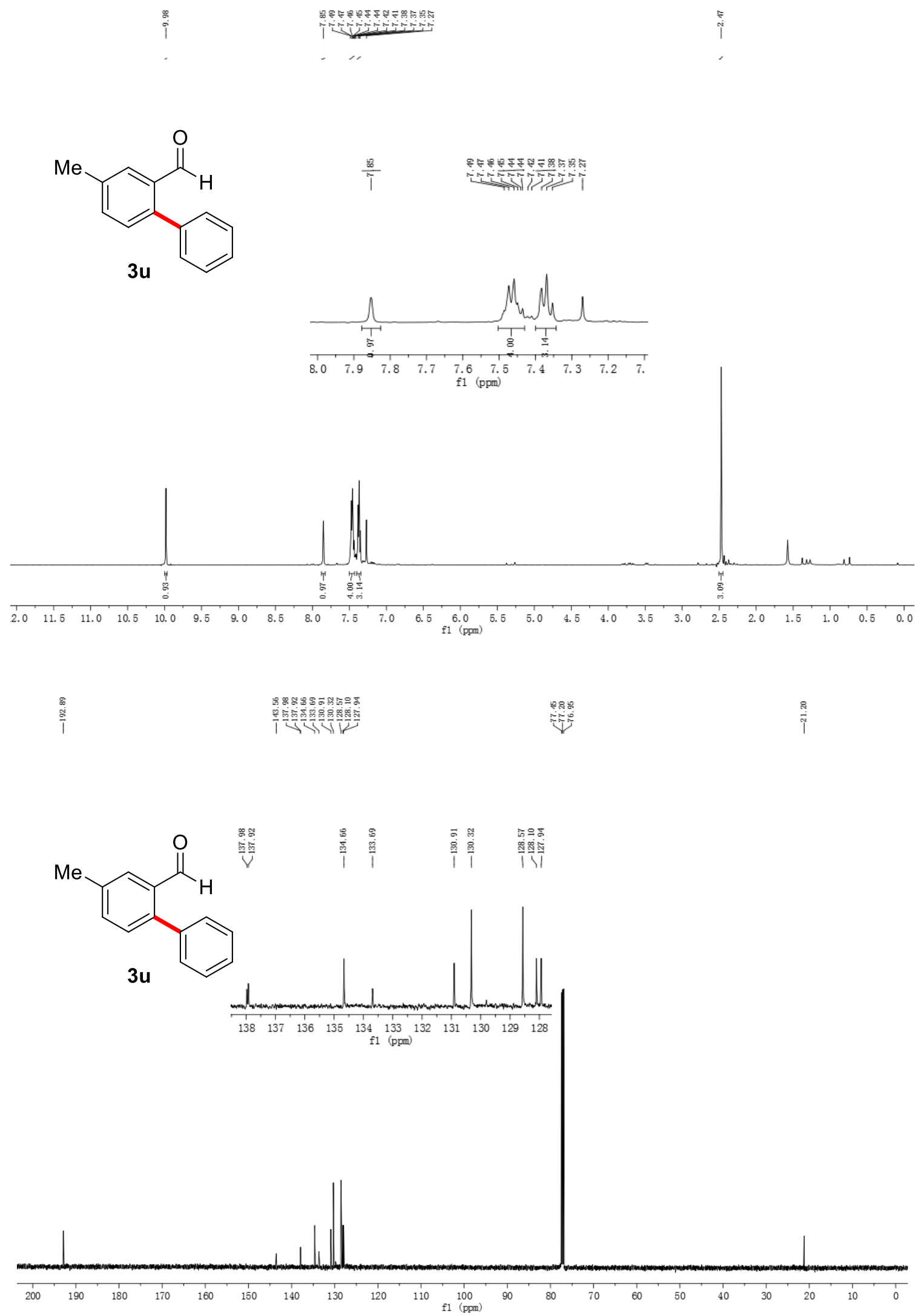




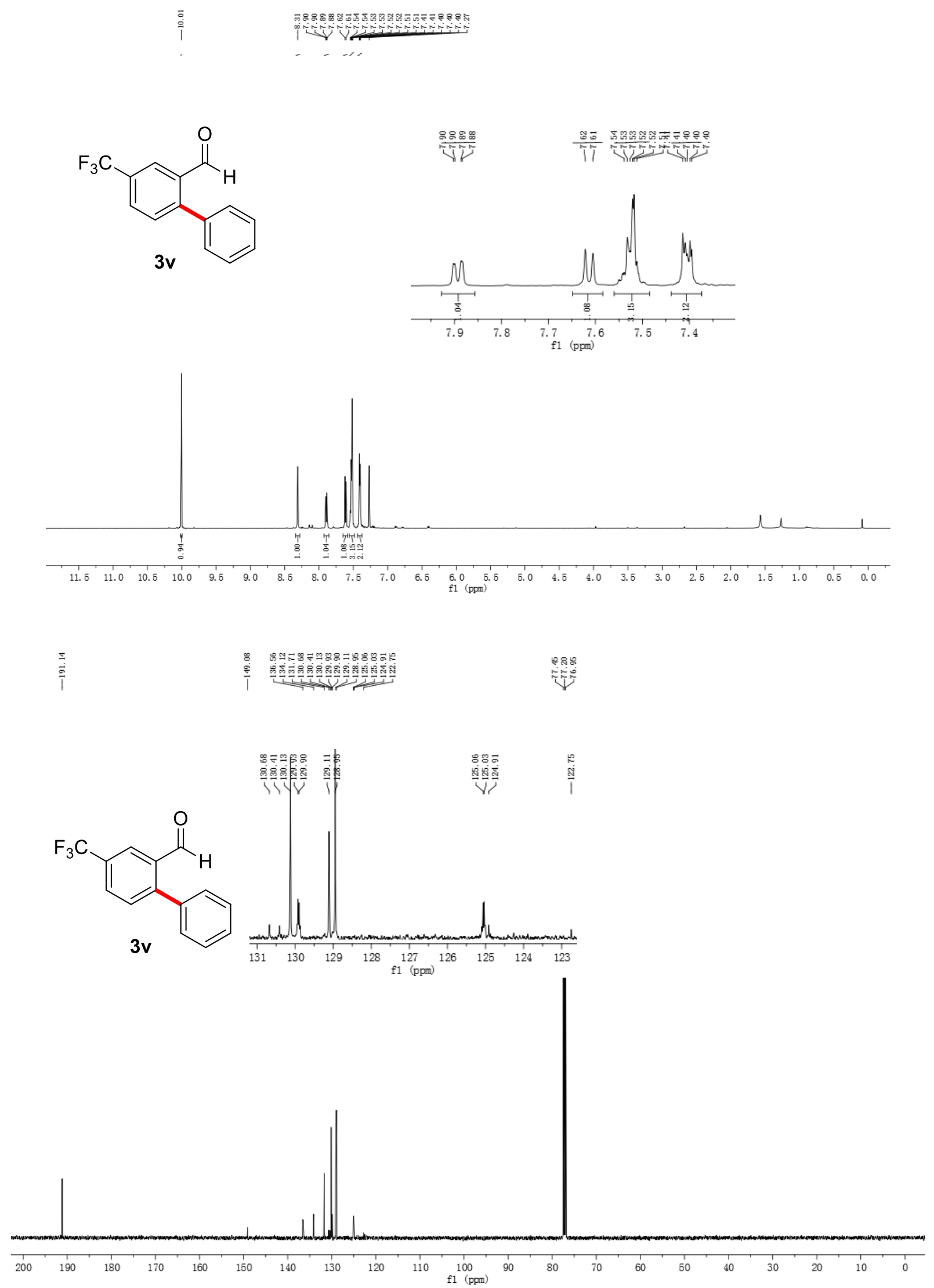

SI-61 
<smiles>O=Cc1cc(C(F)(F)F)ccc1-c1ccccc1</smiles>

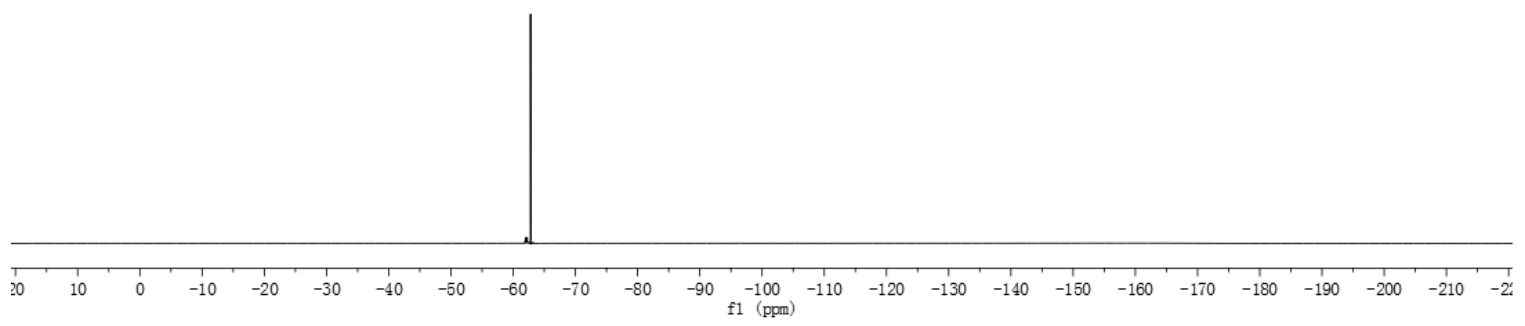


<smiles>COc1ccc(-c2ccccc2)c(C=O)c1-c1ccccc1</smiles>

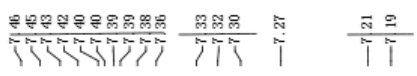

$3 w$
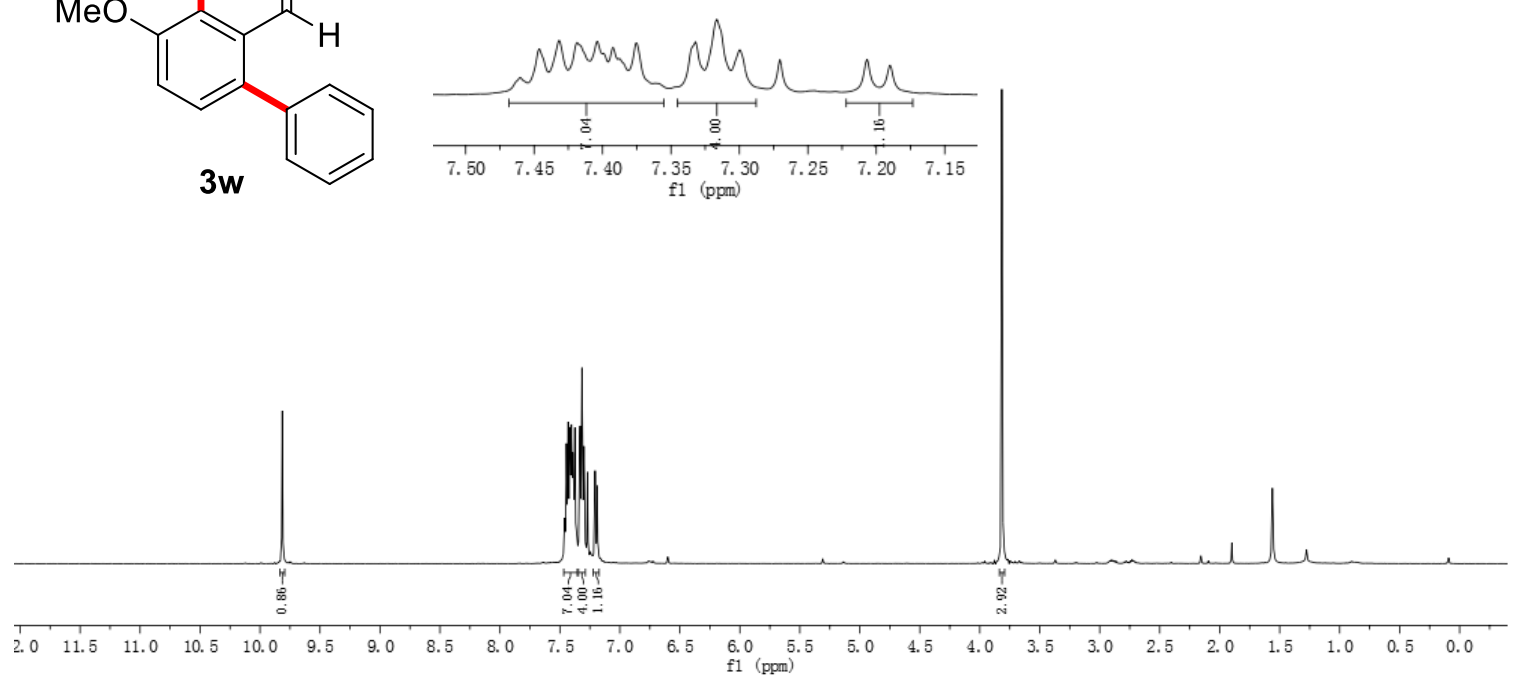<smiles>COc1ccc(-c2ccccc2)c(C=O)c1-c1ccccc1</smiles>

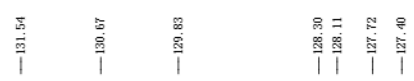

$3 w$
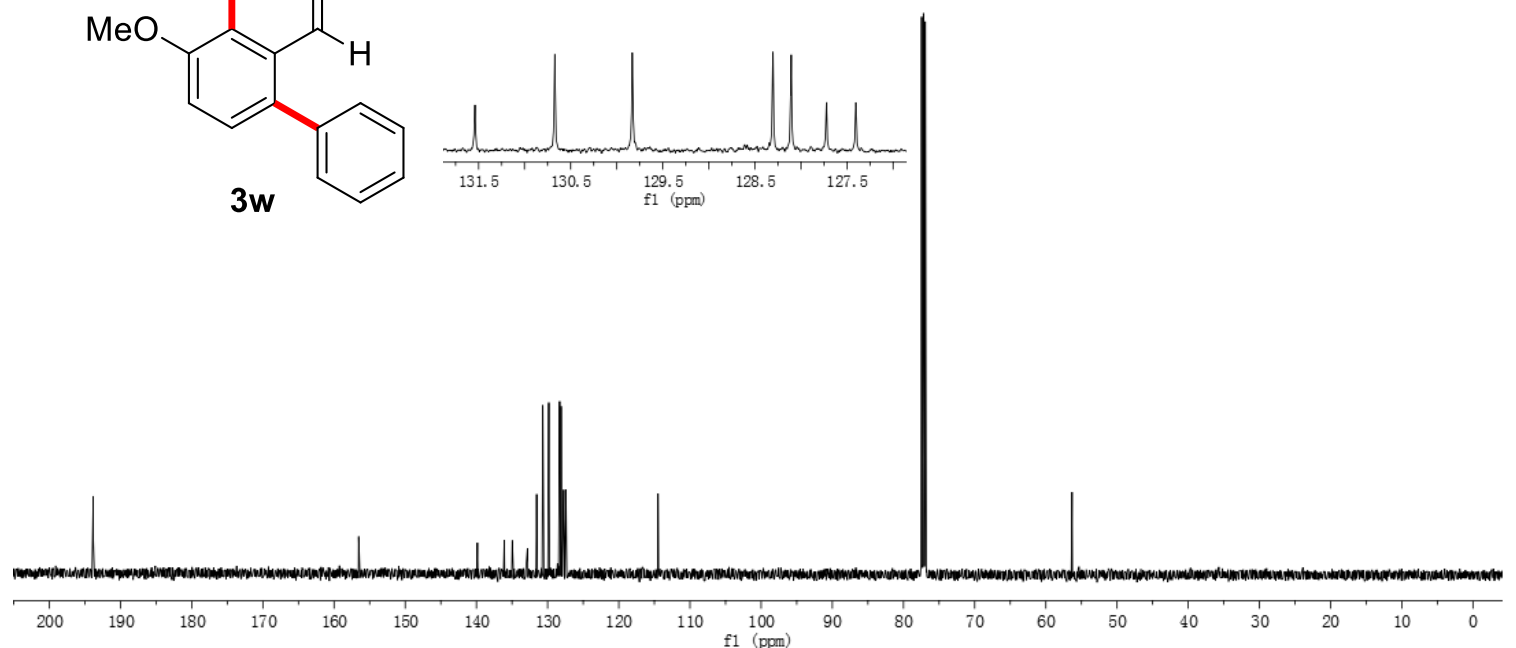


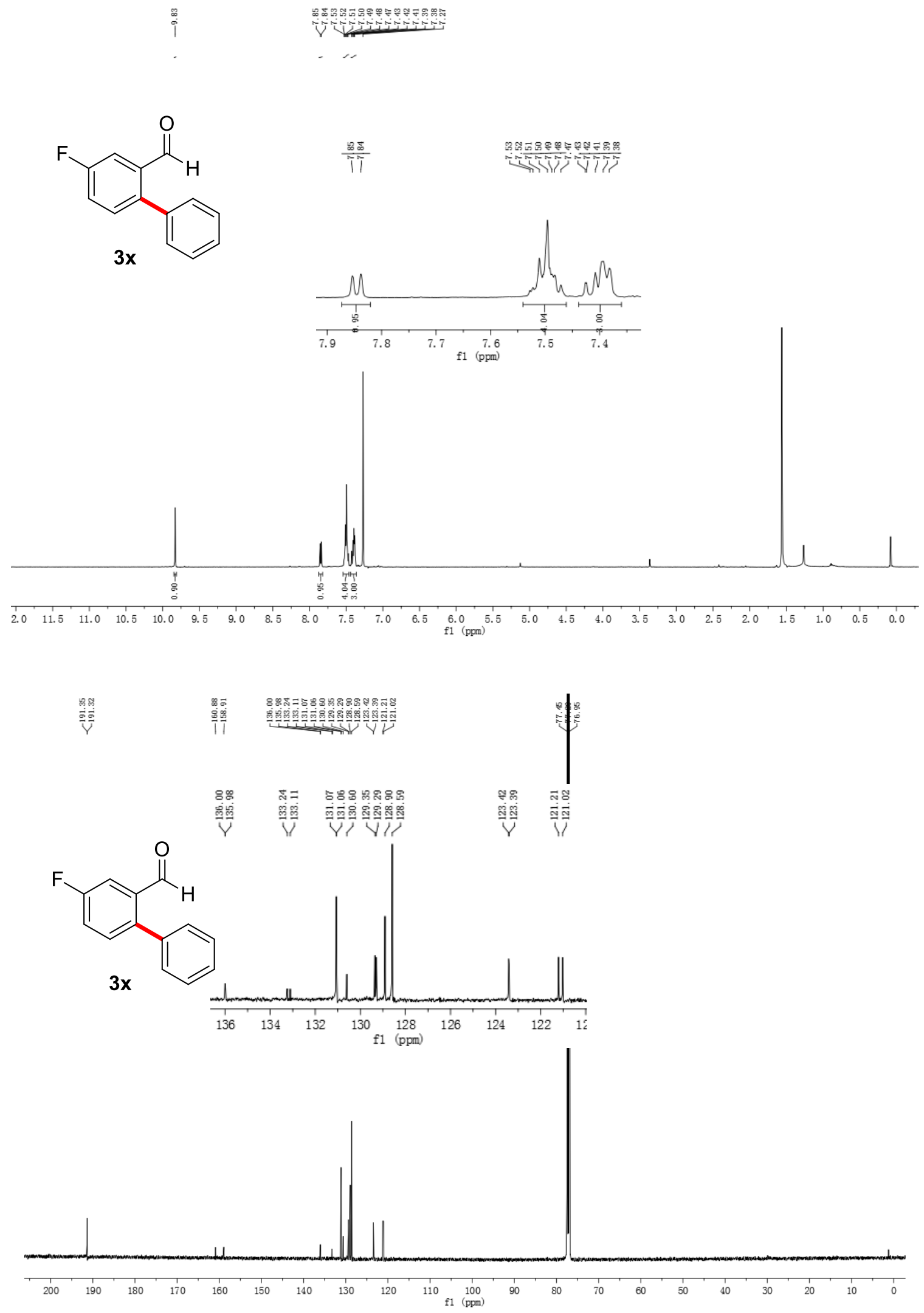



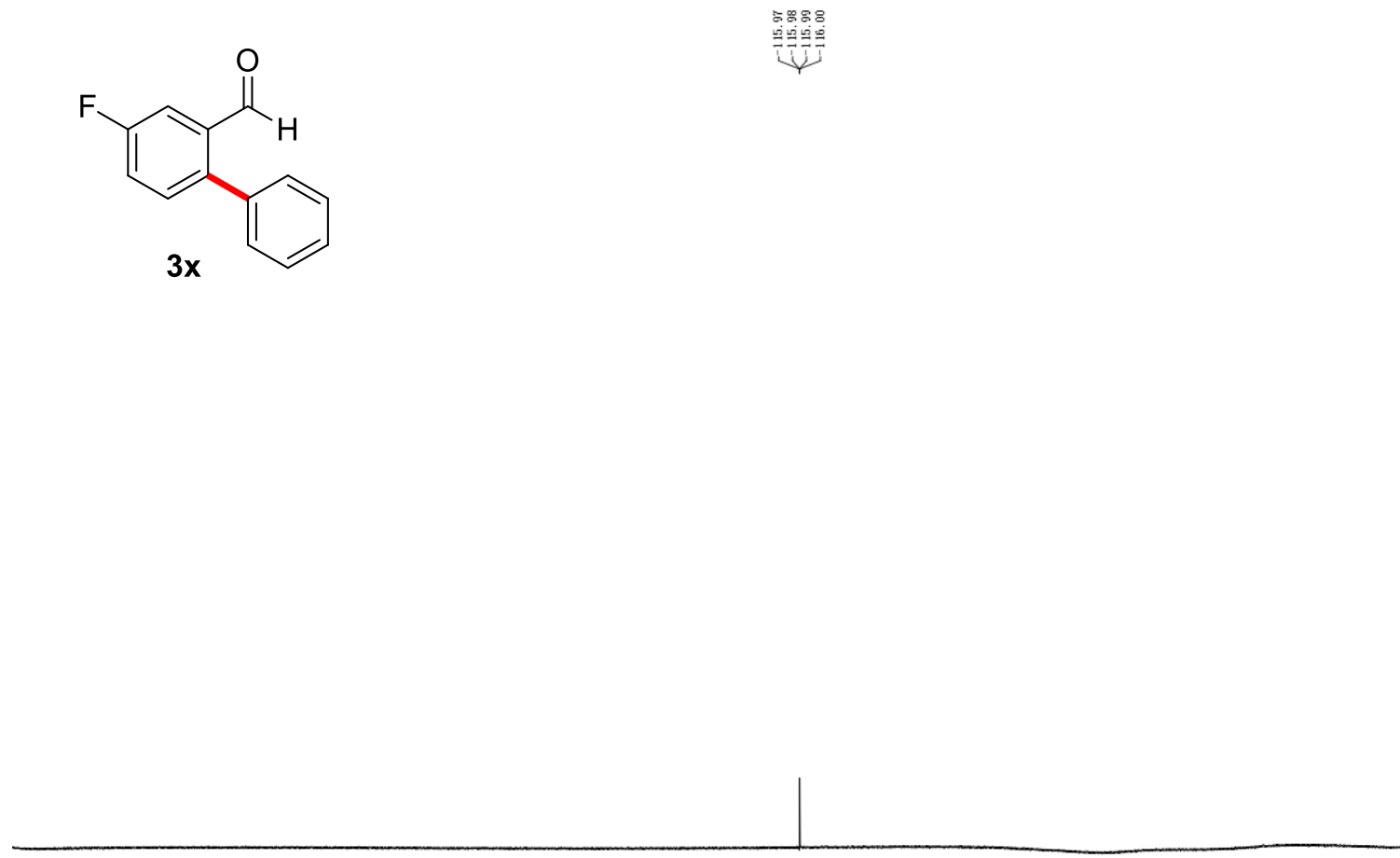

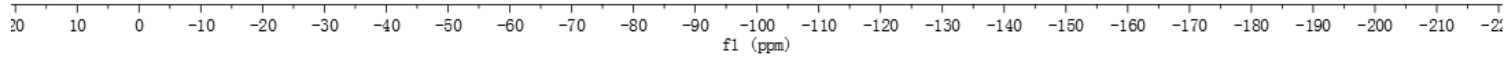




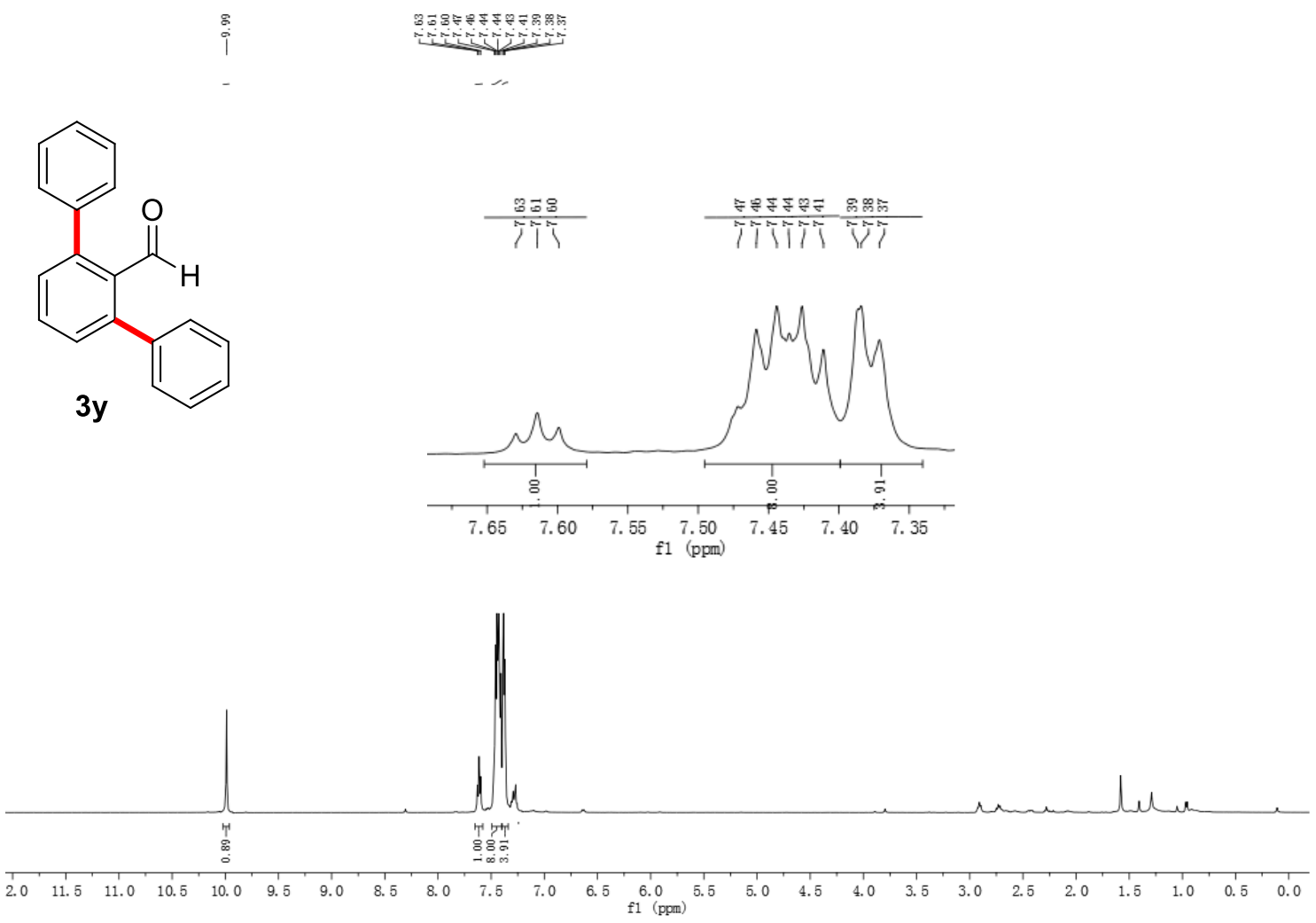

$\stackrel{5}{i}$

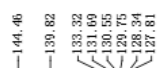

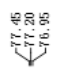

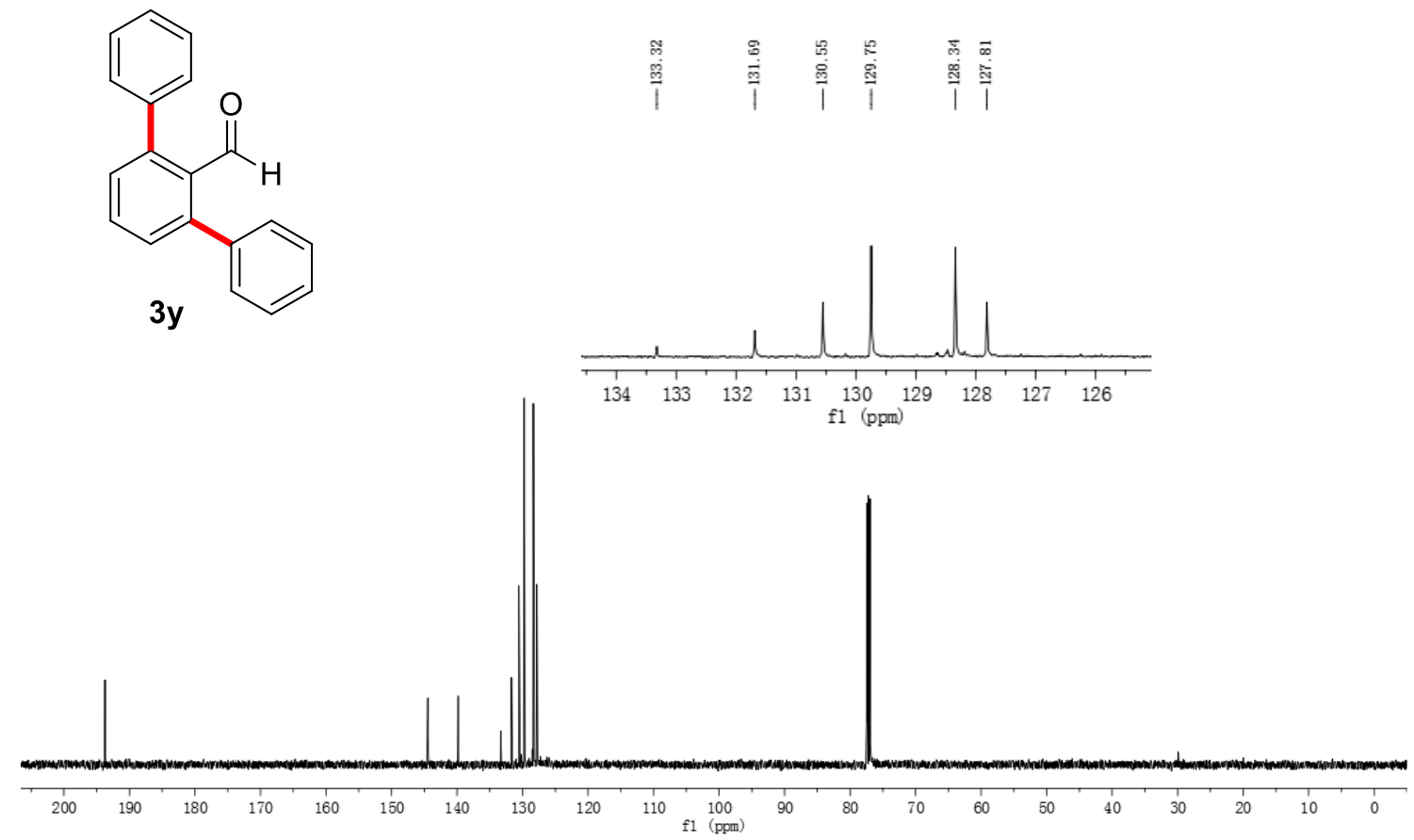




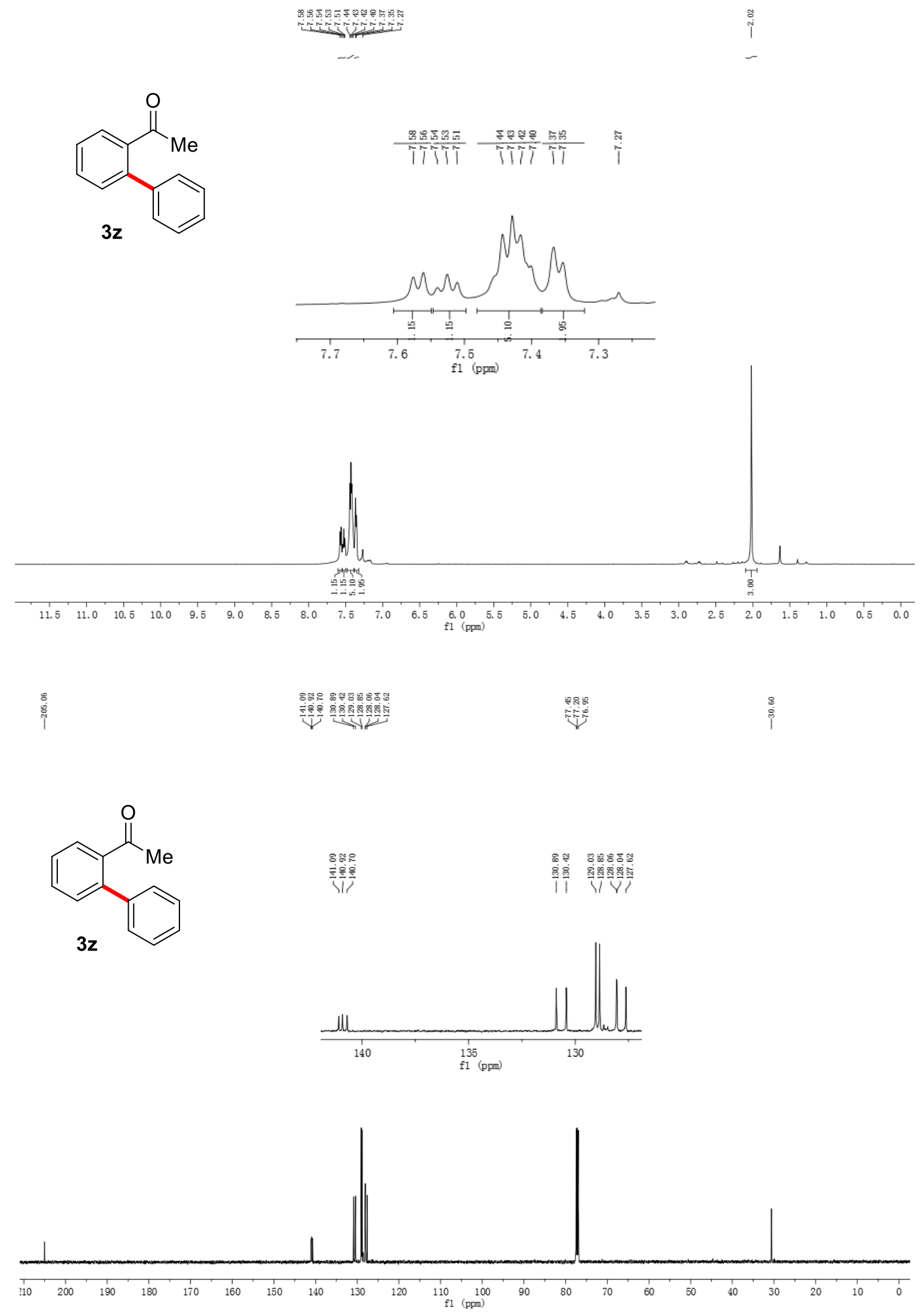

SI-67 


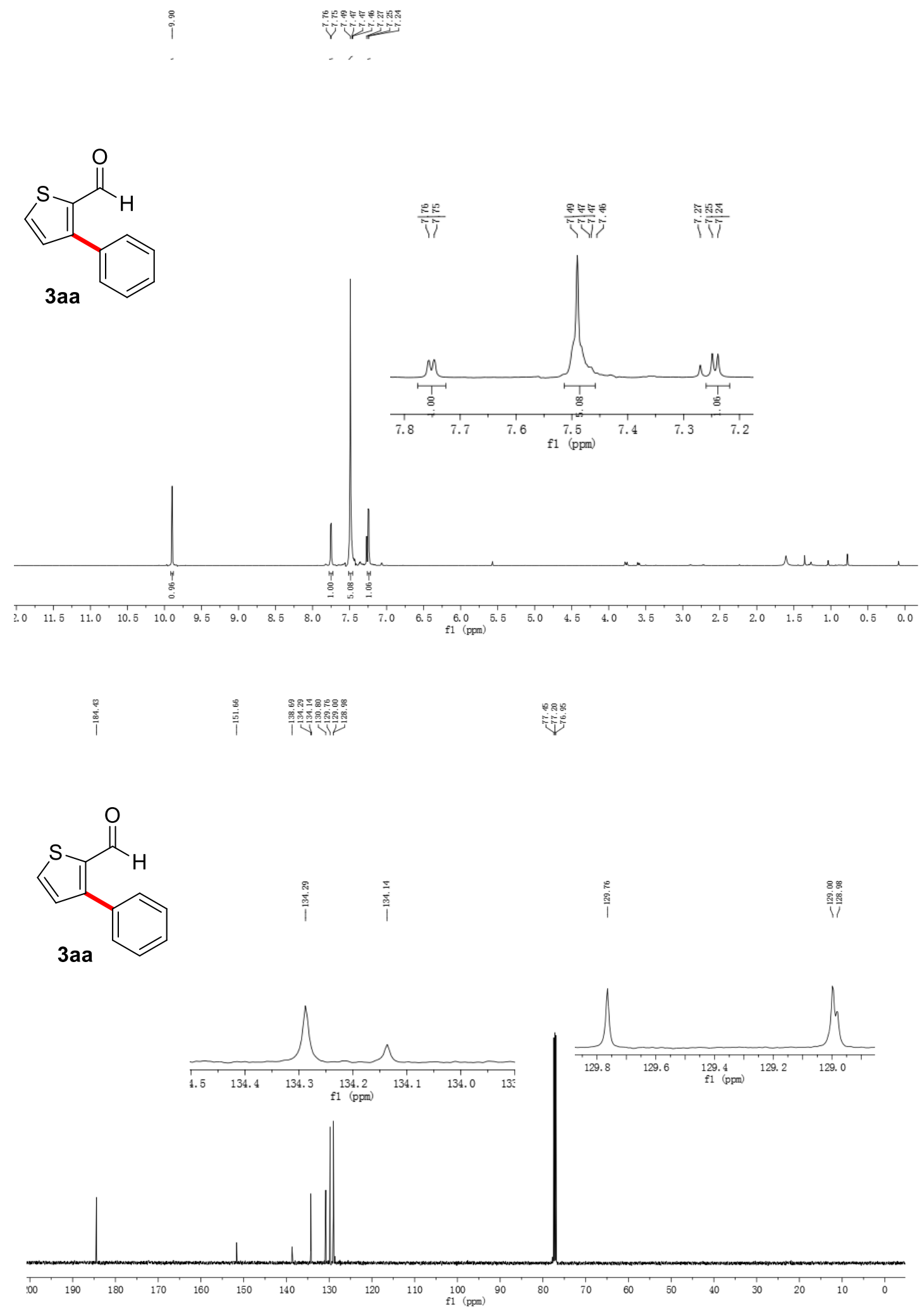




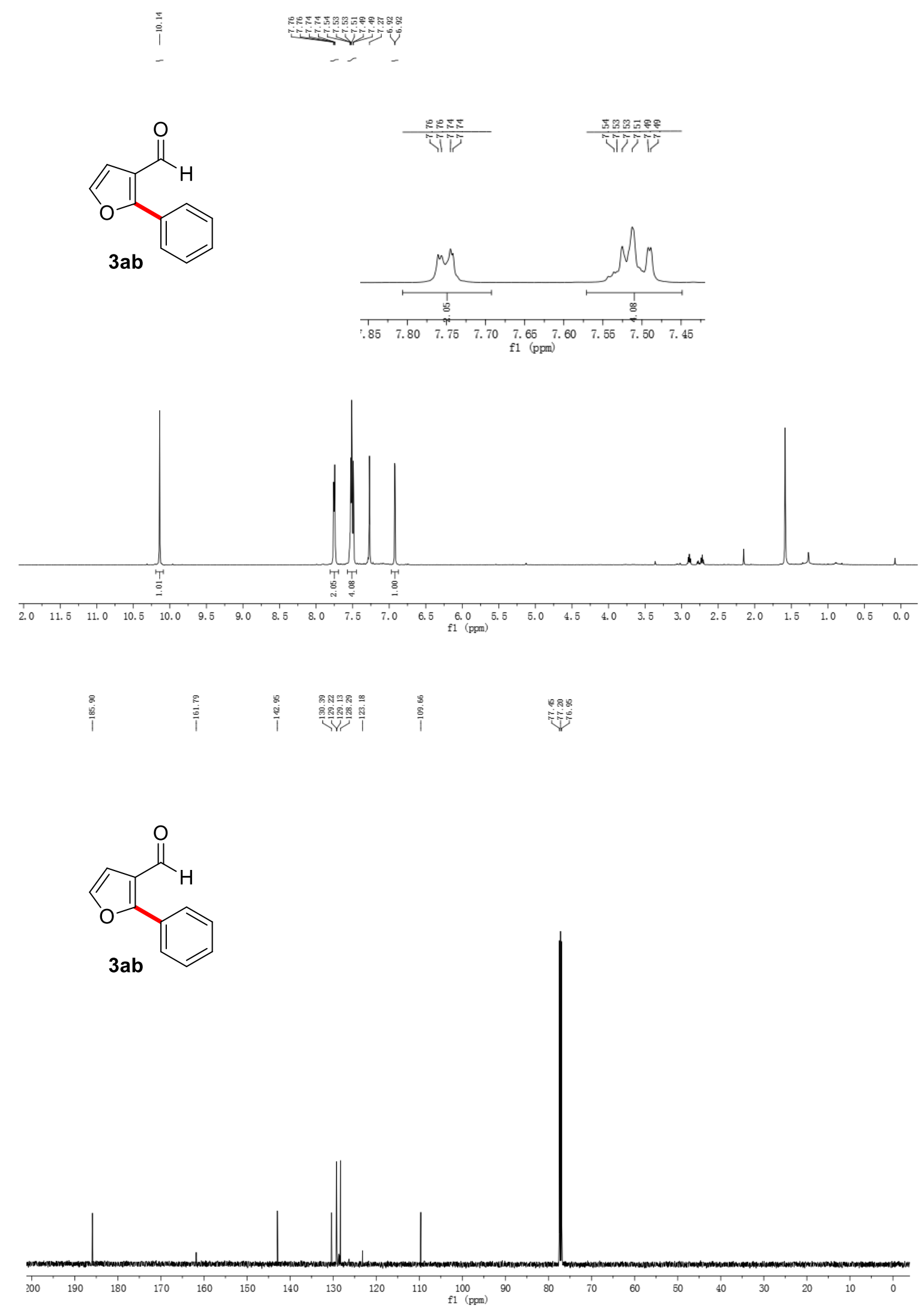


1<smiles>O=Cc1ccc2ccccc2c1-c1ccccc1</smiles>

3ac
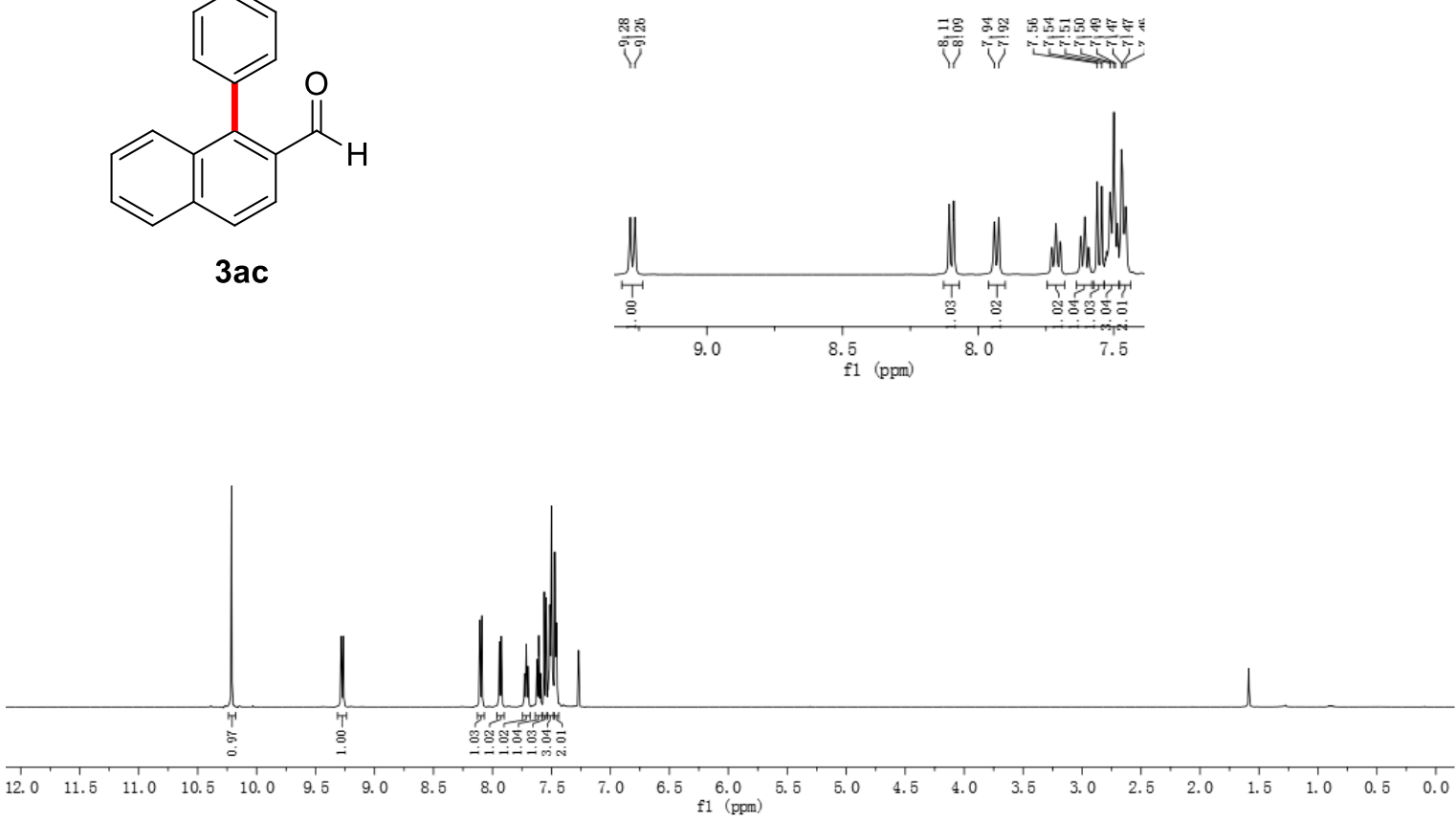

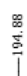

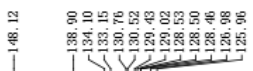

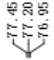<smiles>O=Cc1ccc2ccccc2c1-c1ccccc1</smiles>

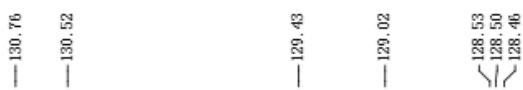

3ac
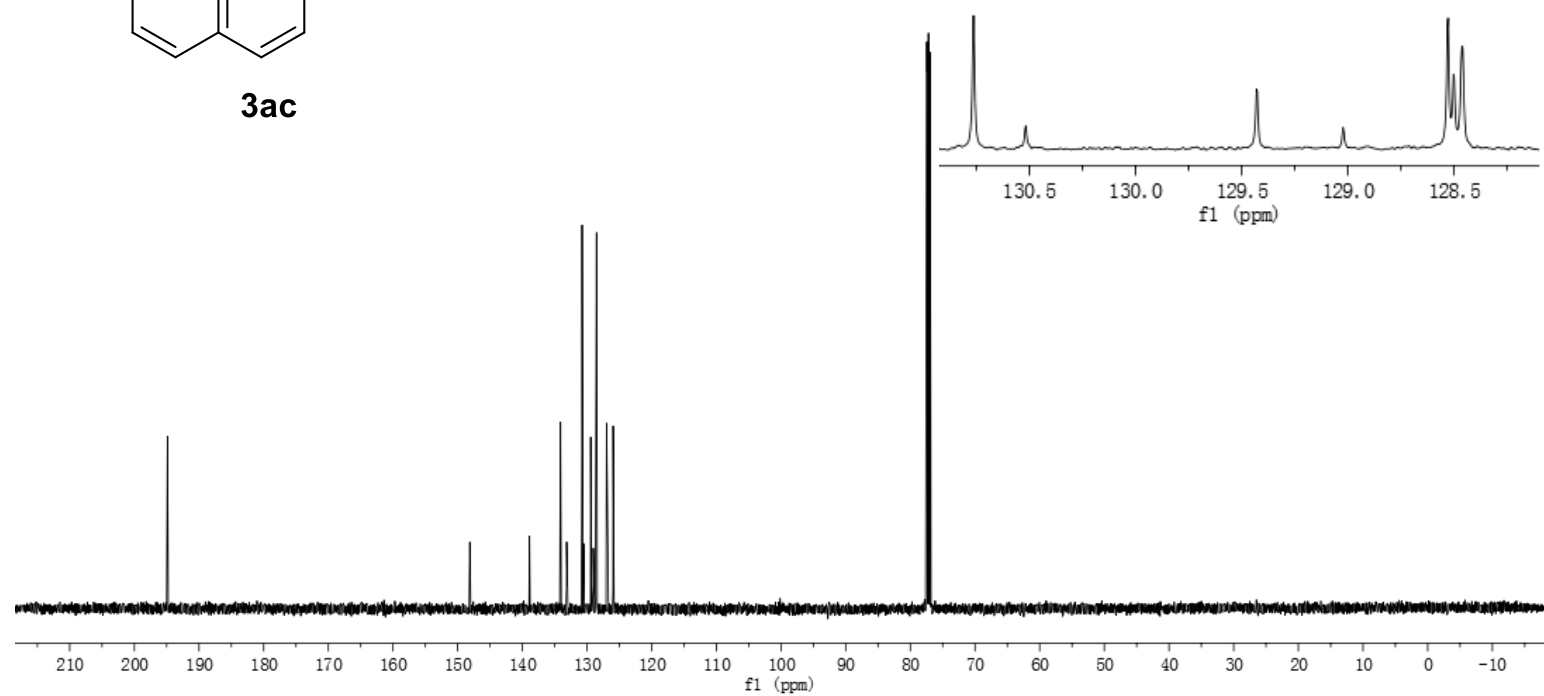

SI-70 


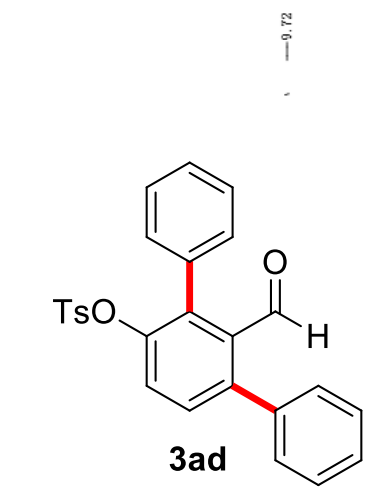

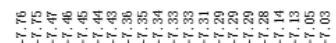

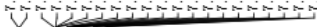

- , ....

i⿱ 


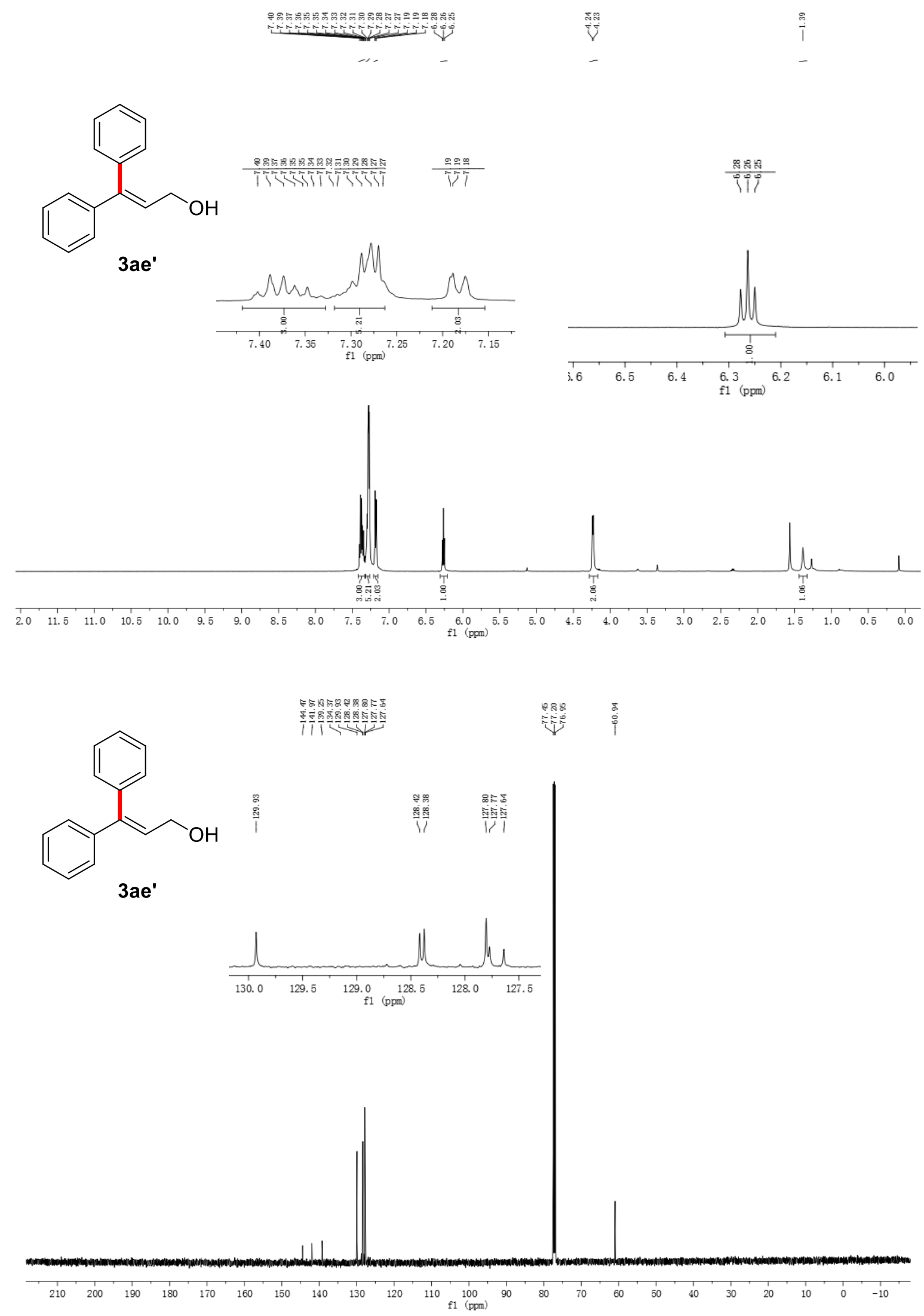

SI-72 


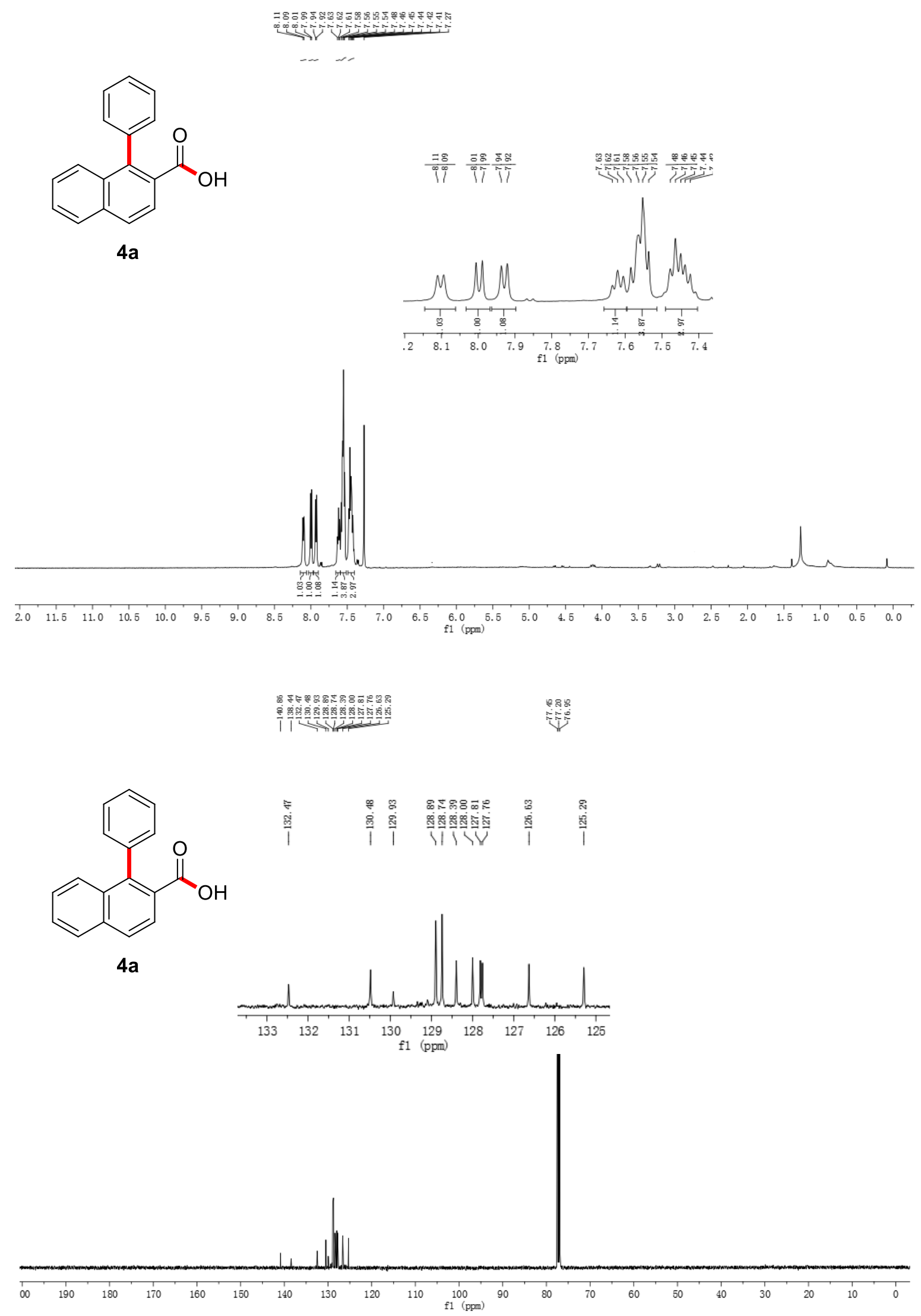

SI-73 


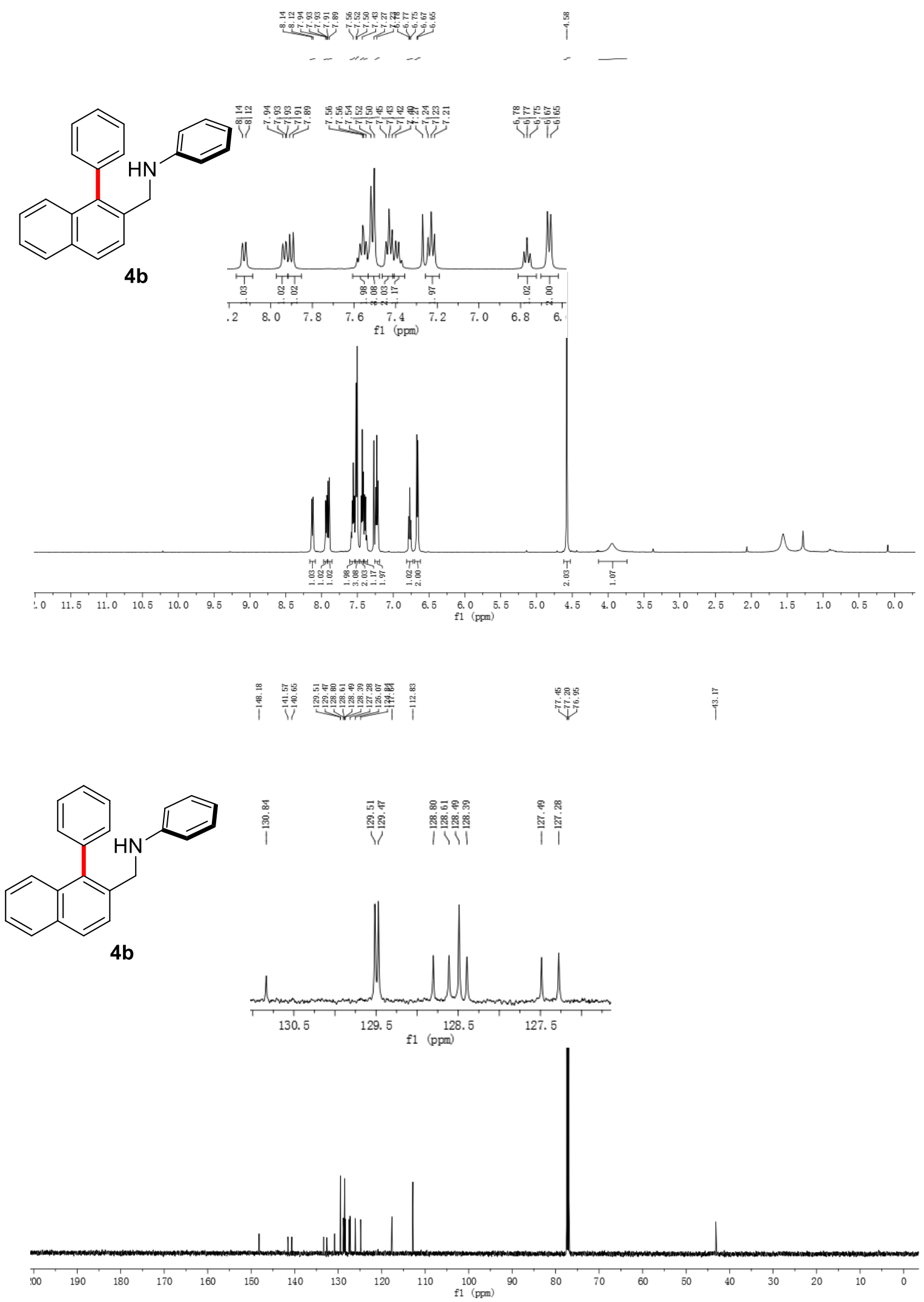




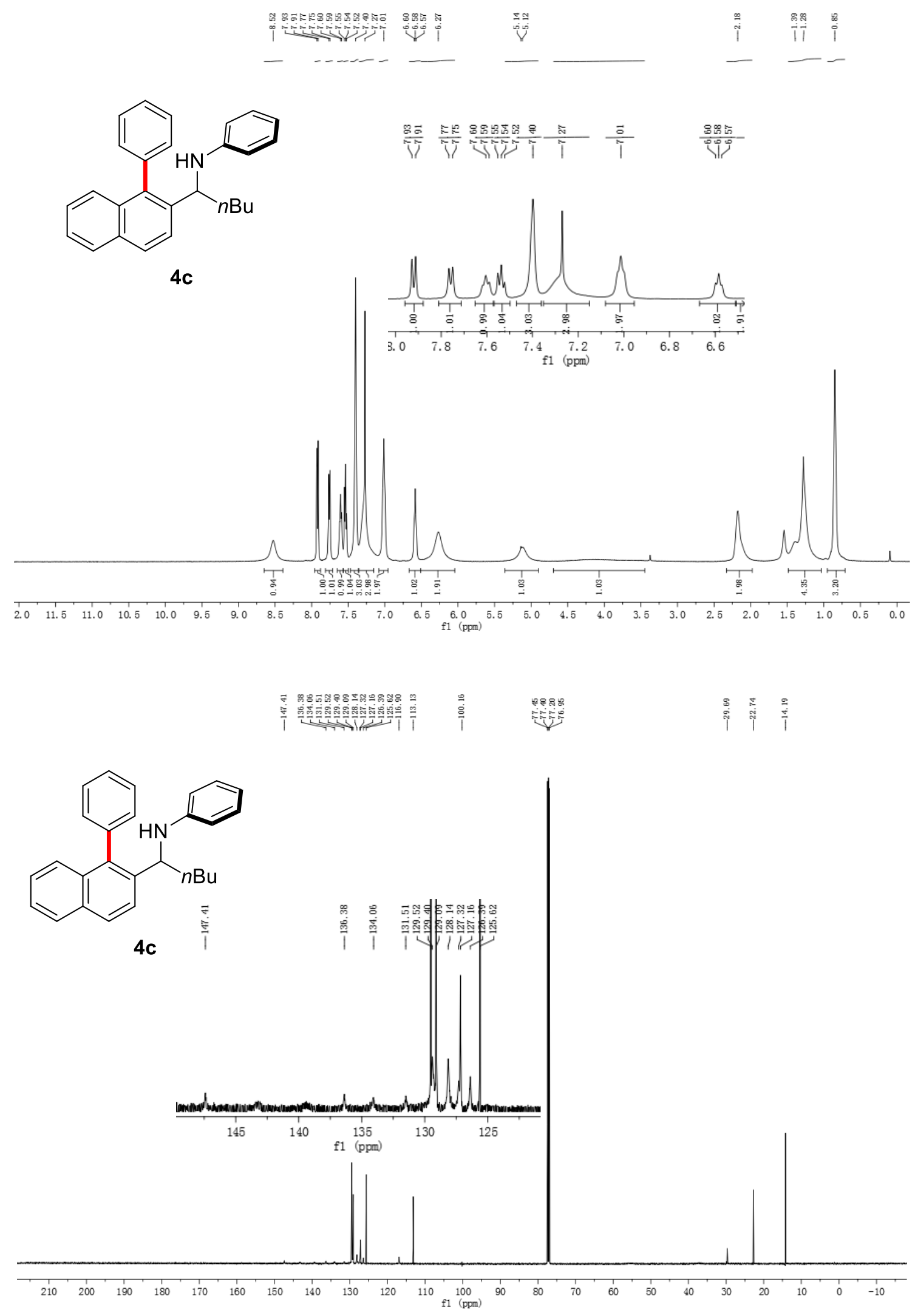




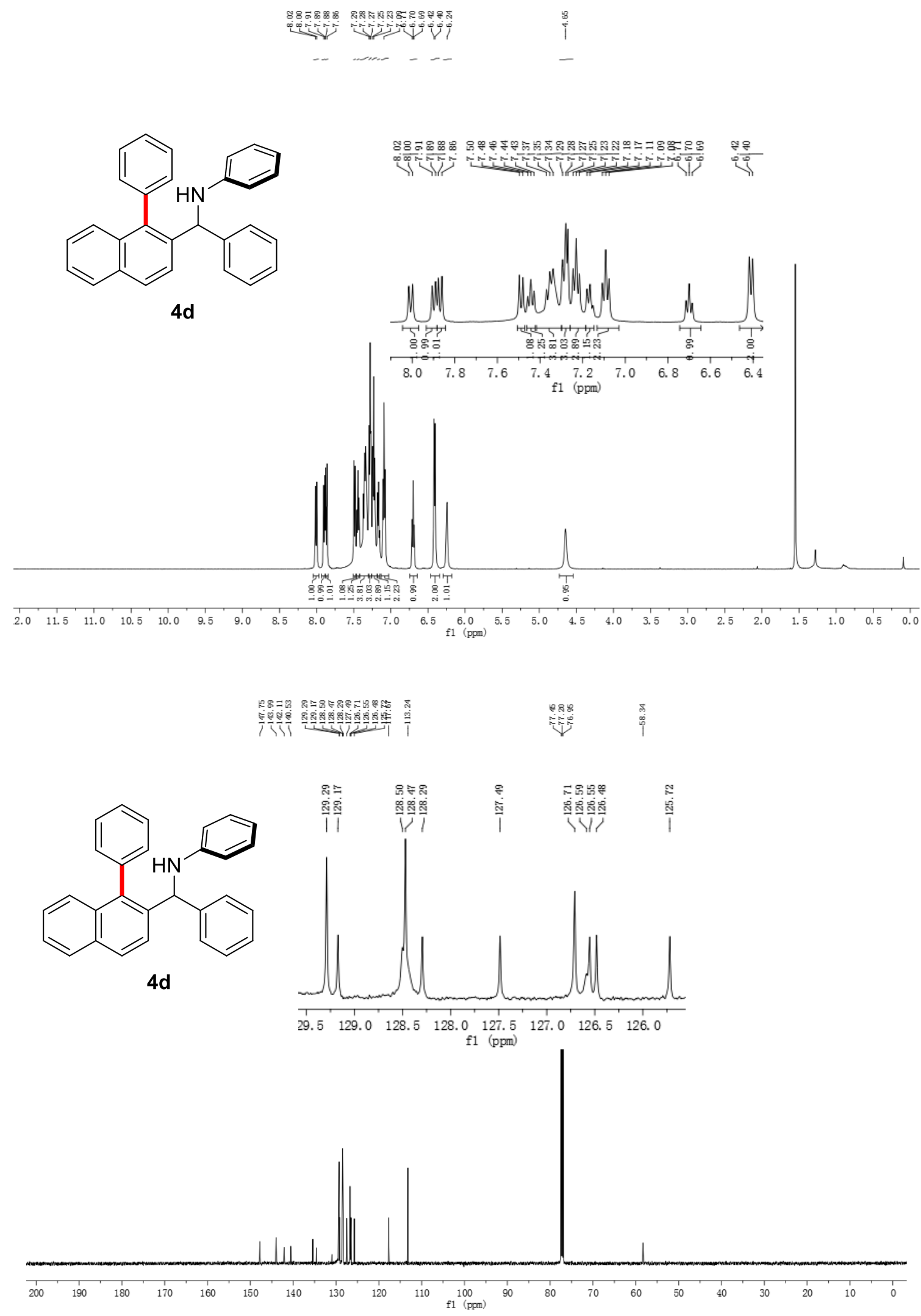




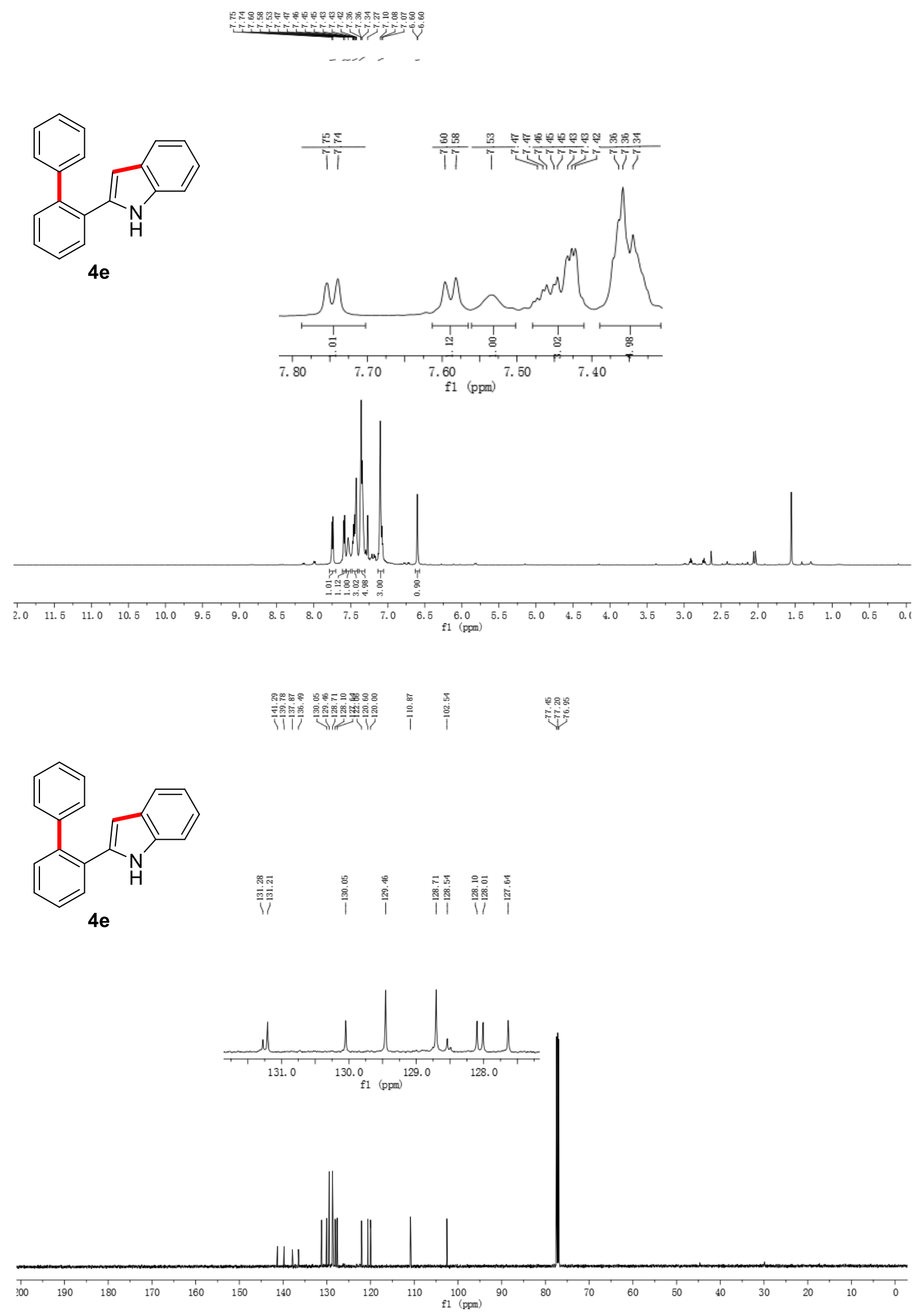

SI-77 